UDK 512.7

\title{
NEW MODULI COMPONENTS OF RANK 2 BUNDLES ON PROJECTIVE SPACE
}

\author{
CHARLES ALMEIDA, MARCOS JARDIM, ALEXANDER TIKHOMIROV, AND SERGEY TIKHOMIROV
}

\begin{abstract}
We present a new family of monads whose cohomology is a stable rank two vector bundle on $\mathbb{P}^{3}$. We also study the irreducibility and smoothness together with a geometrical description of some of these families. These facts are used to construct a new infinite series of rational moduli components of stable rank two vector bundles with trivial determinant and growing second Chern class. We also prove that the moduli space of stable rank two vector bundles with trivial determinant and second Chern class equal to 5 has exactly three irreducible rational components.
\end{abstract}

2010 MSC: 14D20, 14J60

Keywords: Rank 2 bundles, Monads, Instanton bundles

\section{INTRODUCTION}

In 33 Maruyama proved that the rank $r$ stable vector bundles on a projective scheme $X$ with fixed Chern classes $c_{1}, \ldots, c_{r}$ can be parametrized by an algebraic quasi-projective variety, denoted by $\mathcal{B}_{X}\left(r, c_{1}, \ldots, c_{r}\right)$. Although this result has been known for almost 40 years, there are just a few concrete examples and established facts about such varieties, even for cases like $X=\mathbb{P}^{3}$ and $r=2$. For instance, $\mathcal{B}_{\mathbb{P}^{3}}(2,0,1)$ was studied by Barth in $\left[3, \mathcal{B}_{\mathbb{P}^{3}}(2,0,2)\right.$ was described by Harthorne in [19, $\mathcal{B}_{\mathbb{P} 3}(2,-1,2)$ studied by Harthorne and Sols in [22] and by Manolache in 32 , while $\mathcal{B}_{\mathbb{P}^{3}}(2,-1,4)$ was described by Banica and Manolache in 1 . This probably happened due to the fact that the questions of irreducibility (solved in [36 and [37), and smoothness (answered in 28]) of the so-called instanton component of the moduli space $\mathcal{B}_{\mathbb{P}^{3}}\left(2,0, c_{2}\right)$ for all $c_{2} \in \mathbb{Z}_{+}$remained open until 2014.

In this paper, we continue the study of the moduli space $\mathcal{B}_{\mathbb{P}^{3}}(2,0, n)$, which we will simply denote by $\mathcal{B}(n)$ from now on, with the goal of providing new examples of families of vector bundles, and understanding their geometry. It is more or less clear from the table in [21, Section 5.3] that $\mathcal{B}(1)$ and $\mathcal{B}(2)$ should be irreducible, while $\mathcal{B}(3)$ and $\mathcal{B}(4)$ should have exactly two irreducible components; see [16] and [11, respectively, for the proof of the statements about $\mathcal{B}(3)$ and $\mathcal{B}(4)$. As for $\mathcal{B}(5)$, a description of all its irreducible components had been a challenge since 1980ies. In the paper, we give a complete answer to this problem - see Main Theorem 2 ,

For $n \geq 5$, two families of irreducible components have been studied, namely the instanton components, whose generic point corresponds to an instanton bundle, and the Ein components, whose generic point corresponds to a bundle given as cohomology of a monad of the form

$$
0 \rightarrow \mathcal{O}_{\mathbb{P}^{3}}(-c) \rightarrow \mathcal{O}_{\mathbb{P}^{3}}(-b) \oplus \mathcal{O}_{\mathbb{P}^{3}}(-a) \oplus \mathcal{O}_{\mathbb{P}^{3}}(a) \oplus \mathcal{O}_{\mathbb{P}^{3}}(b) \rightarrow \mathcal{O}_{\mathbb{P}^{3}}(c) \rightarrow 0
$$

where $b \geq a \geq 0$ and $c>a+b$. All of the components of $\mathcal{B}(n)$ for $n \leq 4$ are of either of these types; here we focus on a new family of bundles that appear as soon as $n \geq 5$.

More precisely, we study the set of vector bundles in $\mathcal{B}\left(a^{2}+k\right)$ for each $a \geq 2$ and $k \geq 1$ which arise as cohomologies of monads of the form:

$$
0 \rightarrow \mathcal{O}_{\mathbb{P}^{3}}(-a) \oplus V_{k} \otimes \mathcal{O}_{\mathbb{P}^{3}}(-1) \rightarrow V_{2 k+4} \otimes \mathcal{O}_{\mathbb{P}^{3}} \rightarrow V_{k}^{\prime} \otimes \mathcal{O}_{\mathbb{P}^{3}}(1) \oplus \mathcal{O}_{\mathbb{P}^{3}}(a) \rightarrow 0
$$

which will be denoted by $\mathcal{G}(a, k)$. We provide a bijection between such monads and monads of the form:

$$
0 \rightarrow \mathcal{O}_{\mathbb{P}^{3}}(-a) \stackrel{\sigma}{\rightarrow} \tilde{E} \stackrel{\tau}{\rightarrow} \mathcal{O}_{\mathbb{P}^{3}}(a) \rightarrow 0
$$

where $\tilde{E}$ is a symplectic rank 4 instanton bundle of charge $k$. When $k=1$, these facts are used to prove our first main result. (See Theorem 20 below.)

Main Theorem 1. For each $a \geq 2$ not equal to $3, \mathcal{G}(a, 1)$ is a nonsingular dense subset of a rational irreducible component of $\mathcal{B}\left(a^{2}+1\right)$ of dimension

$$
4 \cdot\left(\begin{array}{c}
a+3 \\
3
\end{array}\right)-a-1
$$

Our second main result provides a complete description of all the irreducible components of $\mathcal{B}(5)$. 
Main Theorem 2. The moduli space $\mathcal{B}(5)$ has exactly 3 rational irreducible components, namely: the instanton component, of dimension 37, which is nonsingular and consists of those bundles given as cohomology of monads of the form

$$
\begin{gathered}
0 \rightarrow V_{5} \otimes \mathcal{O}_{\mathbb{P}^{3}}(-1) \rightarrow V_{12} \otimes \mathcal{O}_{\mathbb{P}^{3}} \rightarrow V_{5} \otimes \mathcal{O}_{\mathbb{P}^{3}}(1) \rightarrow 0, \text { and } \\
0 \rightarrow V_{2} \otimes \mathcal{O}_{\mathbb{P}^{3}}(-2) \rightarrow V_{3} \otimes \mathcal{O}_{\mathbb{P}^{3}}(-1) \oplus V_{3} \otimes \mathcal{O}_{\mathbb{P}^{3}}(1) \rightarrow V_{2} \otimes \mathcal{O}_{\mathbb{P}^{3}}(2) \rightarrow 0 ;
\end{gathered}
$$

the Ein component, nonsingular of dimension 40, which consists of those bundles given as cohomology of monads of the form

$$
0 \rightarrow \mathcal{O}_{\mathbb{P}^{3}}(-3) \rightarrow \mathcal{O}_{\mathbb{P}^{3}}(-2) \oplus V_{2} \otimes \mathcal{O}_{\mathbb{P}^{3}} \oplus \mathcal{O}_{\mathbb{P}^{3}}(2) \rightarrow \mathcal{O}_{\mathbb{P}^{3}}(3) \rightarrow 0 ;
$$

the closure of the set $\mathcal{G}(2,1)$, of dimension 37 , which consists of those bundles given as cohomology of monads of the form

$$
0 \rightarrow \mathcal{O}_{\mathbb{P}^{3}}(-2) \oplus \mathcal{O}_{\mathbb{P}^{3}}(-1) \rightarrow V_{6} \otimes \mathcal{O}_{\mathbb{P}^{3}} \rightarrow \mathcal{O}_{\mathbb{P}^{3}}(1) \oplus \mathcal{O}_{\mathbb{P}^{3}}(2) \rightarrow 0
$$

and

$$
0 \rightarrow \mathcal{O}_{\mathbb{P}^{3}}(-2) \oplus V_{2} \otimes \mathcal{O}_{\mathbb{P}^{3}}(-1) \rightarrow \mathcal{O}_{\mathbb{P}^{3}}(-1) \oplus V_{6} \otimes \mathcal{O}_{\mathbb{P}^{3}} \oplus \mathcal{O}_{\mathbb{P}^{3}}(1) \rightarrow V_{2}^{\prime} \otimes \mathcal{O}_{\mathbb{P}^{3}}(1) \oplus \mathcal{O}_{\mathbb{P}^{3}}(2) \rightarrow 0 .
$$

Hartshorne and Rao proved in 21 that every stable rank 2 bundle $\mathcal{E}$ on $\mathbb{P}^{3}$ with Chern classes $c_{1}(\mathcal{E})=0$ and $c_{2}(\mathcal{E})=5$ is the cohomology of one of the monads listed above. Rao showed in 35] that bundles given as cohomology of monads of the form (3) lie in the closure of the family of instanton bundles of charge 5 , which was shown to be irreducible firstly by Coanda, Tikhomirov and Trautmann in [13]; see also [36. The irreducibility of the family of bundles which arise as cohomology of monads of the form (4) was established by Ein in [15.

The fact that the closure of $\mathcal{G}(2,1)$ is an irreducible rational component of $\mathcal{B}(5)$ is the particular case $a=2$ of Main Theorem 1. Finally, we show that the set of bundles given by the monads of the form (6) lies in the closure of $\mathcal{G}(2,1)$.

We now give a breaf sketch of the contents of the paper. In Section 2 we recall some general properties of monads and of symplectic instanton bundles on $\mathbb{P}^{3}$. We especially treat the rank 4 symplectic instantons of charge 1. Any such bundle $E$ is described as a middle term of an exact triple with a rank 2 trivial bundle at the left hand and a null correlation rank 2 sheaf at the right hand. In Section 3 we study the set $\mathcal{G}(a, k)$ of (the isomorphism classes of) the so-called modified instanton bundles which are rank 2 bundles that arise as cohomology bundles of monads of the form (11) with $a \geq 2$ and $k \geq 1$. We show that each modified instanton appears as cohomology bundle of a monad of the form

$$
0 \rightarrow \mathcal{O}_{\mathbb{P}^{3}}(-a) \rightarrow E \rightarrow \mathcal{O}_{\mathbb{P}^{3}}(a) \rightarrow 0
$$

where $E$ is a rank 4 symplectic instanton of charge $k$. In the case $k=1$, this relation will be essential for the further constructions.

In Section 4 we study the set $\mathcal{G}(a, 1)$. We construct three families of symplectic monads of the form (77). The first one is the universal family, with the base scheme $S$, of monads with $E$ splitting as $E=\mathcal{O}_{\mathbb{P}^{3}}^{\oplus 2} \oplus N$ where $N$ is a null correlation bundle. The second is a family, with the base scheme $\widetilde{S}$ containing $S$ as a dense open subset, of monads $E$ a general symplectic rank 4 instanton of charge 1 . The third is a family of monads with $E$ splitting as in the first one, but with a new base $Y$. All the three families inherit universal cohomology sheaves, and it is shown that the images of their corresponding modular morphisms to $\mathcal{B}\left(a^{2}+1\right)$ have the same closure $\overline{\mathcal{G}(a, 1)}$ - see Propositions 14 and 15. In Section 5 the three families mentioned above are used to prove the Main Theorem 1 see Theorem 20.

Sections 6 and 7 are devoted to the study of the monads of the form (6). In Section 6 we show that the cohomology sheaves $\mathcal{E}$ of those among such monads that are not reduced to the monads of the form (5) are closely related (by two subsequent elementary transformations - see Proposition 25) to rank 2 reflexive sheaves with Chern classes $(0,2,2 k), 0 \leq k \leq 3$. A complete classification of the moduli components of these reflexive sheaves performed in Section 7 - see Propositions 26 and 27- leads to the dimension estimate, given in Theorem 21] for the subset of the bundles $\mathcal{E}$ specified above. It follows that this subset is not a component of $\mathcal{B}(5)$, and we use this in Section 8 to prove the Main Theorem 2.

Acknowledgements. CA was supported by the FAPESP grant number 2014/08306-4, 2016/14376-0 and PNPD post-doctoral grant by the IME-USP from the University of São Paulo. This work was partially done during a visit to the University of Barcelona, and he is grateful for its hospitality. MJ is partially supported by the CNPq grant number 302889/2018-3 and the FAPESP Thematic Project number 2018/21391-1; this work was partially done during a visit to the University of Edinburgh with the support of the FAPESP grant number 2016/03759-6. AST worked on this article within the framework of the Academic Fund Program at HSE University 
in 2020-2021 (grant number 20-01-011) and within the framework of the Russian Academic Excellence Project "5-100". AST also acknowledges the support from the Max Planck Institute for Mathematics in Bonn, where part of this work was done during the winter of 2020.

Notation and Conventions.

- In this work, $\mathbf{k}$ is an algebraically closed field of characteristic zero.

- $V_{n}$, respectively, $U_{n}$ denotes a k-vector space of dimension $n$.

- $\langle v\rangle$ the 1-dimensional subspace of $V_{n}$ spanned by a nonzero vector $v \in V_{n}$.

- $\mathbf{P}(F):=\operatorname{Proj}\left(\operatorname{Sym}_{\mathcal{O}_{X}} F\right)$ the projective spectrum of $F$, for a coherent $\mathcal{O}_{X}$-sheaf $F$ on a given scheme $X$.

- $\mathcal{O}_{\mathbf{P}(F)}(1)$ the Grothendieck sheaf on $\mathbf{P}(F)$.

- $\mathbf{V}(F):=\operatorname{Spec}\left(\operatorname{Sym}_{\mathcal{O}_{X}} F\right)$, for $X$ and $F$ as above.

- $\mathbb{P}^{3}:=P\left(U_{4}\right)$ the projective 3 -space.

- $\operatorname{Isom}\left(V_{n} \otimes \mathcal{O}_{X}, F\right) \rightarrow X$ the principal $G L(n, \mathbf{k})$-bundle of frames of a rank $n$ locally free $\mathcal{O}_{X}$-sheaf $F$.

- $\mathbf{X}:=\mathbb{P}^{3} \times X$, for a given scheme $X$.

- $p_{X}: \mathbf{X} \rightarrow X$ the projection onto the second factor, for $\mathbf{X}$ and $X$ as above.

- $\mathbf{f}: \mathbf{X} \rightarrow \mathbf{Y}$ the morphism induced by the morphism of schemes $f: X \rightarrow Y$.

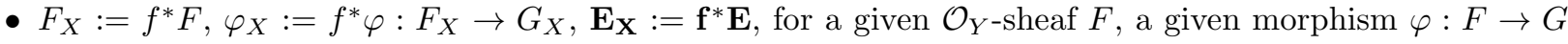
of $\mathcal{O}_{Y}$-sheaves, a given $\mathcal{O}_{\mathbf{Y}}$-sheaf (or, a complex of sheaves) $\mathbf{E}$, and $f: X \rightarrow Y$ and $\mathbf{f}: \mathbf{X} \rightarrow \mathbf{Y}$ as above.

- $\mathbf{E}(a, 0):=\mathbf{E} \otimes \mathcal{O}_{\mathbb{P}^{3}}(a) \otimes \mathcal{O}_{X}$, for $X$ and $\mathbf{E}$ as above, and $a \in \mathbb{Z}$.

- $X \stackrel{g_{X}}{\longleftarrow} X \times_{Z} Y \stackrel{f_{Y}}{\longrightarrow} Y$ the projections of the fibre product $X \times_{Z} Y$ induced by the morphisms $X \stackrel{f}{\rightarrow} Z \stackrel{g}{\leftarrow} Y$.

- $H^{i}(F)$ the $i$-th cohomology group of the sheaf $F$ on $\mathbb{P}^{3}$.

- $G r\left(n, V_{k}\right)$ the grassmannian variety of $n$-dimensional subspaces of $V_{k}$.

- Variety means an integral (i. e., reduced and irreducible) scheme.

- Since we are working with rank 2 vector bundles on $\mathbb{P}^{3}$, and Gieseker stability is equivalent to $\mu$-stability, we will not make any distinction between these two concepts.

- We will not make any distinction between vector bundles and locally free sheaves.

- $[E]$ the isomorphism class of a given sheaf on $\mathbb{P}^{3}$; in case $\mathcal{E}$ is a rank 2 stable sheaf on $\mathbb{P}^{3},[\mathcal{E}]$ is also considered as a point in the moduli space $M$ of stable rank 2 sheaves on $\mathbb{P}^{3}$.

- $\Phi_{X}: X \rightarrow M, x \mapsto\left[\left.\mathbf{E}\right|_{\mathbb{P}^{3} \times\{x\}}\right]$ the morphism defined by the $\mathcal{O}_{\mathbf{X}}$-sheaf $\mathbf{E}$ which is a family of stable rank 2 vector bundles on $\mathbb{P}^{3}$ with base $X$, for $M$ as above. We call $\Phi_{X}$ the modular morphism defined by the family $\mathbf{E}$.

- $\mathcal{R}(e, n, m)$ the set of isomorphism classes of rank 2 reflexive sheaves on $\mathbb{P}^{3}$ with Chern classes $\left(c_{1}, c_{2}, c_{3}\right)=$ $(e, n, m)$.

- $\ell(Y):=h^{0}\left(\mathcal{O}_{Y}\right)$ the length of a 0-dimensional scheme $Y$.

- $\mathrm{H}_{*}^{1}(E)=\bigoplus_{i \in \mathbb{Z}} \mathrm{H}^{1}(E(i))$ the graded cohomology module over the graded $\operatorname{ring} \Gamma_{*}\left(\mathcal{O}_{\mathbb{P}^{3}}\right):=\bigoplus_{j \geq 0} \mathrm{H}^{0}\left(\mathcal{O}_{\mathbb{P}^{3}}(j)\right)$.

- $(s)_{0}:=\{x \in X \mid s(x)=0\}$ the scheme of zeroes of a section $s$ of a given vector bundle on a scheme $X$.

- $\operatorname{Sp}(\mathcal{E})$ the spectrum of a vector bundle $[\mathcal{E}] \in \mathcal{B}(5)$, i. e., the nondecreasing sequence of integers $\left(a_{1}, a_{2}, a_{3}, a_{4}, a_{5}\right)$ uniquely defined by $\mathcal{E}$ - see [5, [20, Section 7].

- All the commutative diagrams of sheaves below which do not contain monads are assumed to have exact rows and columns. In these diagrams, the arrows $F \longmapsto G$, resp., $F \rightarrow G$ are shortenings for $0 \rightarrow F \rightarrow G$, resp., $F \rightarrow G \rightarrow 0$.

\section{Monads AND SYMPLECTiC INSTANTON BUndLES}

Recall that a monad is a complex of vector bundles of the form:

$$
0 \longrightarrow A \stackrel{\alpha}{\longrightarrow} B \stackrel{\beta}{\longrightarrow} C \longrightarrow 0
$$

such that $\alpha$ is injective, and $\beta$ is surjective. We call the sheaf $E:=\operatorname{ker} \beta / \operatorname{im} \alpha$ the cohomology of the monad (8). When $\alpha$ is locally left invertible (i. e., it is a subbundle morphism), then $E$ is a vector bundle.

The notion of monad is important in the study of vector bundles on $\mathbb{P}^{3}$ because Horrocks proved in [23] that every vector bundle on $\mathbb{P}^{3}$ is cohomology of a monad of the form (8) with $A, B$ and $C$ being sums of line bundles.

For completeness, we include in this section some useful results about monads that will be required in this work. The following lemma gives a relation between isomorphism classes of monads and its cohomology vector bundles; a proof can be found in [34, Lemma 4.1.3].

Lemma 1. Let $E$ and $E^{\prime}$ be, respectively, cohomology of the following monads:

$$
M: \quad 0 \longrightarrow A \stackrel{a}{\longrightarrow} B \stackrel{b}{\longrightarrow} C \longrightarrow 0,
$$




$$
M^{\prime}: \quad 0 \longrightarrow A^{\prime} \stackrel{a^{\prime}}{\longrightarrow} B^{\prime} \stackrel{b^{\prime}}{\longrightarrow} C^{\prime} \longrightarrow 0 .
$$

If one has that $\operatorname{Hom}\left(B, A^{\prime}\right)=\operatorname{Hom}\left(C, B^{\prime}\right)=\operatorname{Ext}^{1}\left(C, A^{\prime}\right)=\operatorname{Ext}^{1}\left(B, A^{\prime}\right)=\operatorname{Ext}^{1}\left(C, B^{\prime}\right)=\operatorname{Ext}^{2}\left(C, A^{\prime}\right)=0$, then there exists a bijection between the set of all morphisms from $E$ to $E^{\prime}$ and the set of all morphisms of monads from (9) to (10).

The following important corollary will be used several times in what follows, and a proof can also be found in 34, Lemma 4.1.3, Corollary 2].

Corollary 2. Consider the monad $M$ and its dual monad $M^{\vee}$, where:

$$
M: \quad 0 \longrightarrow A \stackrel{a}{\longrightarrow} B \stackrel{b}{\longrightarrow} C \longrightarrow 0, \quad M^{\vee}: \quad 0 \longrightarrow C^{\vee} \stackrel{b^{\vee}}{\longrightarrow} B^{\vee} \stackrel{a^{\vee}}{\longrightarrow} A^{\vee} \longrightarrow 0 .
$$

If these monads satisfy the hypothesis of Lemma 1, and there exists an isomorphism $f: E \rightarrow E^{\vee}$ between its cohomology bundles such that $f^{\vee}=-f$, then there are isomorphisms $h: C \rightarrow A^{\vee}$, and $q: B \rightarrow B^{\vee}$, such that $q^{\vee}=-q$, and $h \circ b=a^{\vee} \circ q$.

Recall that every locally free sheaf $E$ on $\mathbb{P}^{3}$ is the cohomology of a monad of the form [23]:

$$
0 \rightarrow \oplus_{i=1}^{r} \mathcal{O}_{\mathbb{P}^{3}}\left(a_{i}\right) \rightarrow \oplus_{j=1}^{s} \mathcal{O}_{\mathbb{P}^{3}}\left(b_{j}\right) \rightarrow \oplus_{k=1}^{t} \mathcal{O}_{\mathbb{P}^{3}}\left(c_{k}\right) \rightarrow 0 .
$$

In this work we will be interested in rank 2 locally free sheaves with vanishing first Chern class. Under these conditions, we have $E^{\vee} \simeq E$, and this implies that $t=r, s=2 r+2$, and $\left\{a_{i}\right\}=\left\{-c_{k}\right\}$. In addition, the middle entry of the monad is also self dual, so that (11) reduces to

$$
0 \rightarrow \oplus_{i=1}^{r} \mathcal{O}_{\mathbb{P}^{3}}\left(a_{i}\right) \rightarrow \oplus_{j=1}^{r+1}\left(\mathcal{O}_{\mathbb{P}^{3}}\left(b_{j}\right) \oplus \mathcal{O}_{\mathbb{P}^{3}}\left(-b_{j}\right)\right) \rightarrow \oplus_{i=1}^{r} \mathcal{O}_{\mathbb{P}^{3}}\left(-a_{i}\right) \rightarrow 0 .
$$

Finally, recall also that $r$ coincides with the number of generators of $\mathrm{H}_{*}^{1}(E)=\bigoplus_{p \in \mathbb{Z}} \mathrm{H}^{1}(E(p))$ as a graded module over the ring of homogeneous polynomials in four variables, while $a_{i}$ are the degrees of these generators, cf. 27, Thm. 2.3].

Instanton bundles are a particularly important class of stable rank 2 vector bundles due to their many remarkable properties and applications in mathematical physics. Besides this, instanton bundles form the only known irreducible component of the moduli space $\mathcal{B}(c)$ for every $c \in \mathbb{N}$.

In the remaining part of this section we will present the main results concerning instanton sheaves that will be used below. We start by recalling the definition of instanton sheaves on $\mathbb{P}^{3}$; see [25, Introduction] for further information on these objects.

Definition 3. An instanton sheaf on $\mathbb{P}^{3}$ is a torsion free coherent sheaf $E$ with $c_{1}(E)=0$ satisfying the following cohomological conditions:

$$
h^{0}(E(-1))=h^{1}(E(-2))=h^{2}(E(-2))=h^{3}(E(-3))=0 .
$$

The integer $n:=c_{2}(E)$ is called the charge of $E$. When $E$ is locally free, we say that $E$ is an instanton bundle.

We remark that instanton bundles of rank $r>2$ and non locally free instanton sheaves of rank $r \geq 2$ on $\mathbb{P}^{3}$ are not $\mu$-semistable in general, and also the vanishing of $h^{1}(E(-2))$ does not imply the vanishing of $h^{2}(E(-2))$. The definition above is the right generalization of the usual definition of an instanton vector bundle in the sense that, applying the Beilinson spectral sequence [34, Ch. II, Thm. 3.1.4]

$$
\mathrm{E}_{1}^{p q}=H^{q}\left(E(-p-1) \otimes \Omega_{\mathbb{P}^{3}}^{-p}\right) \otimes \mathcal{O}_{\mathbb{P}^{3}}(p+1) \Rightarrow \mathrm{E}_{\infty}^{p+q}= \begin{cases}E, & p+q=0 \\ 0, & p+q \neq 0\end{cases}
$$

to an arbitrary rank $r$ instanton sheaf $E$ of charge $k$, the vanishing (12) yields that $E$ is the cohomology of a monad of the form

$$
0 \rightarrow V_{k} \otimes \mathcal{O}_{\mathbb{P}^{3}}(-1) \rightarrow V_{r+2 k} \otimes \mathcal{O}_{\mathbb{P}^{3}} \rightarrow V_{k}^{\prime} \otimes \mathcal{O}_{\mathbb{P}^{3}}(1) \rightarrow 0 .
$$

Note that, conversely, the cohomology of a monad as above is an instanton sheaf as defined in Definition 3 , see [25, Thm. 3].

The cokernel $N$ of any monomorphism of sheaves $\mathcal{O}_{\mathbb{P}^{3}}(-1) \rightarrow \Omega_{\mathbb{P}^{3}}^{1}(1)$ is called a null correlation sheaf:

$$
0 \rightarrow \mathcal{O}_{\mathbb{P}^{3}}(-1) \stackrel{s}{\rightarrow} \Omega_{\mathbb{P}^{3}}^{1}(1) \rightarrow N \rightarrow 0 .
$$

Such sheaves are precisely the rank 2 instanton sheaves of charge 1, and are parametrized by the projective space $\mathbb{P} H^{0}\left(\Omega_{\mathbb{P}^{3}}^{1}(2)\right) \simeq \mathbb{P}^{5}$. If $N$ is locally free, we say that $N$ is a null correlation bundle. The set of non locally free null 
correlation sheaves are parametrized by the Grassmanian of lines in $\mathbb{P}^{3}$ : given a line $l \subset \mathbb{P}^{3}$ the corresponding null correlation sheaf $N_{l}$ is defined up to an isomorphism by the exact sequence

$$
0 \rightarrow N_{l} \rightarrow V_{2} \otimes \mathcal{O}_{\mathbb{P}^{3}} \stackrel{\varepsilon}{\rightarrow} \mathcal{O}_{l}(1) \rightarrow 0 .
$$

For the purposes of this paper, it is important to study rank 4 instanton bundles of charge 1 . Some of the following facts might be well known, but for lack of a reference we include proofs here.

Lemma 4. Every rank 4 instanton bundle $E$ of charge 1 over $\mathbb{P}^{3}$ fits into an exact sequence:

$$
0 \rightarrow V_{2} \otimes \mathcal{O}_{\mathbb{P}^{3}} \rightarrow E \rightarrow N \rightarrow 0,
$$

where $N$ is a null correlation sheaf. If $N$ is a null correlation bundle, then sequence (17) splits. In addition,

$$
h^{0}(E)=2, \quad h^{i}(E)=0, \quad i \geq 1 .
$$

Proof. As observed in the paragraph right below Definition 3, $E$ can be obtained as cohomology of a monad (14) for $r=4$ and $k=1$ :

$$
M_{E}: \quad 0 \longrightarrow \mathcal{O}_{\mathbb{P}^{3}}(-1) \stackrel{\alpha}{\longrightarrow} V_{6} \otimes \mathcal{O}_{\mathbb{P}^{3}} \stackrel{\beta}{\longrightarrow} \mathcal{O}_{\mathbb{P}^{3}}(1) \longrightarrow 0 .
$$

Without loss of generality, we can choose homogeneous coordinates $[x: y: z: w]$ in $\mathbb{P}^{3}$ and a basis in $V_{6}$, such that the map $\beta$ can be written as

$$
\beta:=\left(\begin{array}{llllll}
x & y & z & w & 0 & 0
\end{array}\right) .
$$

Hence using the display of the above monad, we have that $E$ fits into the following short exact sequence

$$
0 \rightarrow \mathcal{O}_{\mathbb{P}^{3}}(-1) \rightarrow V_{2} \otimes \mathcal{O}_{\mathbb{P}^{3}} \oplus \Omega(1) \rightarrow E \rightarrow 0 .
$$

From the above short exact sequence we can build up the following commutative diagramm

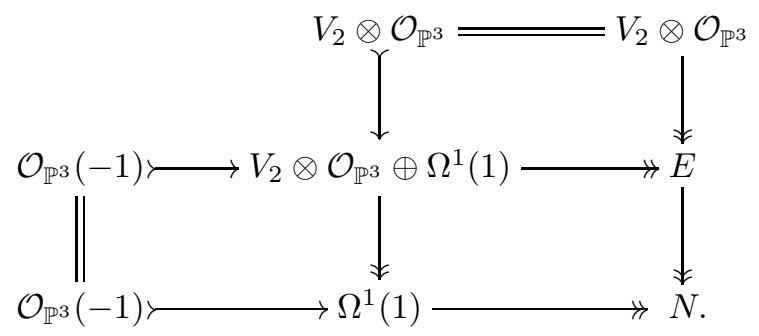

The rightmost column is the desired sequence.

If $N$ is locally free, then $\operatorname{Ext}^{1}\left(N, \mathcal{O}_{\mathbb{P}^{3}}\right) \simeq H^{1}(N)=0$, so the sequence in display (17) splits. The equality (18) follows from (17).

Remark. Assume that a bundle $E$ is the cohomology bundle of the monad (19). Then an easy cohomological computation shows that $E$ is a rank 4 instanton bundle of charge 1 .

Note that, substituting $N$ instead of $E$ into the Beilinson spectral sequence (13) yields the monad for $N$ :

$$
M_{N}: \quad 0 \rightarrow \mathcal{O}_{\mathbb{P}^{3}}(-1) \stackrel{\bar{\alpha}}{\rightarrow} V_{4} \otimes \mathcal{O}_{\mathbb{P}^{3}} \stackrel{\bar{\beta}}{\rightarrow} \mathcal{O}_{\mathbb{P}^{3}}(1) \rightarrow 0, \quad N=\operatorname{ker} \bar{\beta} / \operatorname{im} \bar{\alpha},
$$

fitting together with the monad (19) in the commutative diagram

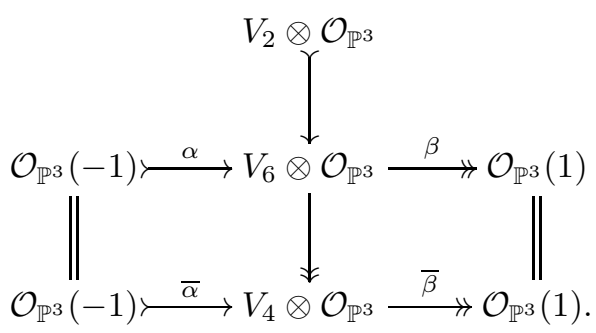

In this diagram the exact middle column is obtained from the exact triple $0 \rightarrow V_{2} \rightarrow V_{6} \rightarrow V_{4} \rightarrow 0$ arising as the cohomology sequence of the exact triple $0 \rightarrow V_{2} \otimes \Omega_{\mathbb{P}^{3}} \rightarrow E \otimes \Omega_{\mathbb{P}^{3}} \rightarrow N \otimes \Omega_{\mathbb{P}^{3}} \rightarrow 0$ induced by the triple (17). In addition, from (23) and (20) we obtain

$$
\bar{\beta}=\left(\begin{array}{llll}
x & y & z & w
\end{array}\right) .
$$


Proposition 5. Let $E$ be a rank 4 instanton bundle $E$ of charge 1 over $\mathbb{P}^{3}$, then $h^{0}\left(S^{2} E\right)=3, h^{1}\left(S^{2} E\right)=$ $5, h^{2}\left(S^{2} E\right)=0$.

Proof. Taking the symmetric power of the sequence in display (21), we obtain that $S^{2} E$ fits into the following short exact sequence:

$$
0 \longrightarrow V_{2} \otimes \mathcal{O}_{\mathbb{P}^{3}}(-1) \oplus \Omega \longrightarrow\left(S^{2} V_{2} \otimes \mathcal{O}_{\mathbb{P}^{3}}\right) \oplus\left(V_{2} \otimes \Omega(1)\right) \oplus S^{2} \Omega(2) \longrightarrow S^{2} E \longrightarrow
$$

From the long exact sequence of cohomology we have

$$
0 \rightarrow S^{2} V_{2} \rightarrow H^{0}\left(S^{2} E\right) \rightarrow \mathbf{k} \rightarrow \Lambda^{2} W^{\vee} \rightarrow H^{1}\left(S^{2} E\right) \rightarrow 0,
$$

where $W$ is the 4 -dimensional $\mathbf{k}$-vector space such that $\mathbb{P}^{3}=\mathbb{P}(W)$, and

$$
0 \rightarrow H^{2}\left(S^{2} E\right) \rightarrow 0 .
$$

From which we conclude that $H^{2}\left(S^{2} E\right)=0$. The map $\mathbf{k} \rightarrow \Lambda^{2} W^{\vee}$ is given by the skew-form corresponding to the morphism $\mathcal{O}_{\mathbb{P}^{3}}(-1) \rightarrow \Omega(1)$, in the definition of $E$, and in particular is non-zero, which implies that $\mathbf{k} \rightarrow \Lambda^{2} W^{\vee}$ is injective, and therefore

$$
H^{0}\left(S^{2} E\right) \simeq S^{2} V_{2} \text { and } H^{1}\left(S^{2} E\right) \simeq \Lambda^{2} W^{\vee} / \mathbf{k}
$$

from which our result follows.

In the remaining part of this section we will discuss the existence of a symplectic structure on an arbitrary rank 4 instanton bundle of charge 1 . Recall that a locally free sheaf $E$ is said to be symplectic if it admits a symplectic structure, that is, there exists an isomorphism $\varphi: E \rightarrow E^{\vee}$, such that $\varphi^{\vee}=-\varphi$. A symplectic instanton bundle is a pair $(E, \varphi)$ consisting of an instanton bundle $E$ together with a symplectic structure $\varphi$ on it; two symplectic instanton bundles $(E, \varphi)$ and $\left(E^{\prime}, \varphi^{\prime}\right)$ are isomorphic if there exists a bundle isomorphism $g: E \stackrel{\sim}{\rightarrow} E^{\prime}$ such that $\varphi=g^{\vee} \circ \varphi^{\prime} \circ g$.

Proposition 6. Any rank 4 instanton bundle $E$ of charge 1 admits a symplectic structure. In particular, if $E$ splits as $E=V_{2} \otimes \mathcal{O}_{\mathbb{P}^{3}} \oplus N$ where $N$ is a null correlation bundle, then any symplectic structure $\varphi$ on $E$ splits as $\varphi=\varphi_{1} \oplus \varphi_{2}$ where $\varphi_{1}$ and $\varphi_{2}$ are symplectic structures on $V_{2} \otimes \mathcal{O}_{\mathbb{P}^{3}}$ and $N$, respectively.

Proof. Let $E$ be an instanton rank 4 bundle. If $E$ splits as $E=V_{2} \otimes \mathcal{O}_{\mathbb{P} 3} \oplus N$, where $N$ is a null correlation bundle, then $\operatorname{det}\left(V_{2} \otimes \mathcal{O}_{\mathbb{P}^{3}}\right)=\operatorname{det} N=\mathcal{O}_{\mathbb{P}^{3}}$, hence both rank 2 bundles $V_{2} \otimes \mathcal{O}_{\mathbb{P}^{3}}$ and $N$ admit symplectic structures, say,

$$
\varphi_{1}: V_{2} \otimes \mathcal{O}_{\mathbb{P}^{3}} \stackrel{\simeq}{\rightarrow} V_{2}^{\vee} \otimes \mathcal{O}_{\mathbb{P}^{3}}, \quad \varphi_{2}: N \stackrel{\simeq}{\rightarrow} N^{\vee} \text {. }
$$

Then

$$
\varphi=\varphi_{1} \oplus \varphi_{2}: E \stackrel{\simeq}{\longrightarrow} E^{\vee}
$$

is a symplectic structure on $E$. Since

$$
\operatorname{Hom}\left(V_{2} \otimes \mathcal{O}_{\mathbb{P}^{3}}, N\right)=\operatorname{Hom}\left(N, V_{2} \otimes \mathcal{O}_{\mathbb{P}^{3}}\right)=0
$$

it follows immediately that any symplectic stucture on $E$ splits as in (26).

Note also that, in view of (15)

$$
\operatorname{Ext}^{i}\left(V_{2} \otimes \mathcal{O}_{\mathbb{P}^{3}}, N\right)=\operatorname{Ext}^{i}\left(N, V_{2} \otimes \mathcal{O}_{\mathbb{P}^{3}}\right)=0, \quad i \geq 1 .
$$

Now let $E$ be a non-splitting instanton, i. e. $E / V_{2} \otimes \mathcal{O}_{\mathbb{P}^{3}}$ is a null correlation sheaf $N_{l}$ which is not locally free at the points of the line $l$ given by the equations, say, $\{x=y=0\}$. This means that the morphism $\bar{\alpha}$ in the monad (22) for $N=N_{l}$ is vanishes at $l$, so that

$$
\bar{\alpha}=A\left(\begin{array}{l}
x \\
y
\end{array}\right), \quad A=\left(\alpha_{i j}\right), \quad 1 \leq i \leq 4,1 \leq j \leq 2,
$$

where $A$ is a $(4 \times 2)$-matrix of rank 2. The condition that $\bar{\beta} \circ \bar{\alpha}$ in (22) is the zero morphism together with (29) and (24) implies that all the coefficients $\alpha_{i j}$ of the matrix $A$, except $\alpha_{12}$ and $\alpha_{21}$, vanish and $\alpha_{12}+\alpha_{21}=0$. Thus, taking without loss of generality $\alpha_{12}=1$, we obtain

$$
\bar{\alpha}=\left(\begin{array}{c}
y \\
-x \\
0 \\
0
\end{array}\right)
$$


Since the cohomology sheaf of the middle monad in (23) is locally free, the morphism $\alpha$ in that diagram is a subbundle morphism. This together with (30) implies, again without loss of generality, that there exists a $(2 \times 2)$-matrix $C=\left(c_{i j}\right)$ such that

$$
\alpha=\left(\begin{array}{c}
y \\
-x \\
0 \\
0 \\
c_{11} x+c_{12} y+z \\
c_{21} x+c_{22} y+w
\end{array}\right) .
$$

It now follows from (31) and (20) that the skew-symmetric $(6 \times 6)$-matrix $J$ of the following $(2 \times 2)$-block form

$$
J=\left(\begin{array}{ccc}
Q & \mathbb{O} & -C^{t} \\
\mathbb{O} & \mathbb{O} & -\mathbb{1} \\
C & \mathbb{1} & \mathbb{O}
\end{array}\right), \quad \text { where } \quad Q=\left(\begin{array}{cc}
0 & -1 \\
1 & 0
\end{array}\right)
$$

satisfies the condition $\alpha=J \beta^{t}$. This means that, taking $-J$ for the matrix of the symplectic form $q: V_{6} \rightarrow V_{6}^{\vee}$ with respect to the above choice of the basis in $V_{6}$, we obtain that $\alpha$ and $\beta$ as morphisms satisfy the condition $\beta=\alpha^{\vee} \circ q$. In other words, the monad (19) is symplectic. Then by Corollary 2 its cohomology bundle $E$ also admits a symplectic structure.

\section{MODIFIED INSTANTON MONADS}

We will now study monads of the form (1), with $a \geq 2$ and $k \geq 1$ :

$$
0 \rightarrow \mathcal{O}_{\mathbb{P}^{3}}(-a) \oplus V_{k} \otimes \mathcal{O}_{\mathbb{P}^{3}}(-1) \stackrel{\alpha}{\rightarrow} V_{2 k+4} \otimes \mathcal{O}_{\mathbb{P}^{3}} \stackrel{\beta}{\rightarrow} \mathcal{O}_{\mathbb{P}^{3}}(a) \oplus V_{k}^{\prime} \otimes \mathcal{O}_{\mathbb{P}^{3}}(1) \rightarrow 0,
$$

which we call modified instanton monads. The set of isomorphism classes of bundles arising as cohomology of such monads will be denoted by $\mathcal{G}(a, k)$. Note that, so far, $\mathcal{G}(a, k)$ could possibly be empty.

Proposition 7. For each $a \geq 2$ and $k \geq 1$, the family $\mathcal{G}(a, k)$ is non-empty and contains stable bundles, while every $[\mathcal{E}] \in \mathcal{G}(a, k)$ is $\mu$-semistable. In addition, every $[\mathcal{E}] \in \mathcal{G}(a, 1)$ is stable.

Proof. Let $F$ be an rank 2 instanton bundle of charge $k$. Let $a \geq 2$ and take $\sigma \in \mathrm{H}^{0}(F(2 a))$ such that its zero locus $X:=(\sigma)_{0}$ is a curve; such $\sigma$ always exists if $F$ is a 't Hooft instanton bundle, for instance. Let $Y$ be a complete intersection curve given by the intersection of two surfaces of degree $a$ such that $X \cap Y=\emptyset$. According to [21, Lemma 4.8], there exists a bundle $E$ and a section $\tau \in \mathrm{H}^{0}(E(a))$ such that $(\tau)_{0}=Y \cup X$ which is given as cohomology of a monad of the form (32). In addition, since $F$ is stable, $X$ is not contained in any surface of degree $a$, hence neither is $Y \cup X$, and $\mathcal{E}$ is also stable.

It is straightforward to check that every $[\mathcal{E}] \in \mathcal{G}(a, k)$ satisfies $h^{0}(\mathcal{E}(-1))=0$, thus $\mathcal{E}$ is $\mu$-semistable.

Now fix $k=1$, and assume that there is $[\mathcal{E}] \in \mathcal{G}(a, 1)$ satisfying $h^{0}(\mathcal{E}) \neq 0$. Setting $K:=\operatorname{ker} \beta$, it follows that $h^{0}(K) \neq 0$, hence the quotient $K^{\prime}:=K / \mathcal{O}_{\mathbb{P}^{3}}$ fits into the following exact sequence

$$
0 \rightarrow K^{\prime} \rightarrow V_{5} \otimes \mathcal{O}_{\mathbb{P}^{3}} \stackrel{\beta^{\prime}}{\rightarrow} \mathcal{O}_{\mathbb{P}^{3}}(1) \oplus \mathcal{O}_{\mathbb{P}^{3}}(a) \rightarrow 0 .
$$

By [8, Thm. 2.7] $K^{\prime}$ is $\mu$-stable. However, the monomorphism $\alpha: \mathcal{O}_{\mathbb{P}^{3}}(-a) \oplus \mathcal{O}_{\mathbb{P}^{3}}(-1) \rightarrow K$ induces a monomorphism $\mathcal{O}_{\mathbb{P}^{3}}(-1) \rightarrow K^{\prime}$; by the $\mu$-stability of $K^{\prime}$, we should have

$$
-1<\mu\left(K^{\prime}\right)=-\frac{a+1}{3} \Longrightarrow a<2,
$$

providing the desired contradiction.

Remark. Note that the space $X$ of monads (32) is a locally closed subscheme of the affine space $A=$ $\operatorname{Hom}\left(\mathcal{O}_{\mathbb{P}^{3}}(-a) \oplus V_{k} \otimes \mathcal{O}_{\mathbb{P}^{3}}(-1), V_{2 k+4} \otimes \mathcal{O}_{\mathbb{P}^{3}}\right) \times \operatorname{Hom}\left(V_{2 k+4} \otimes \mathcal{O}_{\mathbb{P}^{3}}, \mathcal{O}_{\mathbb{P}^{3}}(a) \oplus V_{k}^{\prime} \otimes \mathcal{O}_{\mathbb{P}^{3}}(1)\right)$ defined as $X=\{(\alpha, \beta) \in$ $A \mid \alpha$ is a subbundle morphism, $\beta$ is an epimorphism and $\beta \circ \alpha=0\}$, and there is the universal cohomology bundle $\mathcal{E}$ on $\mathbf{X}$. In case $k=1$, it follows from Proposition 7 that $\mathcal{G}(a, 1)$ is the image of $X$ under the modular morphism $\Phi_{X}: X \rightarrow \mathcal{B}\left(a^{2}+1\right), x \mapsto\left[\left.\mathbf{E}\right|_{\mathbb{P}^{3} \times\{x\}}\right]$. Thus, $\mathcal{G}(a, 1)$ is a constructible set, i. e., a disjoint union of locally closed subsets of $\mathcal{B}\left(a^{2}+1\right)$.

Next, we provide a cohomological characterization for modified instanton bundles.

Proposition 8. A vector bundle $\mathcal{E}$ on $\mathbb{P}^{3}$ is the cohomology of a monad of the form (32) if and only if $\mathrm{H}_{*}^{1}(\mathcal{E})$ has one generator in degree $-a$ and $k$ generators in degree -1 , and its Chern classes are $c_{1}(\mathcal{E})=0$, and $c_{2}(\mathcal{E})=a^{2}+k$. 
Proof. The "only if" part is straightforward. If $\mathcal{E}$ is a self dual vector bundle on $\mathbb{P}^{3}$ with one generator in degree $-a$ and $k$ generators in degree -1 , then by [27, Thm. 2.3], $\mathcal{E}$ is cohomology of a monad of the type:

$$
0 \rightarrow \mathcal{O}_{\mathbb{P}^{3}}(-a) \oplus V_{k} \otimes \mathcal{O}_{\mathbb{P}^{3}}(-1) \stackrel{\alpha}{\rightarrow} \oplus_{i=1}^{2 k+4} \mathcal{O}_{\mathbb{P}^{3}}\left(k_{i}\right) \stackrel{\beta}{\rightarrow} \mathcal{O}_{\mathbb{P}^{3}}(a) \oplus V_{k} \otimes \mathcal{O}_{\mathbb{P}^{3}}(1) \rightarrow 0 .
$$

Computing the Chern class give us $c_{2}(\mathcal{E})=a^{2}+k-\sum_{i=1}^{6} k_{i}^{2}$, since $c_{2}(\mathcal{E})=a^{2}+k$, we have $k_{i}=0$ for all $i$.

The modified instanton bundles are also related to usual instanton bundles of higher rank in a very important way. The precise relationship is outlined in the next couple of lemmas, and then summarized in Proposition 12 below.

Lemma 9. (i) Given a vector bundle $[\mathcal{E}] \in \mathcal{G}(a, k)$, there exists a rank 4 instanton bundle $E$ of charge $k$, and sections $\sigma \in H^{0}(E(a)), \tau \in H^{0}\left(E^{\vee}(a)\right)$ such that the complex:

$$
0 \rightarrow \mathcal{O}_{\mathbb{P}^{3}}(-a) \stackrel{\sigma}{\rightarrow} E \stackrel{\tau}{\rightarrow} \mathcal{O}_{\mathbb{P}^{3}}(a) \rightarrow 0
$$

is a monad whose cohomology coincides with $\mathcal{E}$.

(ii) The construction of the monad (33) is functorial in the sense that, if $\mathcal{E} \stackrel{\sim}{\longrightarrow} \mathcal{E}^{\prime}$, then the induced isomorphism $E \stackrel{\sim}{\longrightarrow} E^{\prime}$ extends to an isomorphism of monads

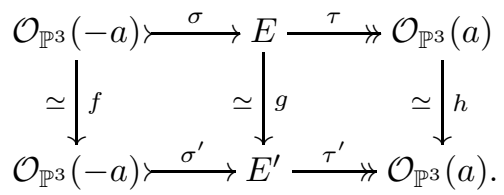

Proof. (i) Since $a \geq 2$, there is the canonical subbundle morphism $i: V_{k} \otimes \mathcal{O}_{\mathbb{P}^{3}}(-1) \rightarrow \mathcal{O}_{\mathbb{P}^{3}}(-a) \oplus V_{k} \otimes \mathcal{O}_{\mathbb{P}^{3}}(-1)$ which, together with the morphisms $\alpha$ and $\beta$ from the monad (32), yields a subbundle morphism $\alpha_{1}:=\alpha \circ i$ : $V_{k} \otimes \mathcal{O}_{\mathbb{P}^{3}}(-1) \rightarrow V_{2 k+4} \otimes \mathcal{O}_{\mathbb{P}^{3}}$ and an epimorphism $\beta_{1}:=i^{\vee} \circ \beta: V_{2 k+4} \otimes \mathcal{O}_{\mathbb{P}^{3}} \rightarrow V_{k}^{\prime} \otimes \mathcal{O}_{\mathbb{P}^{3}}(1)$. We thus obtain a new monad of type (14) with $r=4$ :

$$
0 \rightarrow V_{k} \otimes \mathcal{O}_{\mathbb{P}^{3}}(-1) \stackrel{\alpha_{1}}{\longrightarrow} V_{2 k+4} \otimes \mathcal{O}_{\mathbb{P}^{3}} \stackrel{\beta_{1}}{\rightarrow} V_{k}^{\prime} \otimes \mathcal{O}_{\mathbb{P}^{3}}(1) \rightarrow 0
$$

the cohomology bundle

$$
E=\frac{\operatorname{ker}\left(\beta_{1}\right)}{\operatorname{im}\left(\alpha_{1}\right)}
$$

of which is a rank-4 instanton, according to a remark after (14). The monads (32) and (35) fit in a commutative diagram with exact columns

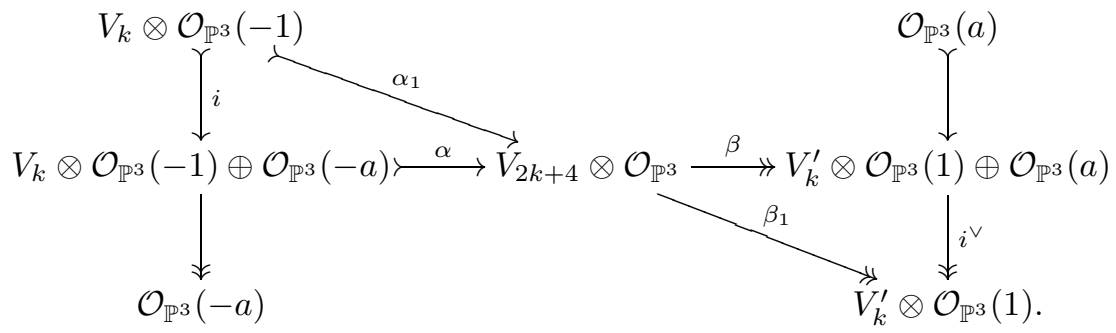

Now a standard diagram chasing with diagram (37) using (36) and the relation $\mathcal{E}=\frac{\operatorname{ker}(\beta)}{\operatorname{im}(\alpha)}$ yields a subbundle morphism $\mathcal{O}_{\mathbb{P}^{3}}(-a) \stackrel{\sigma}{\rightarrow} E$ and an epimorphism $E \stackrel{\tau}{\rightarrow} \mathcal{O}_{\mathbb{P}^{3}}(a)$ fitting in the monad (33) with the cohomology bundle $\mathcal{E}$.

(ii) Again, since $a \geq 2$, it follows immediately from (35) and (36) that $\operatorname{Hom}\left(\mathcal{O}_{\mathbb{P}^{3}}(a), E^{\prime}\right)=\operatorname{Hom}\left(E, \mathcal{O}_{\mathbb{P}^{3}}(-a)\right)=$ $\operatorname{Ext}^{1}\left(\mathcal{O}_{\mathbb{P}^{3}}(a), \mathcal{O}_{\mathbb{P}^{3}}(-a)\right)=\operatorname{Ext}^{1}\left(E, \mathcal{O}_{\mathbb{P}^{3}}(-a)\right)=\operatorname{Ext}^{1}\left(\mathcal{O}_{\mathbb{P}^{3}}(a), E^{\prime}\right)=\operatorname{Ext}^{2}\left(\mathcal{O}_{\mathbb{P}^{3}}(a), \mathcal{O}_{\mathbb{P}^{3}}(-a)\right)=0$ for the rank-4 instanton bundles $E$ and $E^{\prime}$ of charge $k$. The statement (ii) now follows from [34, Lemma 4.1.3].

Lemma 10. Given a monad (33) with E being a rank 4 instanton bundle of charge $k$, there is a monad of the form (32) whose cohomology coincides with the cohomology of the above monad.

Proof. This is a diagram chasing. Namely, by (14), $E$ is the cohomology of a monad of the form

$$
0 \rightarrow V_{k} \otimes \mathcal{O}_{\mathbb{P}^{3}}(-1) \stackrel{\alpha_{1}}{\longrightarrow} V_{2 k+4} \otimes \mathcal{O}_{\mathbb{P}^{3}} \stackrel{\beta_{1}}{\rightarrow} V_{k}^{\prime} \otimes \mathcal{O}_{\mathbb{P}^{3}}(1) \rightarrow 0 .
$$

This monad can be splitted to the exact triples of bundles

$$
0 \rightarrow E \rightarrow \operatorname{coker}\left(\alpha_{1}\right) \rightarrow V_{k}^{\prime} \otimes \mathcal{O}_{\mathbb{P}^{3}}(1) \rightarrow 0,
$$




$$
0 \rightarrow V_{k} \otimes \mathcal{O}_{\mathbb{P}^{3}}(-1) \stackrel{\alpha_{1}}{\longrightarrow} V_{2 k+4} \otimes \mathcal{O}_{\mathbb{P}^{3}} \stackrel{\varepsilon}{\rightarrow} \operatorname{coker}\left(\alpha_{1}\right) \rightarrow 0 .
$$

Respectively, the monad (33) splits into the exact triples

$$
0 \rightarrow \operatorname{ker}(\tau) \rightarrow E \stackrel{\tau}{\rightarrow} \mathcal{O}_{\mathbb{P}^{3}}(a) \rightarrow 0, \quad 0 \rightarrow \mathcal{O}_{\mathbb{P}^{3}}(-a) \rightarrow \operatorname{ker}(\tau) \stackrel{\delta}{\rightarrow} \mathcal{E} \rightarrow 0,
$$

where $\mathcal{E}$ is the cohomology bundle of the monad (33). The triple (39) and the first triple (41), together with the vanishing of $\operatorname{Ext}^{1}\left(V_{k}^{\prime} \otimes \mathcal{O}_{\mathbb{P}^{3}}(1), \mathcal{O}_{\mathbb{P}^{3}}(a)\right)$, yields by push-out the exact triple $0 \rightarrow \operatorname{ker}(\tau) \rightarrow \operatorname{coker}\left(\alpha_{1}\right) \stackrel{\gamma}{\rightarrow}$ $V_{k}^{\prime} \otimes \mathcal{O}_{\mathbb{P}^{3}}(1) \oplus \mathcal{O}_{\mathbb{P}^{3}}(a) \rightarrow 0$ which, together with (40), yields a commutative diagram in which we set $K:=\operatorname{ker}(\gamma \circ \varepsilon)$ :

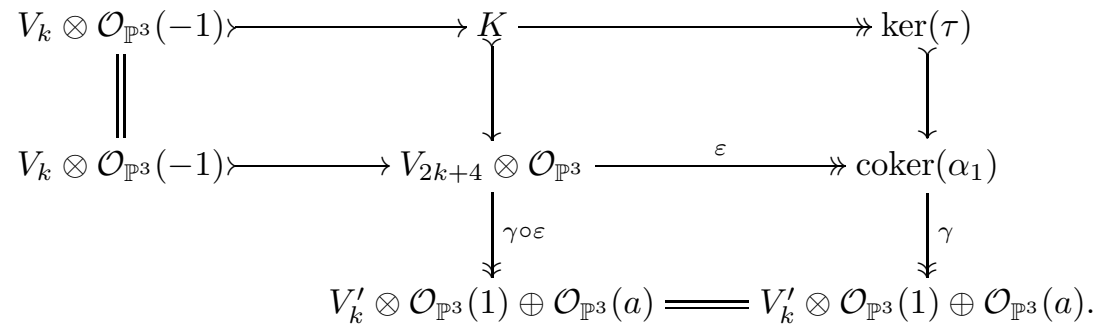

Similarly, the upper horizontal triple of this diagram, together with the second triple (41), yield the exact triple $0 \rightarrow V_{k} \otimes \mathcal{O}_{\mathbb{P}^{3}}(-1) \oplus \mathcal{O}_{\mathbb{P}^{3}}(-a) \rightarrow K \rightarrow \mathcal{E} \rightarrow 0$ which, being combined with the middle vertical triple in this diagram, yields the monad (32) with the cohomology bundle $\mathcal{E}$.

Next, we argue that the instanton bundle $E$ obtained in Lemma 9 comes with a natural symplectic structure.

Lemma 11. If $E$ is a rank 4 instanton bundle of charge $k$ that fits in a monad of the form (33), such that its cohomology sheaf $\mathcal{E}$ is a vector bundle, then $E$ admits a symplectic structure, and $\tau$ is determined by $\sigma$.

Proof. Since $\mathcal{E}$ is a rank 2 vector bundle with $c_{1}(\mathcal{E})=0$, there is a (unique up to scaling) symplectic isomorphism $\varphi: \mathcal{E} \cong \mathcal{E}^{\vee}$. Now, repeating the proof of Lemma 9 (ii) for $\mathcal{E}^{\prime}=\mathcal{E}^{\vee}$, we obtain an isomorphism of monads:

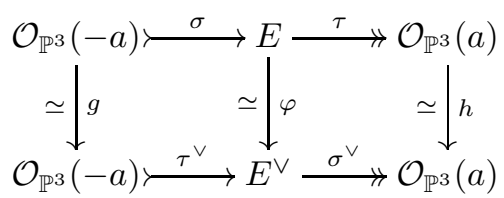

such that $\varphi^{\vee}=-\varphi$, so $(E, \varphi)$ is a symplectic instanton bundle, and $\tau=\sigma^{\vee} \circ \varphi$.

Putting Lemmas 9, 10 and 11 together, we obtain the following statement.

Proposition 12. A rank 2 bundle $\mathcal{E}$ belongs to $\mathcal{G}(a, k), i$. e., $\mathcal{E}$ is the cohomology of a monad of the form (32) if and only if it is also the cohomology $\mathcal{E}=\mathcal{H}^{0}\left(A_{E, \varphi, \sigma}\right)$ of a monad of the form:

$$
A_{E, \varphi, \sigma}: \quad 0 \rightarrow \mathcal{O}_{\mathbb{P}^{3}}(-a) \stackrel{\sigma}{\rightarrow} E \stackrel{\sigma^{\vee} \circ \varphi}{\longrightarrow} \mathcal{O}_{\mathbb{P}^{3}}(a) \rightarrow 0,
$$

where $(E, \varphi)$ is a rank 4 symplectic instanton bundle of charge $k$.

\section{Set $\mathcal{G}(a, 1)$ And Related families of Sheaves}

We introduce a piece of notation which we will use below. Denote by $\mathcal{I}(k)$ the set of isomorphism classes of symplectic rank 4 instanton bundles with $c_{2}=k$. As before, let $V_{k}$ and $V_{2 k+4}$ be the fixed vector spaces of dimensions $k$ and $2 k+4$, respectively, and let $\left(\wedge^{2} V_{2 k+4}^{\vee}\right)^{0}$ be an open subset of the vector space $\wedge^{2} V_{2 k+4}^{\vee}$ consisting of nondegenerate symplectic forms on $V_{2 k+4}$. Next, for a given morphism $\tilde{\alpha}: V_{k} \otimes \mathcal{O}_{\mathbb{P}^{3}}(-1) \rightarrow V_{2 k+4} \otimes \mathcal{O}_{\mathbb{P} 3}$ we denote by $a$ the homomorphism $V_{k} \otimes U_{4} \rightarrow V_{2 k+4}$ corresponding to the morphism $\tilde{\alpha}$ under the isomorphism $\operatorname{Hom}\left(V_{k} \otimes \mathcal{O}_{\mathbb{P}^{3}}(-1), V_{2 k+4} \otimes \mathcal{O}_{\mathbb{P}^{3}}\right) \cong W:=\operatorname{Hom}\left(V_{k} \otimes U_{4}, V_{2 k+4}\right)$, where $U_{4}:=H^{0}\left(\mathcal{O}_{\mathbb{P}^{3}}(1)\right)^{\vee}$. We will call $\tilde{\alpha}$ the morphism associated to $a \in W$.

Recall the description of symplectic rank 4 instantons $(E, \varphi)$ in terms of symplectic monads (43) below. Namely, for a given point

$$
m=(a, q) \in W \times\left(\wedge^{2} V_{2 k+4}^{\vee}\right)^{0}
$$

consider the monad (38) in which $\tilde{\alpha}$ the morphism associated to the homomorphism $a$, and the morphism $\tilde{\beta}$ is such that $\tilde{\beta}=\tilde{\alpha}^{t}(q)$, where $\tilde{\alpha}^{t}(q)$ is the composition $V_{2 k+4} \otimes \mathcal{O}_{\mathbb{P}^{3}} \stackrel{q \otimes \operatorname{id}_{\mathcal{O}^{3}}}{\longrightarrow} V_{2 k+4}^{\vee} \otimes \mathcal{O}_{\mathbb{P}^{3}} \stackrel{\tilde{\alpha}^{\vee}}{\longrightarrow} V_{k}^{\vee} \otimes \mathcal{O}_{\mathbb{P}^{3}}(1)$ :

$$
A_{m}: \quad 0 \rightarrow V_{k} \otimes \mathcal{O}_{\mathbb{P}^{3}}(-1) \stackrel{\tilde{\alpha}}{\rightarrow} V_{2 k+4} \otimes \mathcal{O}_{\mathbb{P}^{3}} \stackrel{\tilde{\alpha}^{t}(q)}{\longrightarrow} V_{k}^{\vee} \otimes \mathcal{O}_{\mathbb{P}^{3}}(1) \rightarrow 0 .
$$


We call $A_{m}$ a symplectic monad. We also will denote by $\mathcal{H}^{0}\left(A_{m}\right)$ the cohomology bundle of the monad $A_{m}$.

Consider the set $\mathcal{M}(k)$ of symplectic monads (43):

$$
\mathcal{M}(k)=\left\{(a, q) \in W \times\left(\wedge^{2} V_{2 k+4}^{\vee}\right)^{0} \mid(a, q) \text { satisfies the conditions (i)-(ii) }\right\}
$$

where:

(i) the morphism $\tilde{\alpha}$ associated to $a$ is a subbundle morphism,

(ii) the composition $\tilde{\alpha}^{t}(q) \circ \tilde{\alpha}$ is the zero morphism.

Since $W$ is a vector space, and the condition (i), resp., (ii) is an open, resp., closed condition on the point $a \in W$, it follows that $\mathcal{M}(k)$ has a natural structure of a locally closed subscheme of the affine space $W \times \wedge^{2} V_{2 k+4}^{\vee}$.

From now on we will restrict to the case $k=1$. Set $\widetilde{M}:=\mathcal{M}(1)$. Note that the condition (i) of the definition of $\mathcal{M}(k)$ is empty in the case $k=1$, since in this case the the vanishig of $\wedge^{2}\left(V_{1}^{\vee} \otimes \mathcal{O}_{\mathbb{P} 3}(1)\right)$ clearly implies $\alpha^{t}(q) \circ \alpha=0$. Hence, $\widetilde{M}$ is a nonempty open (hence dense) subset of the affine space $W \times \wedge^{2} V_{6}^{\vee}$, where $W=\operatorname{Hom}\left(V_{1} \otimes U_{4}, V_{6}\right) \simeq \mathbf{k}^{24}$. In particular, $\widetilde{M}$ is irreducible and

$$
\operatorname{dim} \widetilde{M}=\operatorname{dim} W+\operatorname{dim} \wedge^{2} V_{6}^{\vee}=45 .
$$

Proposition 13. Any rank 4 instanton of charge 1 appears as a cohomology bundle of a symplectic monad

$$
A_{m}: \quad 0 \rightarrow \mathcal{O}_{\mathbb{P}^{3}}(-1) \stackrel{\tilde{\alpha}}{\rightarrow} V_{6} \otimes \mathcal{O}_{\mathbb{P}^{3}} \stackrel{\tilde{\alpha}^{t}(q)}{\rightarrow} \mathcal{O}_{\mathbb{P}^{3}}(1) \rightarrow 0 .
$$

for some $m \in \widetilde{M}$.

Proof. Let $E$ be a rank 4 instanton of charge 1. According to Proposition (6), $E$ admits a symplectic structure $\varphi: E \stackrel{\sim}{\rightarrow} E^{\vee}$. It then known from [10, Section 3] that, under the condition $h^{0}(E)=h^{1}(-2)=0$ on a symplectic bundle $E$, this bundle is a cohomology of a symplectic monad from $\widetilde{M}$. However, the proof given therein, works without changes under the slightly weaker conditions (12) used in the Definition 3

On $\widetilde{\mathbf{M}}=\mathbb{P}^{3} \times \widetilde{M}$ there is the universal symplectic monad $\mathcal{A}_{\widetilde{\mathbf{M}}}: \quad 0 \rightarrow \mathcal{O}_{\widetilde{\mathbf{M}}}(-1,0) \stackrel{\boldsymbol{\alpha}}{\rightarrow} V_{6} \otimes \mathcal{O}_{\widetilde{\mathbf{M}}} \stackrel{\boldsymbol{\alpha}^{t}}{\rightarrow} \mathcal{O}_{\widetilde{\mathbf{M}}}(1,0) \rightarrow 0$ with the cohomology sheaf $\widetilde{\mathbf{E}}=\operatorname{ker} \boldsymbol{\alpha}^{t} / \operatorname{im} \boldsymbol{\alpha}$. Here $\boldsymbol{\alpha}^{t}=\boldsymbol{\alpha}^{\vee} \circ \mathbf{q}_{\widetilde{\mathbf{M}}}$ and $\mathbf{q}_{\widetilde{\mathbf{M}}}: V_{6} \otimes \mathcal{O}_{\widetilde{\mathbf{M}}} \stackrel{\sim}{\rightarrow} V_{6}^{\vee} \otimes \mathcal{O}_{\widetilde{\mathbf{M}}}$ is the tautological symplectic structure on $V_{6} \otimes \mathcal{O}_{\widetilde{\mathbf{M}}}$. From now on we fix an isomorphism of the monad $\mathcal{A}_{\widetilde{\mathbf{M}}}$ with its dual monad $\mathcal{A}_{\widetilde{\mathrm{M}}}^{\vee}$ by the following diagram:

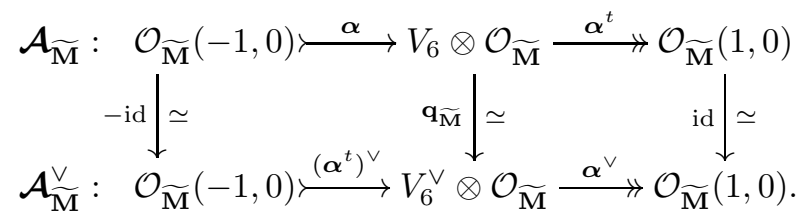

This isomorphism induces the symplectic structure

$$
\boldsymbol{\varphi}_{\widetilde{\mathbf{M}}}: \widetilde{\mathbf{E}} \stackrel{\simeq}{\rightarrow} \widetilde{\mathbf{E}}^{\vee}, \quad \text { and } \quad E_{m}=\left.\widetilde{\mathbf{E}}\right|_{\mathbb{P}^{3} \times\{m\}}, \quad \varphi_{m}=\left.\varphi_{\widetilde{\mathbf{M}}}\right|_{\mathbb{P}^{3} \times\{m\}}: E_{m} \stackrel{\sim}{\rightarrow} E_{m}^{\vee}, \quad m \in \widetilde{M},
$$

i. e. $\left(E_{m}, \varphi_{m}\right)$ is a symplectic rank 4 instanton of charge 1 . Note that, by the universality of the space $\widetilde{M}$, for any symplectic rank 4 instanton $(E, \varphi)$, there exists a unique point $m \in \widetilde{M}$ such that $(E, \varphi)=\left(E_{m}, \varphi_{m}\right)$, where $E_{m}$ and $\varphi_{m}$ are given by (47). It follows from (18) and the Base Change that the $\mathcal{O}_{\widetilde{M}}$-sheaf $\widetilde{\mathbf{U}}:=p_{\widetilde{M} *} \widetilde{\mathbf{E}}_{\text {is a rank }}$ 2 locally free sheaf and there is an exact triple on $\widetilde{\mathbf{M}}$, where ev is the canonical morphism:

$$
0 \rightarrow \widetilde{\mathbf{U}}_{\widetilde{\mathbf{M}}} \stackrel{\text { ev }}{\longrightarrow} \widetilde{\mathbf{E}} \rightarrow \widetilde{\mathbf{N}} \rightarrow 0, \quad \widetilde{\mathbf{N}}:=\operatorname{coker}(\mathbf{e v})
$$

and, for any $m \in \widetilde{M}$, the restriction of this triple onto $\mathbb{P}^{3} \times\{m\}$ coincides with the triple (17) for $E=E_{m}$. We thus have a map $\Psi: \widetilde{M} \rightarrow \mathbb{P}^{5}=P\left(\wedge^{2} V_{4}^{\vee}\right), m \mapsto\left[\left.\widetilde{\mathbf{N}}\right|_{\mathbb{P}^{3} \times\{m\}}\right]$. The map $\Psi$ has the following explicit description. Given a point $m=(a, q) \in \widetilde{M}$, consider a homomorphism $f(a, q): V_{4} \stackrel{a}{\rightarrow} V_{6} \stackrel{q}{\rightarrow} V_{6}^{\vee} \stackrel{a^{\vee}}{\rightarrow} V_{4}^{\vee}$. It is clearly skew- symmetric: $f(a, q) \in \wedge^{2} V_{4}^{\vee}$. An easy diagram chasing with the display of the monad $\left.\mathcal{A}_{\widetilde{\mathbf{M}}}\right|_{\mathbb{P}^{3} \times\{m\}}$ (i. e., equivalently, of the monad (46)) using (48) shows that

$$
\Psi(m)=\langle f(a, q)\rangle \in P\left(\wedge^{2} V_{4}^{\vee}\right),
$$

so that $\Psi$ is a well-defined morphism. By the universality of the monad $\mathcal{A}_{\widetilde{\mathbf{M}}}, \Psi$ is surjective.

We next consider the set

$$
M:=\left\{m \in \widetilde{M}|\widetilde{\mathbf{N}}|_{\mathbb{P}^{3} \times\{m\}} \text { is locally free }\right\}
$$


From the definition of $M$ it follows that it is a nonempty open subset of $\widetilde{M}$, hence it is irreducible since $\widetilde{M}$ is irreducible. Denote

$$
\mathbf{E}:=\widetilde{\mathbf{E}}_{\mathbf{M}}, \quad \varphi_{\mathbf{M}}:=\left(\varphi_{\widetilde{\mathbf{M}}}\right)_{\mathbf{M}}: \mathbf{E} \stackrel{\simeq}{\rightarrow} \mathbf{E}^{\vee}, \quad \mathbf{U}:=\widetilde{\mathbf{U}}_{M}, \quad \mathbf{N}:=\widetilde{\mathbf{N}}_{\mathbf{M}}
$$

where $\varphi_{\widetilde{\mathrm{M}}}$ is the symplectic structure (47). Note that, by Lemma 4 for any $m \in M$ the triple (48) restricted onto $\mathbb{P}^{3} \times\{m\}$ splits:

$$
E_{m} \simeq \mathcal{O}_{\mathbb{P}^{3}}^{\oplus 2} \oplus N_{m}, \quad m \in M,
$$

where $N_{m}$ is a null correlation bundle. We now show that these splittings globalize to the splitting of the triple $0 \rightarrow \mathbf{U} \rightarrow \mathbf{E} \rightarrow \mathbf{N} \rightarrow 0$ obtained from (48) by restriction onto $\mathbf{M}$ :

$$
\mathbf{E}=\mathbf{U} \oplus \mathbf{N} .
$$

Indeed, the last triple considered as an extension is given by the element in $\operatorname{Ext}^{1}(\mathbf{N}, \mathbf{U})$. By (27), (28) and the Base Change [31, Thm. 1.4], the sheaves $\mathcal{E} x t_{p_{M}}^{i}(\mathbf{N}, \mathbf{U}), i=0,1$, vanish, and the exact sequence relating global and relative Ext [31, (1)] yields $\operatorname{Ext}^{1}(\mathbf{N}, \mathbf{U})=0$.

Now, for $a \geq 2$ and any $m \in M$, the triple (17) twisted by $\mathcal{O}_{\mathbb{P}^{3}}(a)$, in which we set $E=E_{m}$, yields:

$$
h^{0}\left(E_{m}(a)\right)=4\left(\begin{array}{c}
a+3 \\
3
\end{array}\right)-a-2, \quad h^{i}\left(E_{m}(a)\right)=0, \quad i>0 .
$$

Formulas (47), (53) and the Base Change show that the sheaf

$$
F=p_{M *}(\mathbf{E}(a, 0))
$$

is a locally free $\mathcal{O}_{M}$-sheaf of rank $r=h^{0}\left(E_{m}(a)\right)$. Consider the scheme $T=\mathbf{P}\left(F^{\vee}\right)$. By the above, $T$ is set-theoretically described as

$$
T=\left\{(m,\langle\sigma\rangle) \mid m \in M, 0 \neq \sigma \in H^{0}\left(E_{m}(a)\right)\right\},
$$

and the natural projection $\rho: T \rightarrow M,(m,\langle\sigma\rangle) \mapsto m$ is a locally trivial $\mathbb{P}^{r-1}$-bundle. Note that, since $M$ is an open subset of the affine space $W$, it follows that $T$ is a variety, and from (45) and (53) we have

$$
\operatorname{dim} T=h^{0}\left(E_{m}(a)\right)-1+\operatorname{dim} M=4\left(\begin{array}{c}
a+3 \\
3
\end{array}\right)-a+42 .
$$

On $T$ and $\mathbf{M}$ we have canonical morphisms $F_{T}^{\vee} \stackrel{\text { ev }}{\rightarrow} L$ and $F_{\mathbf{M}} \stackrel{\text { can }}{\longrightarrow} \mathbf{E}(a, 0)$, respectively, where $L=\mathcal{O}_{\mathbf{P}\left(F^{\vee}\right)}(1)$ is the Grothendieck sheaf. Consider the composition of morphisms

$$
\boldsymbol{\sigma}: \mathcal{O}_{\mathbb{P}^{3}} \otimes L^{\vee} \stackrel{\mathrm{ev}_{\mathbf{T}}^{\vee}}{\longrightarrow} F_{\mathbf{T}} \stackrel{\mathrm{can}_{\mathbf{T}}}{\longrightarrow} \mathbf{E}_{\mathbf{T}}(a, 0) .
$$

By definition, for any point $(m, \mathbf{k} \sigma) \in T$ the restriction $\left.\boldsymbol{\sigma}\right|_{\mathbb{P}^{3} \times\{(m, \mathbf{k} \sigma)\}}$ coincides, up to a twist by $\mathcal{O}_{\mathbb{P}^{3}}(-a)$, with the morphism $\sigma: \mathcal{O}_{\mathbb{P}^{3}}(-a) \rightarrow E_{m}$. In view of (51) we may represent $\sigma$ as $\sigma=\left(\sigma_{1}, \sigma_{2}\right), \quad \sigma_{1} \in H^{0}\left(\mathcal{O}_{\mathbb{P}^{3}}^{\oplus 2}(a)\right), \quad \sigma_{2} \in$ $H^{0}\left(N_{m}(a)\right)$. For the pair $\sigma=\left(\sigma_{1}, \sigma_{2}\right) \neq(0,0)$ we will adopt in the sequel, together with the notation $\langle\sigma\rangle$, the following equivalent notation:

$$
\left[\sigma_{1}: \sigma_{2}\right]:=\langle\sigma\rangle=\left\{\left(\lambda \sigma_{1}, \lambda \sigma_{2}\right) \mid \lambda \in \mathbf{k}^{\times}\right\},
$$

and also understand $\left[\sigma_{1}: \sigma_{2}\right]$ as a point of the projective space $P\left(H^{0}\left(\mathcal{O}_{\mathbb{P}^{3}}^{\oplus 2}(a)\right) \oplus H^{0}\left(N_{m}(a)\right)\right)$. Under this notation, define an open subset $S$ of $T$ as

$$
\begin{aligned}
S:=\left\{\left(m,\left[\sigma_{1}: \sigma_{2}\right]\right) \in T \mid(i) \sigma=\left(\sigma_{1}, \sigma_{2}\right): \mathcal{O}_{\mathbb{P}^{3}}(-a) \rightarrow E_{m} \simeq \mathcal{O}_{\mathbb{P}^{3}}^{\oplus 2} \oplus N_{m}\right. \\
\left.\quad \text { is a subbundle morphism and }(i i) \sigma_{1}, \sigma_{2} \neq 0\right\} .
\end{aligned}
$$

The subset $S$ is clearly open in $T$. Moreover, it is nonempty. Indeed, for any point $m \in M, E_{m}$ decomposes as in (51). Take any $a \geq 2$. Since the direct summand $N_{m}$ is a null correlation bundle, it follows quickly from the triple (15) for $N=N_{m}$, twisted by $\mathcal{O}_{\mathbb{P}^{3}}(a)$, that $N_{m}(a)$ is generated by global sections. From this it follows easily (cf. [19. Proof of Prop. 1.4]) that a general section $\sigma_{2} \in H^{0}\left(N_{m}(a)\right)$ has 1-dimensional zero-locus $\left(\sigma_{2}\right)_{0}$. Next, since a general section $\sigma_{1} \in H^{0}\left(\mathcal{O}_{\mathbb{P}^{3}}^{\oplus 2}(a)\right)$ has for its zero locus a complete intersection curve $\left(\sigma_{1}\right)_{0}=D_{1} \cap D_{2}$ for two surfaces $D_{1}, D_{2}$ of degree $a$, it follows that for general $D_{1}$ and $D_{2}$ we have $\left(\sigma_{1}\right)_{0} \cap\left(\sigma_{2}\right)_{0}=\emptyset$. Hence, the section $\sigma=\left(\sigma_{1}, \sigma_{2}\right) \in H^{0}\left(E_{m}(a)\right)$ has no zeroes and therefore defines a subbundle morphism $\sigma: \mathcal{O}_{\mathbb{P}^{3}}(-a) \rightarrow E_{m}$.

It follows that $S$ is irreducible and dense in $T$ since $T$ is irreducible. The morphism $\sigma_{\mathbf{S}}$ is included in the $\operatorname{monad} \mathcal{A}:=\left(\mathcal{A}_{\widetilde{\mathbf{M}}}\right)_{\mathbf{S}}$ on $\mathbf{S}$ :

$$
\mathcal{A}: 0 \rightarrow \mathcal{O}_{\mathbb{P}^{3}}(-a) \otimes L^{\vee} \stackrel{\sigma_{\mathbf{S}}}{\longrightarrow} \mathbf{E}_{\mathbf{S}} \stackrel{\boldsymbol{\sigma}_{\mathbf{S}}^{t}}{\longrightarrow} \mathcal{O}_{\mathbb{P}^{3}}(a) \otimes L \rightarrow 0
$$


where $\boldsymbol{\sigma}_{\mathbf{S}}^{t}$ is the composition $\mathbf{E}_{\mathbf{S}} \stackrel{\varphi_{\mathbf{S}}}{\longrightarrow} \mathbf{E}_{\mathbf{S}}^{\vee} \stackrel{\sigma_{\mathbf{S}}^{\vee}}{\longrightarrow} \mathcal{O}_{\mathbb{P}^{3}}(a) \otimes L$. By construction, for any point $(m,\langle\sigma\rangle) \in S$, the restriction of the monad $\mathcal{A}$ onto $\mathbb{P}^{3} \times\{(m,\langle\sigma\rangle)\}$ is isomorphic to the monad $A_{E_{m}, \varphi_{m}, \sigma}$ in (42). Hence,

$$
\left.\mathcal{H}^{0}(\mathcal{A})\right|_{\mathbb{P}^{3} \times\{(m,\langle\sigma\rangle)\}}=\mathcal{H}^{0}\left(A_{E_{m}, \varphi_{m}, \sigma}\right), \quad(m,\langle\sigma\rangle) \in S .
$$

In (63)-(65) below we will extend the constructions (54)-(55), (59)-(61) of the data $F, T, S, \mathcal{A}$ and $\mathcal{H}^{0}(\mathcal{A})$ over $M$ to the constructions of the corresponding data $\widetilde{F}, \widetilde{T}, \widetilde{S}, \widetilde{\mathcal{A}}, \mathcal{H}^{0}(\widetilde{\mathcal{A}})$ over $\widetilde{M}$. As a consequence, it will follow:

$$
F=\widetilde{F}_{\mathbf{M}}, \quad T=M \times_{\widetilde{M}} \widetilde{T}, \quad S \stackrel{\text { open dense }}{\longrightarrow} \widetilde{S}, \quad \mathcal{A}=(\widetilde{\mathcal{A}})_{\mathbf{S}}, \quad \mathcal{H}^{0}(\mathcal{A})=\left(\mathcal{H}^{0}(\widetilde{\mathcal{A}})\right)_{\mathbf{S}}
$$

For this, we first set

$$
\widetilde{F}:=p_{\widetilde{M} *}(\widetilde{\mathbf{E}}(a, 0)), \quad \widetilde{T}:=\mathbf{P}\left(\widetilde{F}^{\vee}\right),
$$

and remark that formulas (53) are still true for any $m \in \widetilde{M}$, so that the sheaf $\widetilde{F}$ is a locally free $\mathcal{O}_{\widetilde{M}}$-sheaf of rank $r=h^{0}\left(E_{m}(a)\right)$ given by (53), and the scheme $\widetilde{T}:=\mathbf{P}\left(\widetilde{F}^{\vee}\right)$ is set-theoretically described as $\widetilde{T}=\{(m,\langle\sigma\rangle) \mid m \in$ $\left.\widetilde{\mathcal{M}}, 0 \neq \sigma \in H^{0}\left(E_{m}(a)\right)\right\}$. The natural projection $\widetilde{\rho}: \widetilde{T} \rightarrow \widetilde{M},(m,\langle\sigma\rangle) \mapsto m$ is a locally trivial $\mathbb{P}^{r-1}$-bundle, so that, since $\widetilde{M}$ is an open subset of the affine space $W$, it follows that $\widetilde{T}$ is an irreducible variety of dimension

$$
\operatorname{dim} \widetilde{T}=h^{0}\left(E_{m}(a)\right)-1+\operatorname{dim} \widetilde{M}=4\left(\begin{array}{c}
a+3 \\
3
\end{array}\right)-a+42 .
$$

Here, in accordance with (56), $\widetilde{T}$ and $T$ have the same dimension. Next, we have an open subset $\widetilde{S}$ of $\widetilde{T}$ defined as $\widetilde{S}:=\left\{(m,\langle\sigma\rangle) \in \widetilde{T} \mid \sigma: \mathcal{O}_{\mathbb{P}^{3}}(-a) \rightarrow E_{m}\right.$ is a subbundle morphism. $\}$ Since the condition (ii) in (59) is open, comparing the definition of $\widetilde{S}$ with (59) we obtain that $S$ is an open subset of $T \cap \widetilde{S}$, where the intersection is taken in $\widetilde{T}$. Since $S$ is nonempty and $\widetilde{T}$ is irreducible, the inclusion $S \stackrel{\text { open dense }}{\longrightarrow} \widetilde{S}$ in (62) follows and, moreover, $\tilde{\rho}_{S}: S \rightarrow M$ coincides with the projection $\rho$.

Next, we have the extension of the universal monad (60) from $\mathbf{S}$ to $\widetilde{\mathbf{S}}: \widetilde{\mathcal{A}}: \quad 0 \rightarrow \mathcal{O}_{\mathbb{P}^{3}}(-a) \otimes L^{\vee} \stackrel{\boldsymbol{\sigma}}{\rightarrow} \widetilde{\mathbf{E}}_{\widetilde{\mathbf{S}}} \stackrel{\boldsymbol{\sigma}^{t}}{\rightarrow}$ $\mathcal{O}_{\mathbb{P}^{3}}(a) \otimes L \rightarrow 0$, satisfying the relation similar to (61):

$$
\left.\mathcal{H}^{0}(\widetilde{\mathcal{A}})\right|_{\mathbb{P}^{3} \times\{(m,\langle\sigma\rangle)\}}=\mathcal{H}^{0}\left(A_{E_{m}, \varphi_{m}, \sigma}\right), \quad(m,\langle\sigma\rangle) \in \widetilde{S} .
$$

Whence, the relations (62) follow from (50), (63) and the Base Change.

Consider the modular morphisms

$$
\Phi_{S}: S \rightarrow \mathcal{B}\left(a^{2}+1\right), \quad \Phi_{\widetilde{S}}: \widetilde{S} \rightarrow \mathcal{B}\left(a^{2}+1\right),
$$

defined by the families of sheaves $\mathcal{H}^{0}(\mathcal{A})$ and $\mathcal{H}^{0}(\tilde{\mathcal{A}})$, respectively. The relations (62), (65), and Proposition 12 together with the irreducibility of $\widetilde{S}$ yield

Proposition 14. (i) For $a \geq 2$, the set $\mathcal{G}(a, 1)$ of isomorphism classes of cohomology sheaves of monads (32) for $k=1$ is the image of the modular morphism

$$
\Phi_{\widetilde{S}}: \widetilde{S} \rightarrow \mathcal{B}\left(a^{2}+1\right), \quad(m,\langle\sigma\rangle) \mapsto\left[\left.\mathcal{H}^{0}(\widetilde{\mathcal{A}})\right|_{\mathbb{P}^{3} \times\{(m,\langle\sigma\rangle)\}}\right],
$$

defined by the family $\mathcal{H}^{0}(\widetilde{\mathcal{A}})$ of sheaves over $\widetilde{S}$. Its closure $\overline{\mathcal{G}(a, 1)}$ in $\mathcal{B}\left(a^{2}+1\right)$ is an irreducible scheme.

(ii) The set $\mathcal{G}(a, 1)_{0}:=\Phi_{S}(S)$ is dense in $\overline{\mathcal{G}(a, 1)}$.

In the remaining part of this section we will construct a new family of monads $\mathbf{A}_{\mathbf{Y}}$ on $\mathbb{P}^{3}$, with base $Y$ and cohomology sheaves belonging to $\mathcal{G}(a, 1)$, for which the related modular morphism

$$
\Phi_{Y}: Y \rightarrow \mathcal{B}\left(a^{2}+1\right), \quad y \mapsto\left[\left.\mathcal{H}^{0}\left(\mathbf{A}_{\mathbf{Y}}\right)\right|_{\mathbb{P}^{3} \times\{y\}}\right]
$$

has $\mathcal{G}(a, 1)_{0}$ as its image (see Proposition 15 below). This family will be used in the next Section to prove one of the main results of the paper - the rationality of $\overline{\mathcal{G}(a, 1)}$.

To construct the variety $Y$, consider the moduli space of $B:=\mathcal{B}(1)$ of locally free null correlation bundles on $\mathbb{P}^{3}$. This is well known to be isomorphic to $\mathbb{P}^{5} \backslash G(2,4)$, where $G(2,4)$ is the Plücker hyperquadric (see, e.g., 34 , Thm. 4.3.4]). Moreover, on $\mathbf{B}=\mathbb{P}^{3} \times B$ there is the universal family $\mathcal{N}$ of null correlation bundles. Consider the vector bundle $\mathcal{E}=V_{2} \otimes \mathcal{O}_{\mathbf{B}} \oplus \mathcal{N}$ and denote $E_{b}=\left.\mathcal{E}\right|_{\mathbb{P}^{3} \times\{b\}}, N_{b}=\left.\mathcal{N}\right|_{\mathbb{P}^{3} \times\{b\}}, b \in B$, so that

$$
E_{b}=V_{2} \otimes \mathcal{O}_{\mathbb{P}^{3}} \oplus N_{b}, \quad b \in B .
$$

By linear algebra, there are canonical isomorphisms $\varphi_{(1)}: V_{2} \otimes \mathcal{O}_{\mathrm{B}} \stackrel{\simeq}{\rightarrow} V_{2}^{\vee} \otimes \wedge^{2} V_{2} \otimes \mathcal{O}_{\mathrm{B}}$ and $\varphi_{(2)}: \mathcal{\mathcal { N }} \stackrel{\simeq}{\rightarrow} \mathcal{N}^{\vee} \otimes \wedge^{2} \mathcal{N}$. The sheaf $\mathcal{N}$ fits in the exact triple $0 \rightarrow \mathcal{O}_{\mathbb{P}^{3}} \otimes \mathcal{O}_{B}(-1) \rightarrow \Omega_{\mathbb{P}^{3}}^{1}(1) \otimes \mathcal{O}_{B} \rightarrow \mathcal{N} \rightarrow 0$ globalizing (15), so that $\wedge^{2} \mathcal{N} \simeq \mathcal{O}_{\mathbb{P}^{3}} \otimes \mathcal{O}_{B}(1)$. (Here we set $\left.\mathcal{O}_{B}( \pm 1):=\left.\mathcal{O}_{G(2,4)}( \pm 1)\right|_{B}.\right)$ Consider the varieties $B_{1}:=\mathbf{V}\left(\wedge^{2} V_{2}^{\vee} \otimes \mathcal{O}_{B}\right) \backslash$ 
$\{0-$ section $\} \stackrel{\pi_{1}}{\longrightarrow} B$ and $B_{2}:=\mathbf{V}\left(\mathcal{O}_{B}(-1)\right) \backslash\{0-$ section $\} \stackrel{\pi_{2}}{\longrightarrow} B$. Note that the pullback of a line bundle onto its total space with the 0 -section removed trivializes this bundle, we obtain $\pi_{2}^{*} \mathcal{O}_{B}(1) \simeq \mathcal{O}_{B_{2}}$, hence $\left(\wedge^{2} \mathcal{N}\right)_{\mathbf{B}_{2}} \simeq \mathcal{O}_{\mathbf{B}_{2}}$. Similarly, $\left(\wedge^{2} V_{2} \otimes \mathcal{O}_{\mathbf{B}}\right)_{\mathbf{B}_{1}} \simeq \mathcal{O}_{\mathbf{B}_{1}}$. Thus, we obtain the symplectic structures

$$
\varphi_{\mathbf{B}_{1}}:=\left(\varphi_{(1)}\right)_{\mathbf{B}_{1}}: V_{2} \otimes \mathcal{O}_{\mathbf{B}_{1}} \stackrel{\simeq}{\rightarrow} V_{2}^{\vee} \otimes \mathcal{O}_{\mathbf{B}_{1}}, \quad \varphi_{\mathbf{B}_{2}}:=\left(\varphi_{(2)}\right)_{\mathbf{B}_{2}}: \mathcal{N}_{\mathbf{B}_{2}} \stackrel{\simeq}{\rightarrow} \mathcal{N}_{\mathbf{B}_{2}}^{\vee} .
$$

Consider the variety $\widetilde{B}:=B_{1} \times_{B} B_{2}$. On $\widetilde{\mathbf{B}}$ we obtain from $\mathcal{E}$ a vector bundle $\mathcal{E}_{\widetilde{\mathrm{B}}}$ with the symplectic structure $\varphi_{\widetilde{\mathrm{B}}}$, where

$$
\mathcal{E}_{\widetilde{\mathrm{B}}}=V_{2} \otimes \mathcal{O}_{\widetilde{\mathrm{B}}} \oplus \mathcal{N}_{\widetilde{\mathrm{B}}}, \quad \varphi_{\widetilde{\mathrm{B}}}=\varphi_{1} \oplus \varphi_{2}: \mathcal{E}_{\widetilde{\mathrm{B}}} \rightarrow \mathcal{E}_{\widetilde{\mathrm{B}}}^{\vee}
$$

and $\varphi_{1}:=\left(\varphi_{\mathbf{B}_{1}}\right)_{\widetilde{\mathbf{B}}}: V_{2} \otimes \mathcal{O}_{\widetilde{\mathrm{B}}} \stackrel{\simeq}{\rightarrow} V_{2}^{\vee} \otimes \mathcal{O}_{\widetilde{\mathbf{B}}}, \varphi_{2}:=\left(\varphi_{\mathbf{B}_{2}}\right)_{\widetilde{\mathbf{B}}}: \mathcal{N}_{\widetilde{\mathrm{B}}} \stackrel{\simeq}{\rightarrow} \mathcal{N}_{\widetilde{\mathbf{B}}}^{\vee}$. By the above, we have the following description of the varieties $B_{1}, B_{2}$ and $\widetilde{B}$ :

$$
\begin{aligned}
& B_{1}=\left\{\left(b, \varphi_{1}\right) \mid b \in B, \varphi_{1}: V_{2} \otimes \mathcal{O}_{\mathbb{P}^{3}} \stackrel{\simeq}{\rightarrow} V_{2}^{\vee} \otimes \mathcal{O}_{\mathbb{P}^{3}} \text { is a symplectic structure }\right\}, \\
& B_{2}=\left\{\left(b, \varphi_{2}\right) \mid b \in B, \varphi_{2}: N_{b} \stackrel{\simeq}{\rightarrow} N_{b}^{\vee} \text { is a symplectic structure }\right\}, \\
& \widetilde{B}=\left\{\left(b, \varphi_{1}, \varphi_{2}\right) \mid\left(b, \varphi_{i}\right) \in B_{i}, i=1,2\right\} .
\end{aligned}
$$

The following constructions (see (71)-(76)) are parallel to the constructions (59)-(61). Twisting the equality (67) by $\mathcal{O}_{\mathbb{P}}(a)$, we obtain as in (55): $h^{0}\left(E_{b}(a)\right)=4\left(\begin{array}{c}a+3 \\ 3\end{array}\right)-a-2, h^{i}\left(E_{b}(a)\right)=0, i>0$. Thus, as in (54), the sheaf $F_{B}=p_{B *}(\mathcal{E}(a, 0))$ is a locally free $\mathcal{O}_{B}$-sheaf of rank $r=h^{0}\left(E_{b}(a)\right)$. Consider the variety $\mathcal{T}:=\mathbf{P}\left(F_{B}^{\vee}\right)$. Similarly to (55) we have

$$
\mathcal{T}=\left\{(b,\langle\sigma\rangle) \mid b \in B, 0 \neq \sigma \in H^{0}\left(E_{b}(a)\right)\right\} .
$$

For any point $(b,\langle\sigma\rangle) \in \mathcal{T}$ in view of (67) we may represent $\sigma$ as a pair $\sigma=\left(\sigma_{1}, \sigma_{2}\right), \sigma_{1} \in H^{0}\left(V_{2} \otimes \mathcal{O}_{\mathbb{P}^{3}}(a)\right), \sigma_{2} \in$ $H^{0}\left(N_{b}(a)\right)$. Thus, using the notation (58) we can rewrite (70) as $\mathcal{T}=\left\{\left(b,\left[\sigma_{1}: \sigma_{2}\right]\right) \mid b \in B,\left[\sigma_{1}: \sigma_{2}\right] \in\right.$ $\left.P\left(H^{0}\left(E_{b}(a)\right)\right)\right\}$. On the other hand, representing $\sigma$ as a morphism $\sigma: \mathcal{O}_{\mathbb{P}^{3}}(-a) \rightarrow E_{b}$, we see that, when $(b,\langle\sigma\rangle)$ runs through $\mathcal{T}$, the morphisms $\sigma$, as in (57), globalize to a morphism $\boldsymbol{\sigma}_{\mathcal{T}}: \mathcal{O}_{\mathbb{P}^{3}}(-a) \otimes L_{\mathcal{T}}^{\vee} \rightarrow \mathcal{E}_{\mathcal{T}}$ on $\mathcal{T}$, where $L_{\mathcal{T}}$ is the Grothendieck sheaf $\mathcal{O}_{\mathcal{T} / B}(1)$. Next, similar to (59), we define an open subset $\mathcal{S}$ of $\mathcal{T}$ as

$$
\mathcal{S}:=\left\{\left(b,\left[\sigma_{1}: \sigma_{2}\right]\right) \in \mathcal{T} \mid(i)\left(\sigma_{1}, \sigma_{2}\right): \mathcal{O}_{\mathbb{P}^{3}}(-a) \rightarrow E_{m} \text { is a subbundle morphism and }(i i) \sigma_{1}, \sigma_{2} \neq 0\right\} .
$$

Note that $\mathcal{S}$ is a nonempty set. (The proof mimics that of nonemptiness of the subset $M$ of $T$ given in paragraph after (59).) By the Base Change, the sheaf $F_{\widetilde{B}}=p_{\widetilde{B} *}\left(\mathcal{E}_{\widetilde{\mathbf{B}}}(a, 0)\right)$ is isomorphic to the sheaf $\left(F_{B}\right)_{\widetilde{B}}$. Therefore, from the definition of $\mathcal{T}$ it follows that the variety $\widetilde{Y}:=\mathbf{P}\left(F_{\widetilde{B}}^{\vee}\right)$ is isomorphic to $\widetilde{B} \times{ }_{B} \mathcal{T}$ :

$$
\widetilde{Y} \simeq \widetilde{B} \times_{B} \mathcal{T} \text {. }
$$

Thus by (69) and (70) we have $\widetilde{Y}=\left\{\left(b, \varphi_{1}, \varphi_{2},\left[\sigma_{1}: \sigma_{2}\right]\right) \mid\left(b, \varphi_{1}, \varphi_{2}\right) \in \widetilde{B},\left[\sigma_{1}: \sigma_{2}\right] \in P\left(H^{0}\left(E_{b}(a)\right)\right)\right\}$, and the natural projection $\widetilde{Y} \rightarrow \widetilde{B},(\beta,\langle\sigma\rangle) \mapsto \beta$ is a locally trivial $\mathbb{P}^{r-1}$-bundle. We now use (72) and the open subset $\mathcal{S}$ of $\mathcal{T}$ to define an open subset $Y$ of $\widetilde{Y}$ as

$$
Y:=\widetilde{B} \times_{B} \mathcal{S} .
$$

Here, $Y$ is a nonempty open in $\widetilde{Y}$ since $\mathcal{S}$ is nonempty. It follows that $Y$ is irreducible and dense in $\tilde{Y}$ since $\tilde{Y}$ is irreducible. In addition, using (71) and the above description of $\widetilde{Y}$ we obtain:

(74) $Y=\left\{\left(b, \varphi_{1}, \varphi_{2},\left[\sigma_{1}: \sigma_{2}\right]\right) \in \widetilde{Y} \mid(i)\left(\sigma_{1}, \sigma_{2}\right): \mathcal{O}_{\mathbb{P}^{3}}(-a) \rightarrow E_{m}\right.$ is a subbundle morphism and $\left.(i i) \sigma_{1}, \sigma_{2} \neq 0\right\}$.

The morphism $\boldsymbol{\sigma}_{\mathbf{Y}}:=\left(\boldsymbol{\sigma}_{\mathcal{T}}\right)_{\mathbf{Y}}$ is included in the universal monad on $\mathbf{Y}$ :

$$
\mathbf{A}_{\mathbf{Y}}: 0 \rightarrow \mathcal{O}_{\mathbb{P}^{3}}(-a) \otimes L_{Y}^{\vee} \stackrel{\sigma_{\mathbf{Y}}}{\longrightarrow} \mathcal{E}_{\mathbf{Y}} \stackrel{\boldsymbol{\sigma}_{\mathbf{Y}}^{t}}{\longrightarrow} \mathcal{O}_{\mathbb{P}^{3}}(a) \otimes L_{Y} \rightarrow 0,
$$

where $L_{Y}=\left(L_{\mathcal{T}}\right)_{Y}$ and $\boldsymbol{\sigma}_{Y}^{t}$ is the composition $\mathcal{E}_{\mathbf{Y}} \stackrel{\varphi_{\mathbf{Y}}}{\longrightarrow} \mathcal{E}_{\mathbf{Y}}^{\vee} \stackrel{\sigma_{\mathbf{Y}}^{\vee}}{\longrightarrow} \mathcal{O}_{\mathbb{P}^{3}}(a) \otimes L_{Y}$. By construction, for any point $(\beta,\langle\sigma\rangle) \in Y, \beta=\left(b, \varphi_{1}, \varphi_{2}\right)$, the restriction of the monad $\mathbf{A}_{\mathbf{Y}}$ onto $\mathbb{P}^{3} \times\{(\beta,\langle\sigma\rangle)\}$ is isomorphic to the monad $A_{E_{b}, \varphi_{1} \oplus \varphi_{2}, \sigma}$ in (42). Hence,

$$
\left.\mathcal{H}^{0}\left(\mathbf{A}_{\mathbf{Y}}\right)\right|_{\mathbb{P}^{3} \times\{(\beta,\langle\sigma\rangle)\}}=\mathcal{H}^{0}\left(A_{E_{b}, \varphi_{1} \oplus \varphi_{2}, \sigma}\right), \quad(\beta,\langle\sigma\rangle) \in Y, \quad \beta=\left(b, \varphi_{1}, \varphi_{2}\right) .
$$

Now consider the rank 2 the vector bundle $\mathbf{U}$ on $M$ defined in (50) and its associated principal frame bundle

$$
I:=\operatorname{Isom}\left(V_{2} \otimes \mathcal{O}_{M}, \mathbf{U}\right) \stackrel{\xi}{\rightarrow} M
$$


together with the tautological isomorphism $V_{2} \otimes \mathcal{O}_{I} \stackrel{\sim}{\rightarrow} \mathbf{U}_{I}$. Using this isomorphism and applying to (52) the functor $\boldsymbol{\xi}^{*}$ we obtain an isomorphism

$$
\mathbf{E}_{\mathbf{I}} \cong V_{2} \otimes \mathcal{O}_{\mathbf{I}} \oplus \mathbf{N}_{\mathbf{I}}
$$

Besides, by (50), we have a symplectic structure $\varphi_{\mathbf{I}}:=\left(\varphi_{\mathbf{M}}\right)_{\mathbf{I}}: \mathbf{E}_{\mathbf{I}} \stackrel{\simeq}{\rightarrow} \mathbf{E}_{\mathbf{I}}^{\vee}$ on $\mathbf{E}_{\mathbf{I}}$. This symplectic structure in view of (77) splits into a direct sum of two symplectic structures

$$
\varphi_{\mathbf{I}}=\varphi_{\mathbf{I}, 1} \oplus \varphi_{\mathbf{I}, 2}, \quad \varphi_{\mathbf{I}, 1}: V_{2} \otimes \mathcal{O}_{\mathbf{I}} \stackrel{\simeq}{\rightarrow} V_{2}^{\vee} \otimes \mathcal{O}_{\mathbf{I}}, \quad \varphi_{\mathbf{I}, 2}: \mathbf{N}_{\mathbf{I}} \stackrel{\simeq}{\rightarrow} \mathbf{N}_{\mathbf{I}}^{\vee}
$$

Remark that, by the defscription of the morphism $\Psi$ given in (49), we have $\Psi(M)=B$. Now, comparing (68)-(69) with (77)-(78), we obtain a morphism

$$
\Gamma: I \rightarrow \widetilde{B}, \quad x \mapsto\left(b, \varphi_{1}, \varphi_{2}\right), \quad b=\Psi(\xi(x)), \quad \varphi_{i}=\left.\varphi_{\mathbf{I}, i}\right|_{\mathbb{P}^{3} \times\{x\}}, \quad i=1,2,
$$

such that

$$
\mathbf{E}_{\mathbf{I}} \cong\left(\mathcal{E}_{\widetilde{\mathrm{B}}}\right)_{\mathbf{I}}, \quad \varphi_{\mathbf{I}} \cong\left(\varphi_{\widetilde{\mathrm{B}}}\right)_{\mathbf{I}},
$$

and these isomorphisms are compatible with the direct sum decompositions (77), (78) and (68). From (79) and the surjectivity of $\Psi$ it follows that $\Gamma$ is also surjective. Set

$$
X:=I \times_{M} S, \quad Y \stackrel{\Gamma_{Y}}{\longleftarrow} X \stackrel{\xi_{S}}{\longrightarrow} S, \quad F_{I}:=p_{I *}\left(\mathbf{E}_{\mathbf{I}}(a, 0)\right) .
$$

From (54), (80), the isomorphism $F_{\widetilde{B}} \simeq\left(F_{B}\right)_{\widetilde{B}}$ and the Base Change we obtain $F_{I} \simeq\left(F_{\widetilde{B}}\right)_{I}$, so that, in view of (72) and the equality $T=\mathbf{P}\left(F^{\vee}\right)$, the variety $\widetilde{X}:=\mathbf{P}\left(F_{X}^{\vee}\right)$ satisfies the isomorphisms

$$
I \times_{M} T \simeq \widetilde{X} \simeq I \times_{\widetilde{B}} \widetilde{Y} .
$$

The definition of $X$ (see (81)) and the left isomorphism (82) imply that there exists an open embedding $X \hookrightarrow \widetilde{X}$ such that $X=\widetilde{X} \times_{T} S$. Therefore, comparing the descriptions (74) and (59) of $Y$ and $S$ and using the right isomorphism (82), we obtain:

$$
X \simeq I \times_{\widetilde{B}} Y .
$$

This together with (80) implies that $\mathbf{E}_{\mathbf{X}} \cong\left(\mathcal{E}_{\mathbf{Y}}\right)_{\mathbf{X}}$. Moreover, since $X=I \times_{M} S$, we have

$$
\mathcal{A}_{\mathbf{X}} \cong\left(\mathbf{A}_{\mathbf{Y}}\right)_{\mathbf{X}}
$$

where the monads $\mathcal{A}$ and $\mathbf{A}_{\mathbf{Y}}$ were defined in (60) and (75), respectively. Consider the modular morphisms

$$
\Phi_{X}: X \rightarrow \mathcal{B}\left(a^{2}+1\right), \quad \Phi_{Y}: Y \rightarrow \mathcal{B}\left(a^{2}+1\right),
$$

defined by the (families of) sheaves $\mathcal{H}^{0}\left(\mathcal{A}_{\mathbf{X}}\right), \mathcal{H}^{0}\left(\mathbf{A}_{\mathbf{Y}}\right)$, respectively. From (84), (83) and (81) it follows that $\Phi_{X}$ factors through $\Gamma_{Y}$ and through $\xi_{S}$ as: $\Phi_{X}=\Phi_{Y} \circ \Gamma_{Y}=\Phi_{S} \circ \xi_{S}$. Here, $\Phi_{S}: S \rightarrow \mathcal{B}\left(a^{2}+1\right)$ is the modular morphism (66), $\xi_{S}$ in (81) is surjective by the surjectivity of $\xi$, and $\Gamma_{Y}$ is surjective as $\Gamma$ is surjective. Hence,

$$
\mathcal{G}(a, 1)_{0}=\Phi_{S}(S)=\Phi_{Y}(Y) .
$$

On the other hand, by Proposition 14, $\mathcal{G}(a, 1)_{0}$ is dense in $\overline{\mathcal{G}(a, 1)}$. We thus obtain

Proposition 15. Let $\Phi_{Y}: Y \rightarrow \mathcal{B}\left(a^{2}+1\right)$ be the modular morphism defined by the family of sheaves $\mathcal{H}^{0}\left(\mathbf{A}_{\mathbf{Y}}\right)$, where $\mathbf{A}_{\mathbf{Y}}$ is the monad (75). Then $\Phi_{Y}(Y)$ is dense in $\overline{\mathcal{G}(a, 1)}$.

\section{Series of Rational irReducible components of the moduli spaces $\mathcal{B}\left(a^{2}+1\right)$}

Consider the variety $Y$ defined in (73). We first will relate to $Y$ a new variety $\mathcal{P}_{a}$, together with a natural projection $\pi: Y \rightarrow \mathcal{P}_{a}$. In this section we will relate the morphism $\pi$ to the modular morphism $\Phi_{Y}: Y \rightarrow \mathcal{B}\left(a^{2}+1\right)$ (for the precise formulation see Theorem (18). For this, take any point $y \in Y$. By (74), $y$ is a collection of data

$$
y=\left(b, \varphi_{1}, \varphi_{2},\left[\sigma_{1}: \sigma_{2}\right]\right),
$$

where (i) $b \in B$, (ii) $\varphi_{1}: V_{2} \otimes \mathcal{O}_{\mathbb{P}^{3}} \stackrel{\simeq}{\rightarrow} V_{2}^{\vee} \otimes \mathcal{O}_{\mathbb{P}^{3}}$ and $\varphi_{2}: N_{b} \stackrel{\simeq}{\rightarrow} N_{b}^{\vee}$ are symplectic isomorphisms:

(87) $\varphi_{1} \in H^{0}\left(\wedge^{2}\left(V_{2} \otimes \mathcal{O}_{\mathbb{P}^{3}}\right)^{\vee}\right) \backslash\{0\}=\wedge^{2} V_{2}^{\vee} \backslash\{0\} \cong \mathbf{k}^{\times}, \quad \varphi_{2} \in H^{0}\left(\wedge^{2} N_{b}^{\vee}\right) \backslash\{0\}=H^{0}\left(\mathcal{O}_{\mathbb{P}^{3}}\right) \backslash\{0\} \cong \mathbf{k}^{\times}$,

(iii) $\sigma_{1}$ and $\sigma_{2}$ are:

$$
0 \neq \sigma_{1} \in H^{0}\left(V_{2} \otimes \mathcal{O}_{\mathbb{P}^{3}}(a)\right)=\operatorname{Hom}\left(V_{2}^{\vee}, W_{a}\right), \quad W_{a}:=H^{0}\left(\mathcal{O}_{\mathbb{P}^{3}}(a)\right), \quad 0 \neq \sigma_{2} \in H^{0}\left(N_{b}(a)\right),
$$


(iv) $\sigma=\left(\sigma_{1}, \sigma_{2}\right): \mathcal{O}_{\mathbb{P}^{3}}(-a) \rightarrow V_{2} \otimes \mathcal{O}_{\mathbb{P}^{3}} \oplus N_{b}$ is a subbundle morphism. In $\operatorname{Hom}\left(V_{2}^{\vee}, W_{a}\right)$ consider an open subset $\operatorname{Hom}^{\text {in }}\left(V_{2}^{\vee}, W_{a}\right):=\left\{\sigma_{1} \in \operatorname{Hom}\left(V_{2}^{\vee}, W_{a}\right) \mid \sigma_{1}: V_{2}^{\vee} \rightarrow W_{a}\right.$ is a monomorphism $\}$. One can easily see (use the argument in paragraph after (59)) that

$$
\operatorname{Hom}^{\text {in }}\left(V_{2}^{\vee}, W_{a}\right)=\left\{\sigma_{1} \in \operatorname{Hom}\left(V_{2}^{\vee}, W_{a}\right) \mid \operatorname{dim}\left(\sigma_{1}\right)_{0}=1\right\} .
$$

Besides, note that the group $G L\left(V_{2}\right)$ naturally acts on $\operatorname{Hom}^{\mathrm{in}}\left(V_{2}^{\vee}, W_{a}\right)$ via its action on $V_{2}^{\vee}$, and we have an isomorphism

$$
\operatorname{Hom}^{\text {in }}\left(V_{2}^{\vee}, W_{a}\right) / G L\left(V_{2}\right) \stackrel{\simeq}{\rightarrow} G r\left(2, W_{a}\right)
$$

and the factorization morphism

$$
\tau_{1}: \operatorname{Hom}^{\mathrm{in}}\left(V_{2}^{\vee}, W_{a}\right) \rightarrow \operatorname{Gr}\left(2, W_{a}\right), \quad \sigma_{1} \mapsto \operatorname{im}\left(\sigma_{1}: V_{2}^{\vee} \hookrightarrow W_{a}\right) .
$$

Next, as it was mentioned in Section廿4(see paragraph after (59)), the set $H^{0}\left(N_{b}(a)\right)^{*}:=\left\{\sigma_{2} \in H^{0}\left(N_{b}(a)\right) \mid \operatorname{dim}\left(\sigma_{2}\right)_{0}\right.$ $=1\}$ is open dense in $H^{0}\left(N_{b}(a)\right)$. Besides, it is clearly invariant under the action of the group $\operatorname{Aut}\left(N_{b}(a)\right)=\mathbf{k}^{\times}$. (Recall that the null correlation bundle $N_{b}$ is stable and therefore simple, i. e., $\operatorname{End}\left(N_{b}(a)\right)=\mathbf{k} \cdot$ id.) Hence,

$$
P\left(H^{0}\left(N_{b}(a)\right)\right)^{*}=H^{0}\left(N_{b}(a)\right)^{*} / \operatorname{Aut}\left(N_{b}(a)\right) \stackrel{\text { open }}{\hookrightarrow} P\left(H^{0}\left(N_{b}(a)\right)\right) \simeq \mathbb{P}^{r},
$$

where $r=2\left(\begin{array}{c}a+3 \\ 3\end{array}\right)-a-3$, and we have the factorization morphism

$$
\tau_{2}: H^{0}\left(N_{b}(a)\right)^{*} \rightarrow P\left(H^{0}\left(N_{b}(a)\right)\right)^{*}, \quad \sigma_{2} \mapsto\left\langle\sigma_{2}\right\rangle .
$$

Now the above condition (iv) imposed on $\left(\sigma_{1}, \sigma_{2}\right)$ can be rewritten in the form:

$$
\left(\sigma_{1}, \sigma_{2}\right) \in H_{b, a}:=\left\{\left(\sigma_{1}, \sigma_{2}\right) \in \operatorname{Hom}^{\mathrm{in}}\left(V_{2}^{\vee}, W_{a}\right) \times H^{0}\left(N_{b}(a)\right)^{*} \mid\left(\sigma_{1}\right)_{0} \cap\left(\sigma_{2}\right)_{0}=\emptyset\right\} .
$$

Clearly, $H_{b, a}$ is a dense open subset of $\operatorname{Hom}^{\text {in }}\left(V_{2}^{\vee}, W_{a}\right) \times H^{0}\left(N_{b}(a)\right)^{*}$. This subset is invariant under the action of the group $\mathbf{k}^{\times}$by homotheties. Therefore, denoting $P\left(H_{b, a}\right):=H_{b, a} / \mathbf{k}^{\times}$and using (90) and (92), we obtain the factorization morphism

$$
\tau: P\left(H_{b, a}\right) \rightarrow G r\left(2, W_{a}\right) \times \mathbb{P}\left(H^{0}\left(N_{b}(a)\right)\right)^{*}, \quad\left[\sigma_{1}: \sigma_{2}\right] \mapsto\left(\tau_{1}\left(\sigma_{1}\right), \tau_{2}\left(\sigma_{2}\right)\right) .
$$

To globalize the above pointwise (w.r.t. $b \in B)$ constructions over $B$, set $\mathcal{K}:=p_{B *}(\mathcal{N}(a, 0))$. The variety $\mathbf{P}\left(\mathcal{K}^{\vee}\right)$ has the description $\mathbf{P}\left(\mathcal{K}^{\vee}\right)=\left\{\left(b,\left\langle\sigma_{2}\right\rangle\right) \mid b \in B,\left\langle\sigma_{2}\right\rangle \in P\left(H^{0}\left(N_{b}(a)\right)\right)\right\}$. Consider its dense open subset

$$
\Pi_{a}:=\left\{\left(b,\left\langle\sigma_{2}\right\rangle\right) \in \mathbf{P}\left(\mathcal{K}^{\vee}\right) \mid\left\langle\sigma_{2}\right\rangle \in \mathbb{P}\left(H^{0}\left(N_{b}(a)\right)\right)^{*}\right\}
$$

and set

$$
\mathcal{G}_{a}:=\operatorname{Gr}\left(2, W_{a}\right) \times \Pi_{a}, \quad \mathcal{G}_{a}=\left\{\left(b, V,\left\langle\sigma_{2}\right\rangle\right) \mid V \in G r\left(2, W_{a}\right),\left(b,\left\langle\sigma_{2}\right\rangle\right) \in \Pi_{a}\right\} .
$$

By construction, $\mathcal{G}_{a}$ is a rational variety. Next, remark that, comparing the definitions (71) and (93) of $\mathcal{S}$ and $H_{b, a}$, we obtain

$$
\mathcal{S}=\left\{\left(b,\left[\sigma_{1}: \sigma_{2}\right]\right) \mid b \in B,\left[\sigma_{1}: \sigma_{2}\right] \in \mathbb{P}\left(H_{b, a}\right)\right\} .
$$

Thus, by (94), we have a well-defined morphism

$$
\tau: \mathcal{S} \rightarrow \mathcal{G}_{a},\left(b,\left[\sigma_{1}: \sigma_{2}\right]\right) \mapsto\left(b, \tau_{1}\left(\sigma_{1}\right), \tau_{2}\left(\sigma_{2}\right)\right) .
$$

Consider the group $\tilde{G}=G L\left(V_{2}\right) \times \mathbf{k}^{\times}$, its normal subgroup $G^{\prime}=\left\{\left(\rho \cdot \operatorname{id}_{V_{2}}, \rho\right) \mid \rho \in \mathbf{k}^{\times}\right\}$, and let

$$
G=\tilde{G} / G^{\prime}
$$

be the factor group. We will use the following notation for elements of $G:\left[g_{1}: \lambda\right]:=\left(g_{1}, \lambda\right) G^{\prime}=\left\{\left(\rho g_{1}, \rho \lambda\right) \mid \rho \in\right.$ $\left.\mathbf{k}^{\times}\right\}, \quad\left(g_{1}, \lambda\right) \in \tilde{G}$. The group $G$ naturally acts on $\mathcal{S}$ as:

$$
a_{\mathcal{S}}: \mathcal{S} \times G \rightarrow \mathcal{S}, \quad\left(\left(b,\left[\sigma_{1}: \sigma_{2}\right]\right),\left[g_{1}: \lambda\right]\right) \mapsto\left(b,\left[g_{1} \circ \sigma_{1}: \lambda \sigma_{2}\right]\right),
$$

and formulas (89)-(96) show that $\mathcal{G}_{a}=\mathcal{S} / G$ and the morphism $\tau: \mathcal{S} \rightarrow \mathcal{G}_{a}$ in (96) is the quotient morphism for this action and it is a principal $G$-bundle. Therefore in view of (53) we have:

$$
\operatorname{dim} \mathcal{G}_{a}=\operatorname{dim} P\left(H_{b, a}\right)+\operatorname{dim} B-\operatorname{dim} G=4\left(\begin{array}{c}
a+3 \\
3
\end{array}\right)-a-2 .
$$


The principal $G$-bundle $\mathcal{S} \stackrel{\tau}{\rightarrow} \mathcal{G}_{a}$ by construction is locally trivial, hence there exists an open dense subset $U$ of $\mathcal{G}_{a}$ and a section $U \stackrel{s}{\hookrightarrow} \mathcal{S}$ of the projection $\left.\tau\right|_{\tau^{-1}(U)}: \tau^{-1}(U) \rightarrow U:$

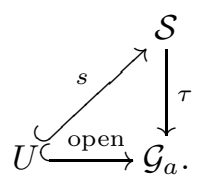

Here $U$ is rational since $\mathcal{G}_{a}$ is rational as it was mentioned above.

Now consider the variety $\mathbf{P}\left(\wedge^{2}\left(V_{2} \otimes \mathcal{O}_{\mathbf{B}}\right) \oplus \wedge^{2} \mathcal{N}\right)$ together with the embeddings

$$
\mathbf{P}\left(\wedge^{2}\left(V_{2} \otimes \mathcal{O}_{\mathbf{B}}\right)\right) \hookrightarrow \mathbf{P}\left(\wedge^{2}\left(V_{2} \otimes \mathcal{O}_{\mathbf{B}}\right) \oplus \wedge^{2} \mathcal{N}\right) \hookleftarrow \mathbf{P}\left(\wedge^{2} \mathcal{N}\right)
$$

and denote $P \widetilde{B}:=\mathbf{P}\left(\wedge^{2}\left(V_{2} \otimes \mathcal{O}_{\mathbf{B}}\right) \oplus \wedge^{2} \mathcal{N}\right) \backslash\left\{\mathbf{P}\left(\wedge^{2}\left(V_{2} \otimes \mathcal{O}_{\mathbf{B}}\right)\right) \sqcup \mathbf{P}\left(\wedge^{2} \mathcal{N}\right)\right\}$. By construction, the natural projection $P \widetilde{B} \rightarrow B$ is a locally trivial fibration with fiber

$$
\mathbf{F} \simeq \mathbb{P}^{1} \backslash\{2 \text { points }\} .
$$

Using the description (69) of the varieties $B_{1}, B_{2}$ and the notation (58) in which we put $\varphi_{1}, \varphi_{2}$ in place of $\sigma_{1}, \sigma_{2}$, we obtain $\mathbb{P} \widetilde{B}=\left\{\left(b,\left[\varphi_{1}: \varphi_{2}\right]\right) \mid\left(b, \varphi_{i}\right) \in B_{i}, i=1,2\right\}$. Remark that the group $\mathbf{k}^{\times}$naturally acts on $\widetilde{B}$ as

$$
\widetilde{B} \times \mathbf{k}^{\times} \rightarrow \widetilde{B}, \quad\left(\left(b, \varphi_{1}, \varphi_{2}\right), \lambda\right) \mapsto\left(b, \lambda \varphi_{1}, \lambda \varphi_{2}\right),
$$

(here we use the description (69) of $\widetilde{B}$ ), so that

$$
P \widetilde{B}=\widetilde{B} / \mathbf{k}^{\times}
$$

and we have the factorization morphism

$$
\pi_{\widetilde{B}}: \widetilde{B} \rightarrow P \widetilde{B}, \quad\left(b, \varphi_{1}, \varphi_{2}\right) \mapsto\left(b,\left[\varphi_{1}: \varphi_{2}\right]\right) .
$$

Consider the varieties $P Y:=P \widetilde{B} \times_{B} \mathcal{S}=\left\{\left(b,\left[\varphi_{1}: \varphi_{2}\right],\left[\sigma_{1}: \sigma_{2}\right]\right) \mid\left(b,\left[\varphi_{1}: \varphi_{2}\right]\right) \in P \widetilde{B},\left(b,\left[\sigma_{1}: \sigma_{2}\right]\right) \in \mathcal{S}\right\}$ and $\mathcal{P}_{a}:=P \widetilde{B} \times_{B} \mathcal{G}_{a}=\left\{\left(b,\left[\varphi_{1}: \varphi_{2}\right], V,\left\langle\sigma_{2}\right\rangle\right) \mid\left(b,\left[\varphi_{1}: \varphi_{2}\right]\right) \in P \widetilde{B},\left(b, V,\left\langle\sigma_{2}\right\rangle\right) \in \mathcal{G}_{a}\right\}$, where $\mathcal{G}_{a}$ was defined in (95). From (99) and (101) we have

$$
\operatorname{dim} \mathcal{P}_{a}=\operatorname{dim} \mathcal{G}_{a}+\operatorname{dim} \mathbf{F}=4\left(\begin{array}{c}
a+3 \\
3
\end{array}\right)-a-1
$$

Note that the local triviality of the fibration $P \mathcal{B} \rightarrow B$ implies that the natural projection

$$
p r_{Y}: P Y \rightarrow \mathcal{S}
$$

is a locally trivial fibration with fiber $\mathbf{F}$ given in (101).

The morphism $\pi_{\widetilde{B}}$ in (104) induces the morphism

$$
\pi_{Y}: Y \rightarrow P Y,\left(b, \varphi_{1}, \varphi_{2},\left[\sigma_{1}: \sigma_{2}\right]\right) \mapsto\left(b,\left[\varphi_{1}: \varphi_{2}\right],\left[\sigma_{1}: \sigma_{2}\right]\right),
$$

and from (102)-(104) it follows that $\pi_{Y}$ is a factorization morphism of the following $\mathbf{k}^{\times}$-action on $Y$ :

$$
a_{Y}: Y \times \mathbf{k}^{\times} \rightarrow Y, \quad\left(\left(b, \varphi_{1}, \varphi_{2},\left[\sigma_{1}: \sigma_{2}\right]\right), \lambda\right) \mapsto\left(b, \lambda \varphi_{1}, \lambda \varphi_{2},\left[\sigma_{1}: \sigma_{2}\right]\right) .
$$

Respectively, the morphism $\tau: Y_{a} \rightarrow \mathcal{G}_{a}$ defined in (96) induces a morphism

$$
\tau_{Y}: P Y \rightarrow \mathcal{P}_{a},\left(b,\left[\varphi_{1}: \varphi_{2}\right],\left[\sigma_{1}: \sigma_{2}\right]\right) \mapsto\left(b,\left[\varphi_{1}: \varphi_{2}\right], \tau_{1}\left(\sigma_{1}\right), \tau_{2}\left(\sigma_{2}\right)\right) .
$$

Define the morphism $\pi: Y \rightarrow \mathcal{P}_{a}$ as the composition

$$
\pi=\tau_{Y} \circ \pi_{Y}: Y \rightarrow \mathcal{P}_{a}, \quad\left(b, \varphi_{1}, \varphi_{2},\left[\sigma_{1}: \sigma_{2}\right]\right) \mapsto\left(b,\left[\varphi_{1}: \varphi_{2}\right], \tau_{1}\left(\sigma_{1}\right), \tau_{2}\left(\sigma_{2}\right)\right) .
$$

We will now proceed to the study of the fibers of the morphism $\pi$.

Definition 16. Introduce on $Y$ the following equivalence relation:

$$
y=\left(b, \varphi_{1}, \varphi_{2},\left[\sigma_{1}: \sigma_{2}\right]\right) \sim\left(\tilde{b}, \tilde{\varphi}_{1}, \tilde{\varphi}_{2},\left[\tilde{\sigma}_{1}: \tilde{\sigma}_{2}\right]\right)=\tilde{y}
$$

if there exists an isomorphism of symplectic monads $A_{y}$ and $A_{\tilde{y}}$, $i$. e., a commutative diagram with rows $A_{y}$ and $A_{\tilde{y}}$ :

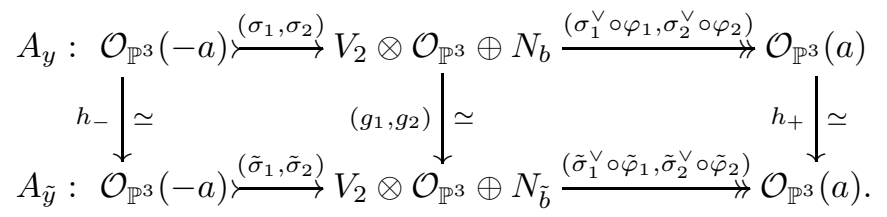


We denote by $[y]=\left[b, \varphi_{1}, \varphi_{2},\left[\sigma_{1}: \sigma_{2}\right]\right]$ the equivalence class of a point $y=\left(b, \varphi_{1}, \varphi_{2},\left[\sigma_{1}: \sigma_{2}\right]\right) \in Y$ under this equivalence relation.

Note that, in diagram (112), one has

$$
g_{1} \in \operatorname{Isom}\left(V_{2} \otimes \mathcal{O}_{\mathbb{P}^{3}}, V_{2} \otimes \mathcal{O}_{\mathbb{P}^{3}}\right) \cong G L\left(V_{2}\right) ;
$$

and $g_{2} \in \operatorname{Isom}\left(N_{b}, N_{\tilde{b}}\right)$ which in view of the stability of $N_{b}$ implies that

$$
b=\tilde{b}, \quad g_{2}=\lambda \cdot \operatorname{id}_{N_{b}}, \quad \lambda \in \mathbf{k}^{\times} ;
$$

besides, the isomorphisms $h_{-}, h_{+}$are multiplications by some constants $\mu, \nu \in \mathbf{k}^{\times}$, respectively:

$$
h_{-}=\mu \cdot \operatorname{id}_{\mathcal{O}_{\mathbb{P} 3}(-a)}, \quad h_{+}=\nu \cdot \operatorname{id}_{\mathcal{O}_{\mathbb{P} 3}(a)} .
$$

Furthermore, in view of (87), (113), (114) and the symplecticity of $\varphi_{1}, \varphi_{2}$, we have in (112)

$$
\tilde{\varphi}_{1}=\lambda_{1} \varphi_{1}, \quad \tilde{\varphi}_{2}=\lambda_{2} \varphi_{2}, \quad \lambda_{1}, \lambda_{2} \in \mathbf{k}^{\times}, \quad g_{1}^{\vee} \circ \varphi_{1} \circ g_{1}=\operatorname{det}\left(g_{1}\right) \varphi_{1}, \quad g_{2}^{\vee} \circ \varphi_{2} \circ g_{2}=\lambda^{2} \varphi_{2} .
$$

The leftmost square of diagram (112) together with (115) impliies:

$$
\tilde{\sigma}_{1}=\frac{1}{\mu} g_{1} \circ \sigma_{1}, \quad \tilde{\sigma}_{2}=\frac{\lambda}{\mu} \sigma_{2} .
$$

Respectively, the rightmost square of diagram (112) yields $\nu \sigma_{1}^{\vee} \circ \varphi_{1}=\tilde{\sigma}_{1}^{\vee} \circ \tilde{\varphi}_{1} \circ g_{1}, \quad \nu \sigma_{2}^{\vee} \circ \varphi_{2}=\lambda \tilde{\sigma}_{2}^{\vee} \circ \tilde{\varphi}_{2}$. Substituting (115)-(117) into the last equalities we obtain the relations $\nu=\frac{\lambda_{1} \operatorname{det}\left(g_{1}\right)}{\mu}$ and $\nu=\frac{\lambda_{2} \lambda^{2}}{\mu}$. Whence $\lambda_{1} \operatorname{det}\left(g_{1}\right)=\lambda_{2} \lambda^{2}$. This relation shows that the $G$-action (98) on $\mathcal{S}$ lifts to the following $G$-action on $P Y$ :

$$
a_{P Y}: P Y \times G \rightarrow P Y, \quad\left(\left(b,\left[\varphi_{1}: \varphi_{2}\right],\left[\sigma_{1}: \sigma_{2}\right]\right),\left[g_{1}: \lambda\right]\right) \mapsto\left(b,\left[\frac{\varphi_{1}}{\operatorname{det}\left(g_{1}\right)}: \frac{\varphi_{2}}{\lambda^{2}}\right],\left[g_{1} \circ \sigma_{1}: \lambda \sigma_{2}\right]\right) .
$$

Thus, $\mathcal{P}_{a}=P Y / G$ and the morphism

$$
\tau_{Y}: P Y \rightarrow \mathcal{P}_{a}
$$

in (109) is the quotient morphism for this action and it is a locally trivial principal $G$-bundle. We therefore have a commutative diagram

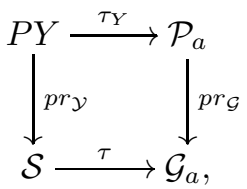

where $p r_{\mathcal{G}}$ is a natural projection. Since by (106) the morphism $p r_{\mathcal{Y}}: P Y \rightarrow \mathcal{S}$ is a locally trivial fibration with fibre $\mathbf{F}$, the open subset $U$ of $\mathcal{G}_{a}$ and the section $U \stackrel{s}{\hookrightarrow} \mathcal{S}$ in the diagram (100), after possible shrinking $U$, can be lifted to an open section $\mathbf{F} \times U \stackrel{\tilde{s}}{\leftrightarrow} P Y$ of the projection $\tau_{Y}: P Y \rightarrow \mathcal{P}_{a}$ :

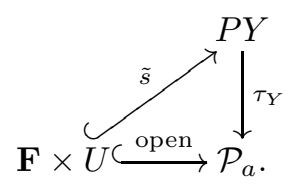

Since $\mathbf{F}$ is rational by (101) and $U$ is rational, it follows that

$$
\mathcal{P}_{a} \text { is rational. }
$$

Next, from (107)-(108), (118) and (119) it follows that the morphism $\pi: Y \rightarrow \mathcal{P}_{a}$ in (110) is the quotient morphism of the following action of the group $\bar{G}:=G \times \mathbf{k}^{\times}$on $Y$, where $G=\widetilde{G} / G^{\prime}$ was defined in (97):

(121) $a_{Y}: Y \times \bar{G} \rightarrow Y, \quad\left(\left(b, \varphi_{1}, \varphi_{2},\left[\sigma_{1}: \sigma_{2}\right]\right),\left(\left[g_{1}: \lambda\right], \mu\right)\right) \mapsto\left(b, \frac{\mu \varphi_{1}}{\operatorname{det}\left(g_{1}\right)}, \frac{\mu \varphi_{2}}{\lambda^{2}},\left[g_{1} \circ \sigma_{1}: \lambda \sigma_{2}\right]\right), \quad \bar{G}=G \times \mathbf{k}^{\times}$.

Moreover,

$$
\pi: Y \rightarrow \mathcal{P}_{a}=Y / \bar{G} \text { is a principal } \bar{G} \text {-bundle, }
$$

and computations (113)-(118) show that the equivalence class $[y]$ of any point $y \in Y$ is the $\bar{G}$-orbit of $y$ :

$$
[y]=a_{Y}(\{y\} \times \bar{G})=\pi^{-1}(\pi(y)), \quad y \in Y .
$$

In other words, $\mathcal{P}_{a}$ is the set of equivalence classes of points of $Y$ :

$$
\mathcal{P}_{a}=\{[y] \mid y \in Y\} .
$$


Remark that, by Corollary 2 , the equality $[y]=[\tilde{y}]$, i. e. the isomorphism of symplectic monads $A_{y}$ and $A_{\tilde{y}}$ in (112) is equivalent to the isomorphism of their cohomology rank 2 bundles as symplectic bundles $\left(\mathcal{H}^{0}\left(A_{y}\right), \psi_{y}\right)$ and $\left(\mathcal{H}^{0}\left(A_{\tilde{y}}\right), \psi_{\tilde{y}}\right)$, i. e., to the commutativity of the diagram

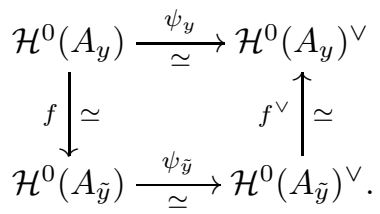

Here $\psi_{y}$, respectively, $\psi_{\tilde{y}}$, is a symplectic isomorphism induced by the symplectic isomorphism of the monad $A_{y}$ with its dual $A_{y}^{\vee}$, respectively, of $A_{\tilde{y}}$ with $A_{\tilde{y}}^{\vee}$. Thus, denoting by $\left[\mathcal{H}^{0}\left(A_{y}\right), \psi_{y}\right]$ the isomorphism class of the pair $\left(\mathcal{H}^{0}\left(A_{y}\right), \psi_{y}\right)$, we have:

$$
[y]=\left[\mathcal{H}^{0}\left(A_{y}\right), \psi_{y}\right]=\left[\mathcal{H}^{0}\left(A_{y}\right)\right] .
$$

This together with (122)-(124) shows that the modular morphism

$$
\Phi_{Y}: Y \rightarrow \mathcal{B}\left(a^{2}+1\right), \quad y \mapsto\left[\mathcal{H}^{0}\left(A_{y}\right)\right]
$$

factors through an injective map $\Theta: \mathcal{P}_{a} \rightarrow \mathcal{B}\left(a^{2}+1\right)$, i. e.

$$
\Phi_{Y}=\Theta \circ \pi \text {. }
$$

Since $Y$ is clearly smooth, the map $\Theta$ is actually a morphism. This outcomes from the following well known general result. (For the convenience of the reader we give its proof here.)

Lemma 17. Let $X, Y, Z$ be quasiprojective varieties with $Y$ smooth, and let $a: X \rightarrow Y$ and $b: X \rightarrow Z$ be morphisms such that $a$ is surjective and $b$ is constant on the fibers of $a$. Then there exists a morphism $f: Y \rightarrow Z$ such that $b=f \circ a$.

Proof. Consider the morphism $g: X \rightarrow Y \times Z, x \mapsto(a(x), b(x))$, and let $Y \stackrel{a^{\prime}}{\longleftarrow} Y \times Z \stackrel{b^{\prime}}{\rightarrow} Z$ be the projections onto factors so that $a=a^{\prime} \circ g$ and $b=b^{\prime} \circ g$. Since $b$ is constant on the fibers of $p$, it follows that $\tilde{a}:=\left.a^{\prime}\right|_{g(X)}: g(X) \rightarrow Y$ is a bijection. Therefore, as $Y$ is smooth, $\tilde{a}$ is an isomorphism (see, e.g., [S, Ch.2, Section 4.4, Thm. 2.16]). The desired morphism $f$ is now the composition $f=b^{\prime} \circ \tilde{a}^{-1}$.

Now Proposition 15 together with (105), (120), (122) and (127) yields

Theorem 18. There exists an injective morphism $\Theta: \mathcal{P}_{a} \hookrightarrow \mathcal{B}\left(a^{2}+1\right)$ such that the modular morphism $\Phi_{Y}$ : $Y \rightarrow \mathcal{B}\left(a^{2}+1\right)$ factorizes as

$$
\Phi_{Y}: Y \stackrel{\pi}{\rightarrow} \mathcal{P}_{a} \stackrel{\Theta}{\hookrightarrow} \mathcal{B}\left(a^{2}+1\right),
$$

where $\pi: Y \rightarrow \mathcal{P}_{a}$ is a principal $\bar{G}$-bundle with the group $\bar{G}$ defined in (121). The variety $\overline{\mathcal{G}(a, 1)}$ containing the rational variety $\mathcal{G}(a, 1)_{0}=\Theta\left(\mathcal{P}_{a}\right)$ as a dense subset is rational of dimension $4\left(\begin{array}{c}a+3 \\ 3\end{array}\right)-a-1$.

We next obtain the following important formula.

Lemma 19. For every $[\mathcal{E}] \in \mathcal{G}(a, 1)_{0}$ with $a \geq 2$, it holds

$$
h^{1}(\mathcal{E} n d(\mathcal{E}))=4 \cdot\left(\begin{array}{c}
a+3 \\
3
\end{array}\right)-a-1+\varepsilon(a),
$$

where $\varepsilon(a)=1$ when $a=3$, and $\varepsilon(a)=0$ when $a \neq 3$.

Proof. Since $\mathcal{E}$ is a self dual rank 2 bundle, we have $\mathcal{E} n d(\mathcal{E}) \simeq S^{2} \mathcal{E} \oplus \Lambda^{2} \mathcal{E}=S^{2} \mathcal{E} \oplus \mathcal{O}_{\mathbb{P}^{3}}$, thus $h^{1}(\mathcal{E} n d(\mathcal{E}))=h^{1}\left(S^{2} \mathcal{E}\right)$. We will compute the latter.

By the definition of $\mathcal{G}(a, 1)_{0}$ (see Proposition 14,(ii), (59) and (61)), $\mathcal{E}$ is the cohomology of a complex $M^{\bullet}$ with terms $M^{-1}=\mathcal{O}_{\mathbb{P}^{3}}(-a), M^{0}=E \simeq \mathcal{O}_{\mathbb{P}^{3}}^{\oplus 2} \oplus N, M^{1}=\mathcal{O}_{\mathbb{P}^{3}}(a)$. Proceed to the double complex $M^{\bullet} \otimes M^{\bullet}$ and to its total complex $T^{\bullet}$. The symmetric part of $T^{\bullet}$ is the $\operatorname{monad} 0 \rightarrow E(-a) \rightarrow S^{2} E \oplus \mathcal{O}_{\mathbb{P}^{3}} \rightarrow E(a) \rightarrow 0$, whose cohomology sheaf is isomorphic to $S^{2} \mathcal{E}$. Therefore this monad can be broken into two short exact sequences

$$
0 \rightarrow K \rightarrow S^{2} E \oplus \mathcal{O}_{\mathbb{P}^{3}} \rightarrow E(a) \rightarrow 0 \quad \text { and } \quad 0 \rightarrow E(-a) \rightarrow K \rightarrow S^{2} \mathcal{E} \rightarrow 0 .
$$

Since $h^{0}(E(-a))=h^{0}\left(S^{2} \mathcal{E}\right)=0$, it follows that $h^{0}(K)=0$; in addition, $h^{1}(E(a))=h^{2}\left(S^{2} E \oplus \mathcal{O}_{\mathbb{P}^{3}}\right)=0($ use Proposition 5) implies that $h^{2}(K)=0$. It then follows in view of the splitting $E \simeq \mathcal{O}_{\mathbb{P} 3}^{\oplus 2} \oplus N$ that

$$
h^{1}\left(S^{2} \mathcal{E}\right)=h^{1}(K)+h^{2}(E(-a))=h^{1}(K)+\varepsilon(a), \quad \varepsilon(a):=h^{2}(N(-a)),
$$


since $h^{1}(E(-a))=0$ for $a \geq 2$.

To complete our calculation, consider the exact sequence

$$
0 \rightarrow H^{0}\left(S^{2} E \oplus \mathcal{O}_{\mathbb{P}^{3}}\right) \rightarrow H^{0}(E(a)) \rightarrow H^{1}(K) \rightarrow H^{1}\left(S^{2} E \oplus \mathcal{O}_{\mathbb{P}^{3}}\right) \rightarrow 0 .
$$

Since $h^{0}\left(S^{2} E \oplus \mathcal{O}_{\mathbb{P}^{3}}\right)=4$ and $h^{1}\left(S^{2} E \oplus \mathcal{O}_{\mathbb{P}^{3}}\right)=5$ by Proposition 5 , we conclude that

$$
h^{1}(K)=h^{0}(E(a))+1=h^{0}(N(a))+V_{2} \otimes h^{0}\left(\mathcal{O}_{\mathbb{P}^{3}}(a)\right)+1,
$$

which, together with the equality in equation (129), yields the desired formula.

It is interesting to observe that the right hand side of the formula in Lemma 19yields the value of $h^{1}(\mathcal{E} n d(\mathcal{E}))$ expected by the deformation theory when $a=2$ and $a=3$, respectively 37 and 77; when $a \geq 4$, one can check that $4 \cdot\left(\begin{array}{c}a+3 \\ 3\end{array}\right)-a-1>8\left(a^{2}+1\right)-3$.

Noting that, in view of Theorem [18, the dimension of $\overline{\mathcal{G}(a, 1)}$ equals $h^{1}(\mathcal{E} n d(E))$ for $a=2$ and $a \geq 4$, as calculated in Lemma 19, and using Proposition 14, we have therefore completed the proof of the first main result of this paper.

Theorem 20. For $a=2$ and $a \geq 4$, the rank 2 bundles given as cohomology of monads of the form

$$
0 \rightarrow \mathcal{O}_{\mathbb{P}^{3}}(-a) \oplus \mathcal{O}_{\mathbb{P}^{3}}(-1) \rightarrow V_{6} \otimes \mathcal{O}_{\mathbb{P}^{3}} \rightarrow \mathcal{O}_{\mathbb{P}^{3}}(1) \oplus \mathcal{O}_{\mathbb{P}^{3}}(a) \rightarrow 0
$$

fill out a dense subset of a rational irreducible component of $\mathcal{B}\left(a^{2}+1\right)$ of dimension

$$
4 \cdot\left(\begin{array}{c}
a+3 \\
3
\end{array}\right)-a-1
$$

In particular, for the case $a=2$, we conclude that rank 2 bundles given as cohomology of monads of the form (5) yield a dense subset of an irreducible component of $\mathcal{B}(5)$ with expected dimension 37.

\section{Cohomology bundle $\mathcal{E}$ of the monad of type (6) and the Related Reflexive Sheaf $\mathcal{F}$}

Consider the set

$$
\mathcal{H}=\{[\mathcal{E}] \in \mathcal{B}(5) \mid \mathcal{E} \text { is cohomology of a monad of type ([6) }\} .
$$

It is known that $\mathcal{H} \neq \emptyset$ - see [21, Table $5.3, c_{2}=5$, Case (2).ii)]. Note that the set $\mathcal{H}$ is a constructible subset of $\mathcal{B}(5)$, as well as $\mathcal{G}(2,1)$ (see Remark after Proposition 7). The aim of this and the subsequent sections is to prove

Theorem 21. The set $\mathcal{H}$ satisfies the condition $\operatorname{dim}(\mathcal{H} \backslash(\mathcal{G}(2,1) \cap \mathcal{H})) \leq 36$. Its closure in $\mathcal{B}(5)$ does not constitute a component of $\mathcal{B}(5)$.

In this section we will relate the vector bundle $[\mathcal{E}] \in \mathcal{H} \backslash(\mathcal{G}(2,1) \cap \mathcal{H})$ to a rank 2 reflexive sheaf $\mathcal{F}$ with Chern classes $c_{1}(\mathcal{F})=0, c_{2}(\mathcal{F})=2$ and $c_{3}(\mathcal{F})=2 k, 0 \leq k \leq 6$, which appears as a middle cohomology of a left-exact complex $K^{\bullet}$ (see (154) $)$ induced by the monad of type (6) defining $\mathcal{E}$. This relation will be established in Proposition 25. We will then use it in Section 7 to prove Theorem 21,

Let $[\mathcal{E}] \in \mathcal{H} \backslash(\mathcal{G}(2,1) \cap \mathcal{H})$ be the cohomology bundle of the monad of the form (6):

$$
\begin{aligned}
& M^{\bullet}: 0 \rightarrow M^{-1} \stackrel{\alpha}{\rightarrow} M^{0} \stackrel{\beta}{\rightarrow} M^{1} \rightarrow 0, \\
& M^{-1}=\mathcal{O}_{\mathbb{P}^{3}}(-2) \oplus V_{2} \otimes \mathcal{O}_{\mathbb{P}^{3}}(-1), M^{0}=\mathcal{O}_{\mathbb{P}^{3}}(-1) \oplus V_{6} \otimes \mathcal{O}_{\mathbb{P}^{3}} \oplus \mathcal{O}_{\mathbb{P}^{3}}(1), \\
& M^{1}=V_{2}^{\prime} \otimes \mathcal{O}_{\mathbb{P}^{3}}(1) \oplus \mathcal{O}_{\mathbb{P}^{3}}(2) .
\end{aligned}
$$

Since the bundle $V_{2} \otimes \mathcal{O}_{\mathbb{P}^{3}}(-1)$ is a uniquely defined subbundle of the bundle $M^{-1}$ (respectively, $V_{2}^{\prime} \otimes \mathcal{O}_{\mathbb{P}^{3}}(1)$ is a uniquely defined quotient bundle of $M^{1}$ ), we obtain a commutative diagram in which $\alpha_{0}$ and $\beta_{0}$ are the induced morphisms:

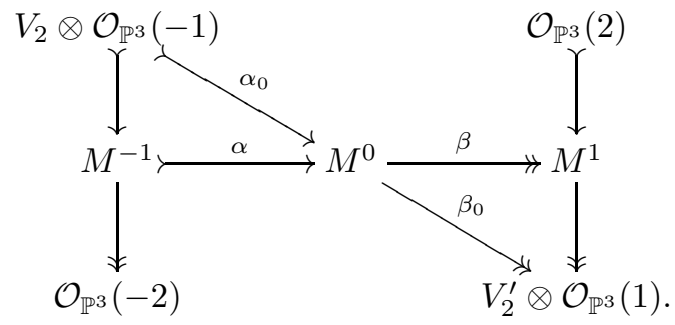


Here the induced monad

$$
0 \rightarrow V_{2} \otimes \mathcal{O}_{\mathbb{P}^{3}}(-1) \stackrel{\alpha_{0}}{\longrightarrow} M^{0} \stackrel{\beta_{0}}{\longrightarrow} V_{2}^{\prime} \otimes \mathcal{O}_{\mathbb{P}^{3}}(1) \rightarrow 0
$$

has the rank 4 cohomology bundle

$$
E=\frac{\operatorname{ker} \beta_{0}}{\operatorname{im} \alpha_{0}}
$$

Mimicking now the argument with diagram (37), we obtain that there exist a subbunle morphism $\sigma: \mathcal{O}_{\mathbb{P}^{3}}(-2) \rightarrow E$ and an epimorphism $\tau: E \rightarrow \mathcal{O}_{\mathbb{P}^{3}}(2)$ which yield the monad the the cohomology bundle $\mathcal{E}$ :

$$
0 \rightarrow \mathcal{O}_{\mathbb{P}^{3}}(-2) \stackrel{\sigma}{\rightarrow} E \stackrel{\tau}{\rightarrow} \mathcal{O}_{\mathbb{P}^{3}}(2) \rightarrow 0, \quad \mathcal{E}=\operatorname{ker} \tau / \operatorname{im} \sigma .
$$

Since there is a uniquely defined (up to a scalar multiple) quotient morphism $M^{0} \rightarrow \mathcal{O}_{\mathbb{P}^{3}}(-1)$, we have a welldefined morphism

$$
\tilde{\alpha}: V_{2} \otimes \mathcal{O}_{\mathbb{P}^{3}}(-1) \stackrel{\alpha_{0}}{\hookrightarrow} M^{0} \rightarrow \mathcal{O}_{\mathbb{P}^{3}}(-1)
$$

and, dually, a well-defined morphism

$$
\tilde{\beta}: \mathcal{O}_{\mathbb{P}^{3}}(1) \hookrightarrow M^{0} \stackrel{\beta_{0}}{\rightarrow} V_{2}^{\prime} \otimes \mathcal{O}_{\mathbb{P}^{3}}(1) .
$$

Assume that both $\tilde{\alpha}$ and $\tilde{\beta}$ are nonzero morphisms. Then a standard diagram chasing shows that, in the monad (132), one can split out a direct summand $\mathcal{O}_{\mathbb{P}^{3}}(-1)$ from $V_{2} \otimes \mathcal{O}_{\mathbb{P}^{3}}(-1)$ and $M^{0}$, respectively, split out a direct summand $\mathcal{O}_{\mathbb{P}^{3}}(1)$ from $M^{0}$ and $V_{2}^{\prime} \otimes \mathcal{O}_{\mathbb{P}^{3}}(1)$, without changing its cohomology bundle $E$. Thus, the monad (132) reduces to a monad

$$
0 \rightarrow \mathcal{O}_{\mathbb{P}^{3}}(-1) \stackrel{\alpha^{\prime}}{\rightarrow} V_{6} \otimes \mathcal{O}_{\mathbb{P}^{3}} \stackrel{\beta^{\prime}}{\rightarrow} \mathcal{O}_{\mathbb{P}^{3}}(1) \rightarrow 0, \quad E=\frac{\operatorname{ker} \beta^{\prime}}{\operatorname{im~} \alpha^{\prime}}
$$

Now by the remark after Lemma 4, $E$ is a rank 4 instanton bundle, so that, by (134) and Lemma 10, $\mathcal{E}$ is the cohomology bundle of the monad (32) for $a=2$ and $k=1$. This means that $\mathcal{E} \in \mathcal{G}(2,1) \cap \mathcal{H}$, contrary to the assumption on $\mathcal{E}$.

We thus may assume that either (a) $\tilde{\alpha}=0, \tilde{\beta} \neq 0$, or (b) $\tilde{\alpha}=\tilde{\beta}=0$. (We omit the case $\tilde{\alpha} \neq 0, \tilde{\beta}=0$, since it is completely similar to the case (a).)

(a) Case $\tilde{\alpha}=0, \tilde{\beta} \neq 0$. We are going to show that this case is impossible.

First, note that, since $\tilde{\beta} \neq 0$, we may as above split out a direct summand $\mathcal{O}_{\mathbb{P}^{3}}(1)$ from the middle term and the righthand term of the monad (132), without changing its cohomology bundle $E$. Thus, this monad reduces to a monad

$$
0 \rightarrow V_{2} \otimes \mathcal{O}_{\mathbb{P}^{3}}(-1) \stackrel{\alpha^{\prime}}{\rightarrow} \mathcal{O}_{\mathbb{P}^{3}}(-1) \oplus V_{6} \otimes \mathcal{O}_{\mathbb{P}^{3}} \stackrel{\beta^{\prime}}{\rightarrow} \mathcal{O}_{\mathbb{P}^{3}}(1) \rightarrow 0, \quad E=\frac{\operatorname{ker} \beta^{\prime}}{\operatorname{im} \alpha^{\prime}}
$$

Next, the condition $\tilde{\alpha}=0$ means that the subbundle morphism $\alpha^{\prime}$ in (132) factors through a subbundle morphism $\alpha^{\prime \prime}$ in the commutative diagram

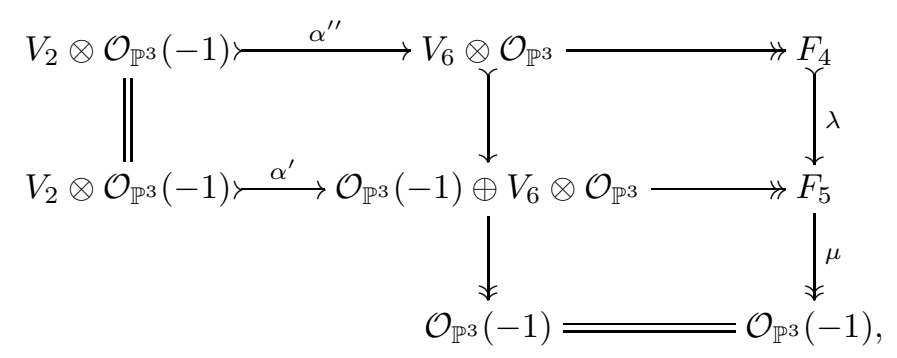

where $F_{4}:=$ coker $\alpha^{\prime \prime}$ and $F_{5}:=$ coker $\alpha^{\prime}$ are vector bundles of rank 4 and 5 , respectively. From this diagram it follows immediately that $\mathcal{O}_{\mathbb{P}^{3}}(-1)$ splits out as a direct summand of $F_{5}$ :

$$
F_{5} \cong \mathcal{O}_{\mathbb{P}^{3}}(-1) \oplus F_{4} .
$$


The monad (138) and the diagram (139) yield a commutative diagram

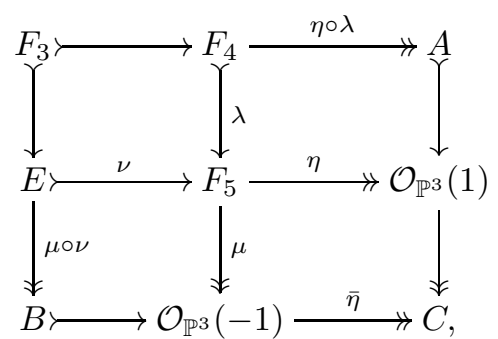

where $F_{3}:=\operatorname{ker}(\eta \circ \lambda), A:=F_{4} / F_{3}, B:=E / F_{3}, C:=\mathcal{O}_{\mathbb{P}^{3}}(1) / A$. Here $A \neq 0$, since otherwise $C \simeq \mathcal{O}_{\mathbb{P}^{3}}(1)$, and then $\bar{\eta}$ is not surjective, contrary to (141). Hence, $C$ is a torsion sheaf, and $A, B$ and $F_{3}$ are torsion free sheaves of rank 1, 1 and 4, respectively. Therefore, the diagram (141) implies that $c_{1}\left(F_{4}\right)-c_{1}(E)=2 c_{1}\left(\mathcal{O}_{\mathbb{P}^{3}}(1)\right)$. On the other hand, in view of (140) we have a well-defined injective morphism $\rho: E \stackrel{\nu}{\rightarrow} F_{5} \stackrel{p r_{2}}{\rightarrow} F_{4}$ such that, by the Snake Lemma, $Q:=\operatorname{coker} \rho \cong A / B$ is a torsion sheaf. In addition, by the above equality, $c_{1}(Q)=2 c_{1}\left(\mathcal{O}_{\mathbb{P}^{3}}(1)\right) \neq 0$, i. e., $Q \neq 0$. However, (141) and the Snake Lemma yield a commutative diagram

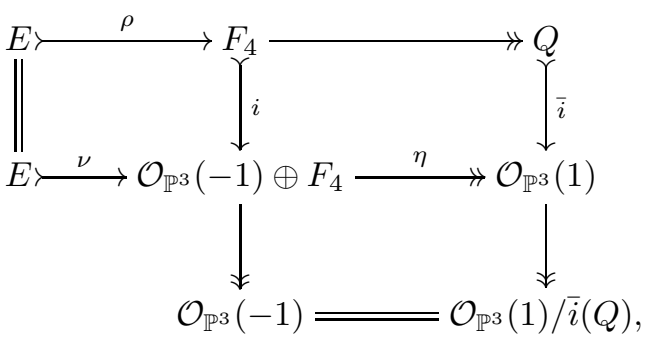

where $i$ is the inclusion of the direct summand and $\bar{i}$ is the induced morphism. But the torsion sheaf $Q$ is not a subsheaf of $\mathcal{O}_{\mathbb{P}^{3}}(1)$, and we obtain a contradiction, as claimed.

Summarizing the above arguments, we see that the bundle $[\mathcal{E}] \in \mathcal{H}$ is the cohomology $\mathcal{H}^{0}\left(M^{\bullet}\right)$ of a $\operatorname{monad} M^{\bullet}$ of the form ([6) satisfying the condition (a): $(\tilde{\alpha}, \tilde{\beta}) \neq(0,0)$, then $M^{\bullet}$ is reducible to a monad of the form (5), i. e. $[\mathcal{E}] \in \mathcal{H} \cap \mathcal{G}(2,1)$. Thus, denoting

$$
\mathcal{H}_{0}:=\left\{[\mathcal{E}] \in \mathcal{H} \mid \mathcal{E}=\mathcal{H}^{0}\left(M^{\bullet}\right) \text {, where } M^{\bullet} \text { satisfies the condition (b): } \quad \tilde{\alpha}=\tilde{\beta}=0\right\},
$$

we obtain

$$
\mathcal{H} \backslash(\mathcal{H} \cap \mathcal{G}(2,1)) \subset \mathcal{H}_{0} .
$$

We thus proceed to the study of the case $\tilde{\alpha}=\tilde{\beta}=0$.

(b) Case $\tilde{\alpha}=\tilde{\beta}=0$.

First, consider the commutative diagram

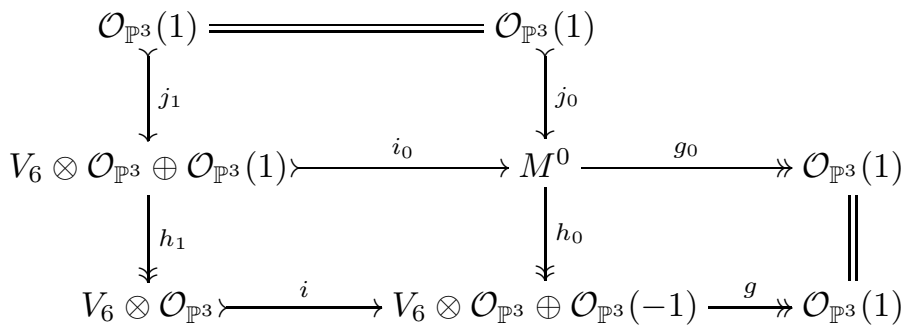

and the exact triples following from (132) and (133)

$$
\begin{gathered}
0 \rightarrow V_{2} \otimes \mathcal{O}_{\mathbb{P}^{3}}(-1) \stackrel{\alpha_{0}}{\rightarrow} M^{0} \stackrel{c_{0}}{\rightarrow} C_{0} \rightarrow 0, \\
0 \rightarrow E \stackrel{d_{0}}{\longrightarrow} C_{0} \stackrel{e_{0}}{\longrightarrow} V_{2}^{\prime} \otimes \mathcal{O}_{\mathbb{P}^{3}}(1) \rightarrow 0, \quad C_{0}:=\text { coker } \alpha_{0}, \quad \beta_{0}=e_{0} \circ c_{0} .
\end{gathered}
$$

The condition $\tilde{\alpha}=0$ implies that there exists a subbundle morphism $0 \rightarrow V_{2} \otimes \mathcal{O}_{\mathbb{P}^{3}}(-1) \stackrel{\alpha_{1}}{\longrightarrow} V_{6} \otimes \mathcal{O}_{\mathbb{P}^{3}} \oplus \mathcal{O}_{\mathbb{P}^{3}}(1)$ such that

$$
\alpha_{0}=i_{0} \circ \alpha_{1} .
$$


Setting $C:=\operatorname{coker}\left(h_{0} \circ \alpha_{0}\right), C_{1}:=\operatorname{coker} \alpha_{1}, \alpha_{2}:=h_{1} \circ \alpha_{1}, C_{2}:=$ coker $\alpha_{2}$, we obtain from (145)-(146) and (148) an induced commutative diagram

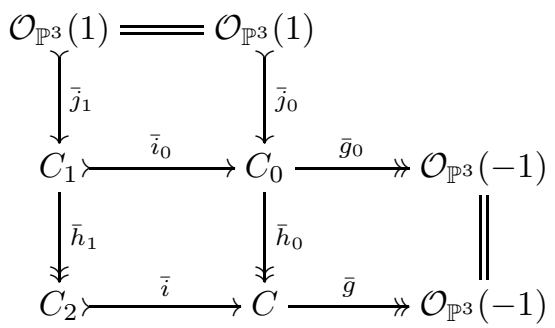

and an exact triple

$$
0 \rightarrow V_{2} \otimes \mathcal{O}_{\mathbb{P}^{3}}(-1) \stackrel{\alpha_{2}}{\longrightarrow} V_{6} \otimes \mathcal{O}_{\mathbb{P}^{3}} \stackrel{c_{2}}{\longrightarrow} C_{2} \rightarrow 0 .
$$

From the condition $\tilde{\beta}=0$ and diagram chasing it follows that there exists an injective morphism $j: \mathcal{O}_{\mathbb{P}^{3}}(1) \rightarrow E$ such that $\bar{j}_{0}=d_{0} \circ j$. From this relation and (147), (149) and (150) by diagram chasing we obtain the folowing data:

1) an exact triple

$$
0 \rightarrow \mathcal{O}_{\mathbb{P}^{3}}(1) \stackrel{j}{\rightarrow} E \stackrel{h}{\rightarrow} E_{3} \rightarrow 0, \quad E_{3}:=\text { coker } j
$$

2) a commutative diagram

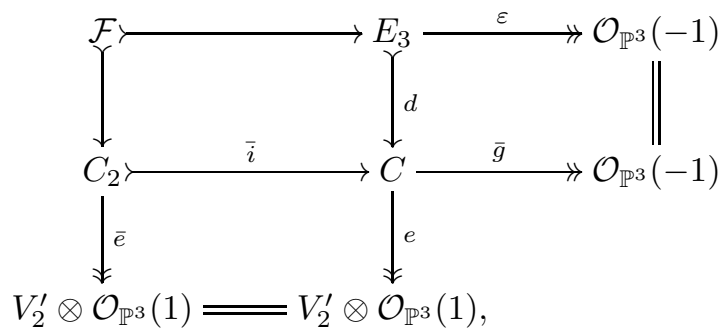

where $d$ and $e$ are the induced morphisms, $\varepsilon:=d \circ \bar{g}, \bar{e}:=e \circ \bar{i}$, 3) a sheaf

$$
\mathcal{F}:=\operatorname{ker} \varepsilon
$$

and a left-exact complex

$$
\begin{aligned}
& K^{\bullet}: 0 \rightarrow K^{-1} \stackrel{\alpha_{2}}{\longrightarrow} K^{0} \stackrel{\beta_{2}}{\longrightarrow} K^{1} \rightarrow 0, \quad \beta_{2}:=\bar{e} \circ c_{2}, \\
& K^{-1}=V_{2} \otimes \mathcal{O}_{\mathbb{P}^{3}}(-1), \quad K^{0}=V_{6} \otimes \mathcal{O}_{\mathbb{P}^{3}}, \quad K^{1}=V_{2}^{\prime} \otimes \mathcal{O}_{\mathbb{P}^{3}}(1),
\end{aligned}
$$

such that

$$
\mathcal{H}^{0}\left(K^{\bullet}\right)=\mathcal{F}, \quad \mathcal{H}^{1}\left(K^{\bullet}\right)=\text { coker } \varepsilon .
$$

From (134), (154) and the vanishing of $\operatorname{Hom}\left(\mathcal{O}_{\mathbb{P}^{3}}(1), \mathcal{O}_{\mathbb{P}^{3}}(-2)\right)$ follows the commutative diagram

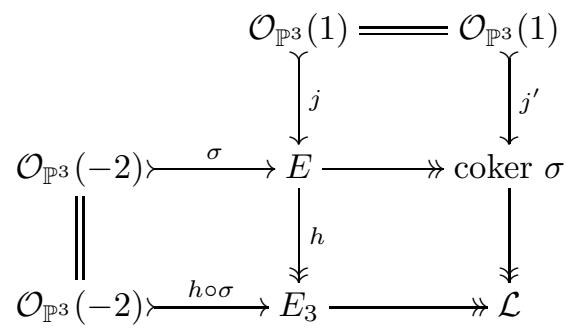

where $\mathcal{L}:=\operatorname{coker}(h \circ \sigma)$ and $j^{\prime}$ is an induced morphism which is nonzero, hence injective, since coker $\sigma$ is locally free by the exact triple $0 \rightarrow \mathcal{E} \rightarrow$ coker $\sigma \rightarrow \mathcal{O}_{\mathbb{P}^{3}}(2) \rightarrow 0$ following from (134). Since $\mathcal{E}$ is stable by assumption, so 
that $h^{0}(\mathcal{E}(-1))=0$ (see [34]), the last triple and (156) yield a commutative diagram

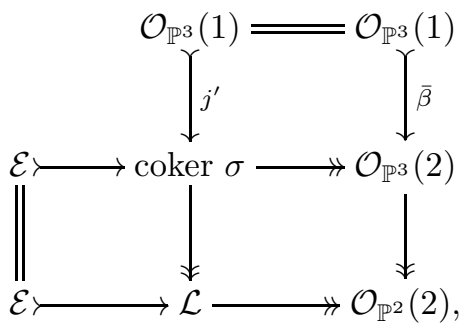

where $\mathbb{P}^{2}=\operatorname{Supp}(\operatorname{coker} \bar{\beta})$ is a projective plane in $\mathbb{P}^{3}$. Note that, in this diagram, $\bar{\beta}$ is the composition $\bar{\beta}$ : $\mathcal{O}_{\mathbb{P}^{3}}(1) \stackrel{\text { can }}{\longrightarrow} M^{0} \stackrel{\beta}{\rightarrow} M^{1}$, and $\operatorname{im} \bar{\beta} \hookrightarrow \mathcal{O}_{\mathbb{P}^{3}}(2)$ since $\tilde{\beta}=0$. Thus, $\mathbb{P}^{2}$ is uniquely defined by the morphism $\beta$ in the monad $M^{\bullet}$. In a similar way, since $\tilde{\alpha}=0$, the morphism $\alpha$ in $M^{\bullet}$ uniquely defines a morphism $\bar{\alpha}: \mathcal{O}_{\mathbb{P}^{3}}(-2) \rightarrow \mathcal{O}_{\mathbb{P}^{3}}(-1)$, hence a projective plane $\mathbb{P}_{0}^{2}=\operatorname{Supp}(\operatorname{coker} \bar{\alpha})$. For these two planes we will use the notation

$$
\mathbb{P}^{2}=\mathbb{P}^{2}\left(M^{\bullet}, \beta\right), \mathbb{P}_{0}^{2}=\mathbb{P}^{2}\left(M^{\bullet}, \alpha\right) .
$$

Consider the lower horizontal triple in (157):

$$
0 \rightarrow \mathcal{E} \stackrel{\theta}{\rightarrow} \mathcal{L} \stackrel{\gamma}{\rightarrow} \mathcal{O}_{\mathbb{P}^{2}}(2) \rightarrow 0, \quad \mathbb{P}^{2}=\mathbb{P}^{2}\left(M^{\bullet}, \beta\right) .
$$

Lemma 22. The sheaf $\mathcal{L}$ in (159) is a stable reflexive rank 2 sheaf on $\mathbb{P}^{3},[\mathcal{L}] \in \mathcal{R}(1,4,6)$.

Proof. First, show that the triple (159) doesn't split. Indeed, otherwise, the lower horizontal triple in (156) extends to a commutative push-out diagram

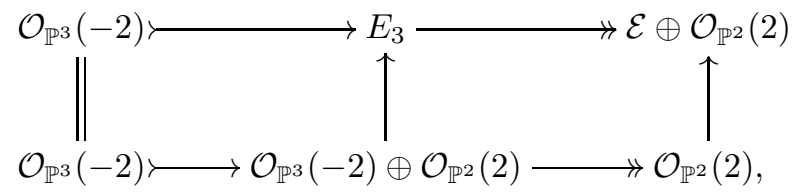

where the lower triple splits since $\operatorname{Ext}^{1}\left(\mathcal{O}_{\mathbb{P}^{2}}(2), \mathcal{O}_{\mathbb{P}^{3}}(-2)\right)=0$. This yields a nonzero morphism $\delta: \mathcal{O}_{\mathbb{P}^{2}}(2) \rightarrow E_{3}$ which being composed with the morphism $\varepsilon$ in (153) is the zero morphism $\mathcal{O}_{\mathbb{P}^{2}}(2) \rightarrow \mathcal{O}_{\mathbb{P}^{3}}(-1)$. Hence, in (153), $\delta$ factors through a nonzero morphism

$$
\delta^{\prime}: \mathcal{O}_{\mathbb{P}^{2}}(2) \rightarrow \mathcal{F}
$$

On the other hand, (154) and (155) yield an exact triple $0 \rightarrow \mathcal{O}_{\mathbb{P}^{3}}(-2) \rightarrow$ ker $\beta_{2} \rightarrow \mathcal{F} \rightarrow 0$ which, together with (161), extends to a push-out diagram similar to (160):

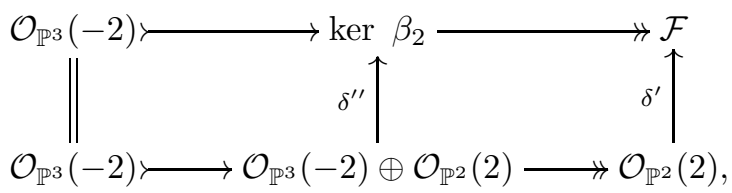

where $\left.\delta^{\prime \prime}\right|_{\mathcal{O}^{2}(2)}$ is nonzero. However, this is impossible since ker $\beta_{2}$ by definition is torsion free as a subsheaf of a locally free sheaf $V_{6} \otimes \mathcal{O}_{\mathbb{P}^{3}}$.

Next, since $\mathcal{E} \cong \mathcal{E}^{\vee}$ is locally free and $\mathcal{E} x t^{1}\left(\mathcal{O}_{\mathbb{P}^{2}}(2), \mathcal{O}_{\mathbb{P}^{3}}\right)=\mathcal{O}_{\mathbb{P}^{2}}(-1)$, then, applying the functor $\mathcal{E} x t^{\bullet}\left(-, \mathcal{O}_{\mathbb{P}^{3}}\right)$ to the triple (159) we have an exact sequence

$$
0 \rightarrow \mathcal{L}^{\vee} \stackrel{\theta^{\vee}}{\rightarrow} \mathcal{E} \rightarrow \mathcal{O}_{\mathbb{P}^{2}}(-1) \stackrel{\varphi}{\rightarrow}{ }^{1} \mathcal{L} \rightarrow 0, \quad{ }^{1} \mathcal{L}:=\mathcal{E} x t^{1}\left(\mathcal{L}, \mathcal{O}_{\mathbb{P}^{3}}\right) .
$$

Let $d=\operatorname{dim}\left({ }^{1} \mathcal{L}\right)$. Consider the three possible cases: (a) $d=2$, (b) $d=1$, and (c) $d=0$. We will show that the cases (a) and (b) lead to a contradition.

(a) $d=2$. In this case $\operatorname{dim} \operatorname{Sing} \mathcal{L}=2$, i. e. the torsion subsheaf $\mathcal{T} \operatorname{ors}(\mathcal{L})$ of $\mathcal{L}$ has dimension 2. This necessarily implies that the composition $\mathcal{T} \operatorname{ors}(\mathcal{L}) \hookrightarrow \mathcal{L} \stackrel{\gamma}{\rightarrow} \mathcal{O}_{\mathbb{P}^{2}}(2)$ is an isomorphism giving the splitting of the triple (159), contrary to the above.

(b) $d=1$. In this case ${ }^{1} \mathcal{L}=\mathcal{O}_{Z}(-1)$ for $Z$ a subscheme of $\mathbb{P}^{2}$, of dimension $\operatorname{dim} Z=1$. Hence $\operatorname{ker} \varphi \hookrightarrow \mathcal{O}_{\mathbb{P}^{2}}(-1-k)$ for some $k \geq 2$. By (163) the sheaf $\operatorname{ker} \varphi$ is the quotient of $\mathcal{E}$, it follows that $h^{0}\left(\mathcal{E}_{\mathbb{P}^{2}}(-1-k)\right) \neq 0, k \geq 1$, and so 
$h^{0}\left(\mathcal{E}_{\mathbb{P}^{2}}(-2)\right) \neq 0$. On the other hand, since $\mathcal{E}$ is the cohomology of (130), by [21, Table 5.3, case 5(2.ii)] it has the spectrum $\operatorname{Sp}(\mathcal{E})=(-1,0,0,0,1)$, and then it follows that

$$
h^{1}(\mathcal{E}(-3))=0, \quad h^{1}(\mathcal{E}(-2))=1 .
$$

Thus, by the first equality in (164), the inequality $h^{0}\left(\mathcal{E}_{\mathbb{P}^{2}}(-2)\right) \neq 0$ contradicts to the cohomology sequence of the exact triple

$$
0 \rightarrow \mathcal{E}(-3) \rightarrow \mathcal{E}(-2) \rightarrow \mathcal{E}_{\mathbb{P}^{2}}(-2) \rightarrow 0
$$

as $h^{0}(\mathcal{E}(-2))=0$ by the stability of $E$. Note also that we have proved here the equality

$$
H^{0}\left(\mathcal{E}_{\mathbb{P}^{2}}(-2)\right)=0, \quad \forall \mathbb{P}^{2} \subset \mathbb{P}^{3} .
$$

(c) $d=1$. In this case ${ }^{1} \mathcal{L}=\mathcal{O}_{Z}(-1)$ for $Z$ a subscheme of $\mathbb{P}^{2}$ of dimension $\operatorname{dim} Z=0$, and the sequence $0 \rightarrow \mathcal{L}^{\vee} \stackrel{\theta^{\vee}}{\longrightarrow} \mathcal{E} \rightarrow \mathcal{I}_{Z, \mathbb{P}^{2}}(-1) \rightarrow 0$, and $Z=(s)_{0}, 0 \neq s \in H^{0}\left(\left.\mathcal{E}(-1)\right|_{\mathbb{P}^{2}}\right)$. Since $\operatorname{dim} Z=0$, it follows that $\mathcal{E} x t^{1}\left(\mathcal{I}_{Z, \mathbb{P}^{2}}(-1), \mathcal{O}_{\mathbb{P}^{3}}\right)=\mathcal{E} x t^{1}\left(\mathcal{O}_{\mathbb{P}^{2}}(-1), \mathcal{O}_{\mathbb{P}^{3}}\right)=\mathcal{O}_{\mathbb{P}^{2}}(2)$. Thus, applying the functor $\mathcal{E} x t^{\bullet}\left(-, \mathcal{O}_{\mathbb{P}^{3}}\right)$ to the last triple, since $\mathcal{E} \cong \mathcal{E}^{\vee}$ we obtain an exact triple $0 \rightarrow \mathcal{E} \stackrel{\theta^{\vee \vee}}{\longrightarrow} \mathcal{L}^{\vee \vee} \stackrel{\gamma}{\rightarrow} \mathcal{O}_{\mathbb{P}^{2}}(2) \rightarrow 0$. Comparing this triple with (159) and taking into account that, by construction, the composition $\mathcal{E} \stackrel{\theta}{\rightarrow} \mathcal{L} \stackrel{\text { can }}{\longrightarrow} \mathcal{L}^{\vee \vee}$ coincides with $\theta^{\vee \vee}$ we obtain that $\mathcal{L} \stackrel{\text { can }}{\longrightarrow} \mathcal{L}^{\vee \vee}$ is an isomorphism, i. e. $\mathcal{L}$ is reflexive.

Next, as $c_{t}(\mathcal{E})=1+5 t^{2}$, formulas for Chern classes of $\mathcal{L}$ follow from (159). In particular, $\mathcal{L}^{\vee} \cong \mathcal{L}(-1)$ has $c_{1}(\mathcal{L}(-1))=-1$, and since $h^{0}(\mathcal{E})=0$, it follows that

$$
h^{0}(\mathcal{L}(-1))=0 .
$$

Thus, $\mathcal{L}$ is stable by [20, Lemma 3.1]. Lemma is proved.

We now proceed to the more close study of the sheaf $\mathcal{F}$. Consider the upper horizontal triple of the diagram (152) which extends to an exact sequence:

$$
0 \rightarrow \mathcal{F} \rightarrow E_{3} \stackrel{\varepsilon}{\rightarrow} \mathcal{O}_{\mathbb{P}^{3}}(-1) \rightarrow \mathcal{O}_{\bar{Y}}(-1) \rightarrow 0, \quad \bar{Y} \subset \mathbb{P}^{3} .
$$

Lemma 23. The sheaf $\mathcal{F}$ defined in $(153)$ is a reflexive rank 2 sheaf on $\mathbb{P}^{3}$ fitting in an exact triple

$$
0 \rightarrow \mathcal{F} \stackrel{\zeta}{\rightarrow} \mathcal{L} \rightarrow \mathcal{I}_{\bar{Y}, \mathbb{P}_{0}^{2}}(-1) \rightarrow 0
$$

and in its dual

$$
0 \rightarrow \mathcal{L}(-1) \rightarrow \mathcal{F} \stackrel{\rho}{\rightarrow} \mathcal{I}_{\bar{Z}, \mathbb{P}_{0}^{2}}(2) \rightarrow 0
$$

where $\mathbb{P}_{0}^{2}=\mathbb{P}^{2}\left(M^{\bullet}, \alpha\right), \bar{Y}, \bar{Z} \subset \mathbb{P}_{0}^{2}, \operatorname{dim} \bar{Y} \leq 0, \operatorname{dim} \bar{Z} \leq 0$, and

$$
\ell(\bar{Y})+\ell(\bar{Z})=6
$$

Chern classes of $\mathcal{F}$ are $c_{1}(\mathcal{F})=0, c_{2}(\mathcal{F})=2,0 \leq c_{3}(\mathcal{F})=2 \ell(\bar{Y}) \leq 12$.

Proof. We first show that $\mathrm{rk} \mathcal{F}=2$. Indeed, if $\varepsilon$ in (168) is the zero morphism, then the diagram (152) and the Snake Lemma yield an epimorphism $V_{2}^{\prime} \otimes \mathcal{O}_{\mathbb{P}^{3}}(1) \rightarrow \mathcal{O}_{\mathbb{P}^{3}}(-1)$ which is impossible. Hence, $\varepsilon \neq 0$ and (153) implies that $\mathrm{rk} \mathcal{F}=2$ and, moreover, that $\bar{Y} \varsubsetneqq \mathbb{P}^{3}$, i. e., $\bar{Y}$ is a proper subscheme of $\mathbb{P}^{3}$. Note also that, by (132) and (133), $c_{1}(E)=0$, hence $c_{1}\left(E_{3}\right)=-1$ in view of (151). Thus, (168) implies that $c_{1}(\mathcal{F})=c_{1}\left(\mathcal{O}_{\bar{Y}}(-1)\right) \geq 0$.

Next, consider the lower exact triple in (156):

$$
0 \rightarrow \mathcal{O}_{\mathbb{P}^{3}}(-2) \stackrel{h \circ \sigma}{\longrightarrow} E_{3} \rightarrow \mathcal{L} \rightarrow 0 .
$$

If the composition $f:=\varepsilon \circ h \circ \sigma$ is zero, then (168) and (172) imply that there exist injective morphisms $\mathcal{O}_{\mathbb{P}^{3}}(-2) \stackrel{f_{1}}{\longmapsto} \mathcal{F}$ and $\operatorname{coker}\left(f_{1}\right) \stackrel{f_{2}}{\longmapsto} \mathcal{L}$. Since $\mathrm{rk} \mathcal{F}=2, c_{1}(\mathcal{F}) \geq 0$ and $\mathcal{L}$ is reflexive by Lemma 22, it follows that coker $\left(f_{1}\right)$ is a rank 1 torsion free sheaf with $c_{1}\left(\operatorname{coker}\left(f_{1}\right)\right) \geq 2$. Thus, the injectivity of $f_{2}$ shows that $h^{0}(\mathcal{L}(-2)) \neq 0$, contrary to the stability of $\mathcal{L}$ (see Lemma 22). It follows that $f \neq 0$, so that (168) and (172) extend to a comutative diagram

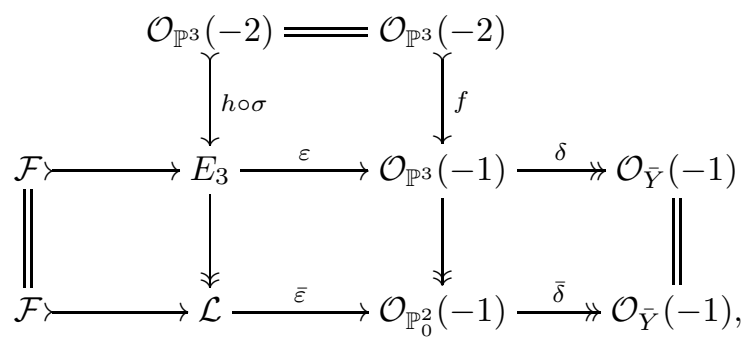


where $\mathbb{P}_{0}^{2}$ is some projective plane in $\mathbb{P}^{3}$. If $\bar{\delta}$ is an isomorphism, then $\operatorname{coker}(\varepsilon) \simeq \mathcal{O}_{\mathbb{P}_{0}^{2}}(-1)$, so that the diagram (152) and the Snake Lemma yield an epimorphism $V_{2}^{\prime} \otimes \mathcal{O}_{\mathbb{P}^{3}}(1) \rightarrow \mathcal{O}_{\mathbb{P}_{0}^{2}}(-1)$ which is impossible. Hence, $\bar{Y} \varsubsetneqq \mathbb{P}_{0}^{2}$, i. e., $\bar{Y}$ is a proper subscheme of $\mathbb{P}_{0}^{2}, \operatorname{dim} \bar{Y} \leq 1$, and (173) yields an exact triple (169).

Show that the case $\operatorname{dim} \bar{Y}=1$ is impossible. Indeed, in this case $\bar{Y}$ contains a divisor $D \subset \mathbb{P}_{0}^{2}$ of degree $k \geq 1$ as a subscheme, and this yields an epimorphim $\mathcal{O}_{\bar{Y}}(-1) \stackrel{b}{\rightarrow} \mathcal{O}_{D}(-1)$. On the other hand, the middle horizontal exact sequence in (173), together with diagram (152) and the Snake Lemma, yield an epimorphism $V_{2}^{\prime} \otimes \mathcal{O}_{\mathbb{P}^{3}}(1) \rightarrow \mathcal{O}_{\bar{Y}}(-1)$. This epimorphism composed with the above epimorphism $b$ gives an epimorphism $V_{2}^{\prime} \otimes \mathcal{O}_{\mathbb{P}^{3}}(1) \rightarrow \mathcal{O}_{D}(-1)$ which is impossible, since $h^{0}\left(\mathcal{O}_{D}(-2)\right)=0$, as follows from the cohomology of the exact triple $0 \rightarrow \mathcal{O}_{\mathbb{P}_{0}^{2}}(-2-k) \rightarrow \mathcal{O}_{\mathbb{P}_{0}^{2}}(-k) \rightarrow \mathcal{O}_{D}(-2) \rightarrow 0$.

Hence, $\operatorname{dim} \bar{Y} \leq 0$ and therefore, denoting ${ }^{i} \mathcal{I}:=\mathcal{E} x t^{i}\left(\mathcal{I}_{\bar{Y}, \mathbb{P}_{0}^{2}}(-1), \mathcal{O}_{\mathbb{P}^{3}}\right), i \geq 1$, we obtain ${ }^{1} \mathcal{I}=\mathcal{O}_{\mathbb{P}^{2}}(2), \operatorname{dim}^{2} \mathcal{I} \leq$ $0,{ }^{3} \mathcal{I}=0$. Besides, set ${ }^{i} \mathcal{F}:=\mathcal{E} x t^{i}\left(\mathcal{F}, \mathcal{O}_{\mathbb{P}^{3}}\right),{ }^{i} \mathcal{L}:=\mathcal{E} x t^{i}\left(\mathcal{L}, \mathcal{O}_{\mathbb{P}^{3}}\right), i \geq 1$, and remark that, for the reflexive sheaf $\mathcal{L}, \operatorname{dim}{ }^{1} \mathcal{L}=0,{ }^{i} \mathcal{L}=0, i=2,3$ (see [20, proof of Thm. 2.5]). Now, applying to (169) the functor $\mathcal{E} x t^{\bullet}\left(-, \mathcal{O}_{\mathbb{P}^{3}}\right)$ and using the above relations we obtain the equalities ${ }^{i} \mathcal{F}=0, i=2,3$, and an exact sequence $0 \rightarrow \mathcal{L}^{\vee} \stackrel{\zeta^{\vee}}{\rightarrow} \mathcal{F}^{\vee} \rightarrow \mathcal{O}_{\mathbb{P}^{2}}(2) \stackrel{\mu}{\rightarrow}{ }^{1} \mathcal{L} \rightarrow{ }^{1} \mathcal{F} \rightarrow{ }^{2} \mathcal{I} \rightarrow 0$, wherefrom $\operatorname{dim}^{1} \mathcal{F} \leq 0$ and ker $\mu \simeq \mathcal{I}_{Z, \mathbb{P}_{0}^{2}}(2)$ for some subscheme $Z$ of $\mathbb{P}_{0}^{2}$, of dimension $\operatorname{dim} Z \leq 0$. We thus obtain an exact triple $0 \rightarrow \mathcal{L}^{\vee} \stackrel{\zeta^{\vee}}{\longrightarrow} \mathcal{F}^{\vee} \rightarrow \mathcal{I}_{Z, \mathbb{P}_{0}^{2}}(2) \rightarrow 0$ and the relation $\mathcal{E} x t^{1}\left(\mathcal{I}_{Y, \mathbb{P}_{0}^{2}}(2), \mathcal{O}_{\mathbb{P}^{3}}\right)=\mathcal{O}_{\mathbb{P}^{2}}(-1)$. Next, applying to the last triple the functor $\mathcal{E} x t^{\bullet}\left(-, \mathcal{O}_{\mathbb{P}^{3}}\right)$ yields an exact sequence $0 \rightarrow \mathcal{F}^{\vee \vee} \stackrel{\zeta^{\vee \vee}}{\longrightarrow} \mathcal{L}^{\vee \vee} \rightarrow \mathcal{O}_{\mathbb{P}^{2}}(-1) \stackrel{\nu}{\rightarrow} \mathcal{E} x t^{1}\left(\mathcal{F}^{\vee}, \mathcal{O}_{\mathbb{P}^{3}}\right)$. By 20, Cor. 1.2] $\mathcal{F}^{\vee}$ is a reflexive rank 2 sheaf, hence $\operatorname{dim} \mathcal{E} x t^{1}\left(\mathcal{F}^{\vee}, \mathcal{O}_{\mathbb{P}^{3}}\right) \leq 0$ by [20, Rem. 2.7.1], and therefore ker $\nu \simeq \mathcal{I}_{W, \mathbb{P}_{0}^{2}}(-1)$ for some subscheme $W$ of $\mathbb{P}_{0}^{2}$, of dimension $\operatorname{dim} W \leq 0$. Thus the last sequence leads to an exact triple $0 \rightarrow \mathcal{F}^{\vee \vee} \stackrel{\zeta^{\vee \vee}}{\longrightarrow} \mathcal{L}^{\vee \vee} \rightarrow \mathcal{I}_{W, \mathbb{P}_{0}^{2}}(-1) \rightarrow 0$ which together with (169) fits in a commutative diagram

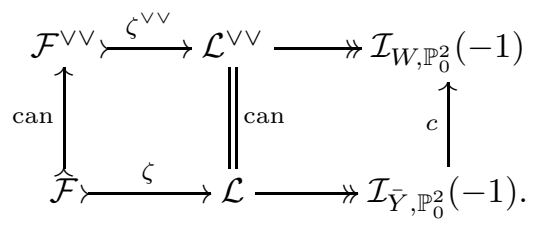

Besides, the above stated relations ${ }^{i} \mathcal{F}=0, i=2,3, \operatorname{dim}{ }^{1} \mathcal{F} \leq 0$ show that the sheaf $\mathcal{F}$ is locally free outside the set of dimension $\leq 0$, and this shows that the sheaf $\kappa=\operatorname{coker}\left(\mathcal{F} \stackrel{\text { can }}{\longrightarrow} \mathcal{F}^{\vee \vee}\right)$ has dimension $\leq 0$ and by the Snake Lemma $\kappa$ is a subsheaf of ker $c$. However, the sheaf has no subsheaves of dimension 0 . Hence, $\kappa=0$ and $\mathcal{F} \stackrel{\text { can }}{\longrightarrow} \mathcal{F}^{\vee \vee}$ is an isomorphism, i. e. $\mathcal{F}$ is reflexive. A standard computation with the triple (169) yields the values of Chern classes of $\mathcal{F}$, The triple (170) and the equality (171) are obtained by applying to (169) the functor $\mathcal{E} x t^{\bullet}\left(-, \mathcal{O}_{\mathbb{P}^{3}}\right)$ and using formulas for Chern classes of $\mathcal{F}$ and $\mathcal{L}$. The inequality $0 \leq c_{3}(\mathcal{F}) \leq 12$ follows from (171).

Lemma 24. The projective planes $\mathbb{P}^{2}$ and $\mathbb{P}_{0}^{2}$ defined in (158) coincide.

Proof. The middle horizontal triple $0 \rightarrow \mathcal{E} \rightarrow$ coker $\sigma \rightarrow \mathcal{O}_{\mathbb{P}^{3}}(2) \rightarrow 0$ in (157) as an extension is defined by a nonzero element in $\operatorname{Ext}^{1}\left(\mathcal{E}, \mathcal{O}_{\mathbb{P}^{2}}(2)\right) \simeq H^{1}(\mathcal{E}(-2))$. Since $h^{1}(\mathcal{E}(-2))=1$ by (164), it follows that the sheaf coker $\sigma$ is defined by $\mathcal{E}$ uniquely up to an isomorphism. Since $h^{0}(\mathcal{L}(-1))=0$ as $\mathcal{L}$ is stable by Lemma 22, the twisted by $\mathcal{O}_{\mathbb{P}^{3}}(-1)$ middle vertical triple $0 \rightarrow \mathcal{O}_{\mathbb{P}^{3}} \rightarrow \operatorname{coker} \sigma(-1) \rightarrow \mathcal{L}(-1) \rightarrow 0$ in (157) shows that $h^{0}(\operatorname{coker} \sigma(-1))=1$. Hence, $\mathcal{L}=\mathcal{L}\left(M^{\bullet}\right)$ is uniquely up to an isomorphism defined by coker $\sigma$ (and therefore by $\mathcal{E}$ ) as

$$
\mathcal{L}\left(M^{\bullet}\right)=\left(\operatorname{coker} \sigma(-1) / \mathcal{O}_{\mathbb{P}^{3}}\right)(1) .
$$

Then the lower horizontal triple in (157) shows that the plane $\mathbb{P}^{2}=\mathbb{P}^{2}\left(M^{\bullet}, \beta\right)$ is determined uniquely by $\mathcal{E}$ as

$$
\mathbb{P}^{2}\left(M^{\bullet}, \beta\right)=\operatorname{Supp}\left(\mathcal{L}\left(M^{\bullet}\right) / \mathcal{E}\right) .
$$

Next, it follows from (158) that

$$
\mathbb{P}^{2}\left(M^{\bullet}, \alpha\right)=\mathbb{P}^{2}\left(M^{\bullet \vee}, \beta^{\vee}\right), \quad \text { resp., } \quad \mathbb{P}^{2}\left(M^{\bullet}, \beta\right)=\mathbb{P}^{2}\left(M^{\bullet \vee}, \alpha^{\vee}\right),
$$

where $M^{\bullet \vee}: 0 \rightarrow\left(M^{1}\right)^{\vee} \stackrel{\beta^{\vee}}{\longrightarrow}\left(M^{0}\right)^{\vee} \stackrel{\alpha^{\vee}}{\longrightarrow}\left(M^{-1}\right)^{\vee} \rightarrow 0$ is the monad dual to $M^{\bullet}$. The monad $M^{\bullet} \vee$ defines the monad dual to (134): $0 \rightarrow \mathcal{O}_{\mathbb{P}^{3}}(-2) \stackrel{\tau^{\vee}}{\rightarrow} E \stackrel{\sigma^{\vee}}{\rightarrow} \mathcal{O}_{\mathbb{P}^{3}}(2) \rightarrow 0$ with $\mathcal{E}^{\vee}=\operatorname{ker}\left(\sigma^{\vee}\right) / \operatorname{im}\left(\tau^{\vee}\right)$, and the argument dual to the above yields the formulas dual to (174) and (175): $\mathbb{P}^{2}\left(M^{\bullet \vee}, \beta^{\vee}\right)=\operatorname{Supp}\left(\mathcal{L}\left(M^{\bullet \vee}\right) / \mathcal{E}^{\vee}\right), \mathcal{L}\left(M^{\bullet \vee}\right)=$ $\left(\operatorname{coker}\left(\tau^{\vee}\right)(-1) / \mathcal{O}_{\mathbb{P}^{3}}\right)(1)$. Since $\mathcal{E}^{\vee} \simeq \mathcal{E}$ these formulas mean in view of (176) that the plane $\mathbb{P}_{0}^{2}=\mathbb{P}^{2}\left(M^{\bullet}, \alpha\right)$ is uniquely defined by $\mathcal{E}$ via the same construction as above, hence it coincides with $\mathbb{P}^{2}=\mathbb{P}^{2}\left(M^{\bullet}, \beta\right)$. 
Let $\mathcal{F} \in \mathcal{R}(0,2,2 k)$ be the reflexive sheaf defined in (153), where $0 \leq k \leq 6$ by Lemma 23 , i. e.,

$$
[\mathcal{F}] \in \bigsqcup_{0 \leq k \leq 6} \mathcal{R}_{k}, \quad \mathcal{R}_{k}:=\mathcal{R}(0,2,2 k) .
$$

Formulas (143), (144) and Lemmas 22, 23 and 24 yield

Proposition 25. There is an inclusion

$$
\mathcal{H} \backslash(\mathcal{H} \cap \mathcal{G}(2,1)) \subset \bigsqcup_{0 \leq k \leq 6} \mathcal{H}_{k}, \quad \text { where }
$$

$$
\mathcal{H}_{k}=\left\{[\mathcal{E}] \in \mathcal{B}(5) \mid \mathcal{E} \text { is obtained from } \mathcal{F} \text {, where }[\mathcal{F}] \in \mathcal{R}_{k},\right.
$$

by the two subsequent elementary transformations (180) below\},

$\quad 0 \rightarrow \mathcal{E} \rightarrow \mathcal{L} \stackrel{\gamma}{\rightarrow} \mathcal{O}_{\mathbb{P}^{2}}(2) \rightarrow 0, \quad \quad$ (step 2$)$

where $\mathbb{P}^{2}$ is some plane in $\mathbb{P}^{3}, \bar{Z} \subset \mathbb{P}^{2}, \operatorname{dim} \bar{Z} \leq 0, \ell(\bar{Z})=6-k$, and $\mathcal{L}$ is a stable reflexive sheaf from $\mathcal{R}(1,4,6)$.

\section{Geometric properties of sheaves $\mathcal{F}$ and moduli of Cohomology bundles $\mathcal{E}$ of monads (6]}

In this section we explore in detail the geometry of the reflexive sheaves $\mathcal{F}$ described in Lemma 23. The main result of this study will be the upper estimates for the dimensions of the moduli space of sheaves $\mathcal{F}$ and sheaves $\mathcal{L}$ obtained from $\mathcal{F}$ by the elementary transformation (170). These estimates are obtained in Propositions 26 and 27 below. This will eventually lead to the proof of Theorem 21

Denote

$$
\begin{gathered}
\mathcal{R}_{k}^{u}:=\left\{[\mathcal{F}] \in \mathcal{R}_{k} \mid \mathcal{F} \text { is unstable }\right\}, \quad \mathcal{R}_{k}^{s}:=\left\{[\mathcal{F}] \in \mathcal{R}_{k} \mid \mathcal{F} \text { is stable }\right\}, \\
\mathcal{H}_{k}^{u}:=\left\{[\mathcal{E}] \in \mathcal{H}_{k} \mid \mathcal{E} \text { is obtained from } \mathcal{F} \text { in }(179), \text { where }[\mathcal{F}] \in \mathcal{R}_{k}^{u}\right\}, \\
\mathcal{H}_{k}^{s}:=\left\{[\mathcal{E}] \in \mathcal{H}_{k} \mid \mathcal{E} \text { is obtained from } \mathcal{F} \text { in }\left(\underline{179)} \text {, where }[\mathcal{F}] \in \mathcal{R}_{k}^{s}\right\},\right.
\end{gathered}
$$

where $0 \leq k \leq 6$. Thus, $\mathcal{R}_{k}=\mathcal{R}_{k}^{u} \sqcup \mathcal{R}_{k}^{s}$ and (144) and (178) yield:

$$
\mathcal{H} \backslash(\mathcal{H} \cap \mathcal{G}(2,1)) \subset \bigsqcup_{0 \leq k \leq 6}\left(\mathcal{H}_{k}^{u} \sqcup \mathcal{H}_{k}^{s}\right) .
$$

The estimate for the dimension of $\mathcal{H} \backslash(\mathcal{H} \cap \mathcal{G}(2,1))$ will eventually follow from the computations of dimensions of $\mathcal{H}_{k}^{u}$ and $\mathcal{H}_{k}^{s}$ which we will give below. For this, we start with an explicit description of the spaces $\mathcal{R}_{k}^{u}$ and $\mathcal{R}_{k}^{s}$.

Proposition 26. (i) $\mathcal{R}_{k}^{u} \neq \emptyset$ only for $0 \leq k \leq 3$, and any sheaf $\mathcal{F}$ from $\mathcal{R}_{k}^{u}$ fits in an exact triple

$$
0 \rightarrow \mathcal{O}_{\mathbb{P}^{3}} \stackrel{s}{\rightarrow} \mathcal{F} \stackrel{u}{\rightarrow} \mathcal{I}_{C, \mathbb{P}^{3}} \rightarrow 0,
$$

where $C=\operatorname{Sing}\left(\mathcal{F} / \mathcal{O}_{\mathbb{P}^{3}}\right)$ is a l.c.i. curve of degree 2 in $\mathbb{P}^{3}, \chi\left(\mathcal{O}_{C}\right)=4-\frac{1}{2} c_{3}(\mathcal{F})=4-k$.

(ii) If $C$ is reduced, then either $c_{3}(\mathcal{F})=4$ and $C$ is a disjoint union $l_{1} \sqcup l_{2}$ of two projective lines in $\mathbb{P}^{3}$, or $c_{3}(\mathcal{F})=6$, then $C$ is a plane conic in $\mathbb{P}^{3}$.

(iii) If $C$ is nonreduced then $C$ is the scheme structure of multiplicity two on a projective line $l$ in $\mathbb{P}^{3}$ defined by an exact sequence

$$
0 \rightarrow \mathcal{I}_{C, \mathbb{P}^{3}} \rightarrow \mathcal{I}_{l, \mathbb{P}^{3}} \rightarrow \mathcal{O}_{l}(m) \rightarrow 0, \quad-1 \leq m=2-k \leq 2 .
$$

(iv) The moduli spaces $\mathcal{R}_{k}^{u}$ are varieties of dimensions

$$
\operatorname{dim} \mathcal{R}_{0}^{u}=\operatorname{dim} \mathcal{R}_{3}^{u}=14, \quad \operatorname{dim} \mathcal{R}_{1}^{u}=\operatorname{dim} \mathcal{R}_{2}^{u}=13,
$$

and they are fine.

Proof. (i)-(iii). By Lemma223, we have $c_{1}(\mathcal{F})=0, c_{2}(\mathcal{F})=2$. Since $\mathcal{F}$ is unstable, it follows from [20, Lemma 3.1] that $H^{0}(\mathcal{F}) \neq 0$. Besides, from (167) and the triple (169) twisted by $\mathcal{O}_{\mathbb{P}^{3}}(-1)$ we obtain $H^{0}(\mathcal{F}(-1))=0$. Take a section $0 \neq s \in H^{0}(\mathcal{F})$ and define a subscheme $C$ in $\mathbb{P}^{3}$ by the ideal sheaf $\mathcal{I}_{C, \mathbb{P}^{3}}=\operatorname{im}\left(u: \mathcal{F} \stackrel{\text { can }}{\simeq} \mathcal{F}^{\vee} \stackrel{s^{\vee}}{\longrightarrow} \mathcal{O}_{\mathbb{P}^{3}}\right)$. (The canonical isomorphism can : $\mathcal{F} \stackrel{\simeq}{\rightarrow} \mathcal{F}^{\vee}$ follows since $c_{1}(\mathcal{F})=0$.) From the equality $H^{0}(\mathcal{F}(-1))=0$, by 20 , Thm 4.1] we obtain that:

(a) $C$ is a Cohen-Macaulay curve in $\mathbb{P}^{3}$ satisfying the triple (182), so that $\operatorname{deg} C=c_{2}(\mathcal{F})=2$, and 
(b) the triple (182) is exact, and the equality $\chi\left(\mathcal{O}_{C}\right)=4-k$ follows from this triple and [20, Thm. 2.3]; moreover, (182) defines an extension

$$
\xi \in \operatorname{Ext}^{1}\left(\mathcal{I}_{C, \mathbb{P}^{3}}, \mathcal{O}_{\mathbb{P}^{3}}\right) \simeq H^{0}\left(\mathcal{E} x t^{1}\left(\mathcal{I}_{C, \mathbb{P}^{3}}, \mathcal{O}_{\mathbb{P}^{3}}\right)\right) \simeq H^{0}\left(\mathcal{E} x t^{2}\left(\mathcal{O}_{C}, \mathcal{O}_{\mathbb{P}^{3}}\right)\right) .
$$

(Here we use standard isomorphisms relatig global Ext-groups and $\mathcal{E} x t$-sheaves - see [20, Sec. 4].) If $C$ is a reduced curve then, since $\operatorname{deg} C=2, C$ is either a disjoint union $l_{1} \sqcup l_{2}$ of lines, or a conic. If $C$ is nonreduced, then $C$ is the scheme structure of multiplicity two on a projective line $l$ (in the sense of [12, Definition on p. 58]). Moreover, since $C$ is Cohen-Macaulay, the sheaf $\mathcal{I}_{l, \mathbb{P}^{3}} / \mathcal{I}_{C, \mathbb{P}^{3}}$ has no 0-dimensional torsion. Hence, by [12, Claim on p. 59], the exact triple (183) follows and, moreover, $C$ is a locally complete intersection. The triples (183) and (182) yield the equality $m=2-\frac{1}{2} c_{3}(\mathcal{F})=2$-k. Furthermore, (183) and the isomorphism

$$
\left.\mathcal{I}_{l, \mathbb{P}^{3}}\right|_{l} \simeq N_{l / \mathbb{P}^{3}}^{\vee} \simeq \mathcal{O}_{l}(-1)^{\oplus 2}
$$

imply $m \geq-1$. Besides, $2-m=k=\frac{1}{2} c_{3}(\mathcal{F}) \geq 0$, as $\mathcal{F}$ is reflexive. Thus, $-1 \leq m \leq 2$ and therefore $0 \leq k \leq 3$. (iv) Consider the varieties $\mathcal{C}_{k}=\left\{C \mid C\right.$ is a l.c.i. curve of degree 2 in $\left.\mathbb{P}^{3}, \chi\left(\mathcal{O}_{C}\right)=4-k\right\}, 0 \leq k \leq 3$. From (i)-(iii) and [12, Remark 1.3] it follows that $\mathcal{C}_{k}$ are rational varieties of dimensions

$$
\operatorname{dim} \mathcal{C}_{0}=11, \quad \operatorname{dim} \mathcal{C}_{1}=9, \quad \operatorname{dim} \mathcal{C}_{2}=\operatorname{dim} \mathcal{C}_{3}=8
$$

Note that (183) yields an exact triple $0 \rightarrow \mathcal{O}_{l}(2-k) \rightarrow \mathcal{O}_{C} \rightarrow \mathcal{O}_{l} \rightarrow 0, k=\frac{1}{2} c_{3}(\mathcal{F})$. Applying to it the functor $\mathcal{E} x t^{2}\left(\mathcal{O}_{l}, \mathcal{O}_{\mathbb{P}^{3}}\right)$ and using the relations $\mathcal{E} x t^{2}\left(\mathcal{O}_{l}, \mathcal{O}_{\mathbb{P}^{3}}\right) \simeq \operatorname{det}\left(N_{l / \mathbb{P}^{3}}\right) \simeq \mathcal{O}_{l}(2), \mathcal{E}_{x} t^{i}\left(\mathcal{O}_{l}, \mathcal{O}_{\mathbb{P}^{3}}\right)=0, i=1,3$, (see 34, pp. 49-50]) we obtain an exact triple $0 \rightarrow \mathcal{O}_{l}(2) \rightarrow \mathcal{E} x t^{2}\left(\mathcal{O}_{C}, \mathcal{O}_{\mathbb{P}^{3}}\right) \stackrel{\epsilon}{\rightarrow} \mathcal{O}_{l}(k) \rightarrow 0$ which, together with (185), yields

$$
\begin{aligned}
& \operatorname{dim} \operatorname{Hom}\left(\mathcal{I}_{C, \mathbb{P}^{3}}, \mathcal{O}_{\mathbb{P}^{3}}\right)=1, \operatorname{Ext}^{\geq 2}\left(\mathcal{I}_{C, \mathbb{P}^{3}}, \mathcal{O}_{\mathbb{P}^{3}}\right)=0, \\
& \operatorname{dim} \operatorname{Ext}^{1}\left(\mathcal{I}_{C, \mathbb{P}^{3}}, \mathcal{O}_{\mathbb{P}^{3}}\right)=h^{0}\left(\mathcal{E} x t^{2}\left(\mathcal{O}_{C}, \mathcal{O}_{\mathbb{P}^{3}}\right)=k+4,\right.
\end{aligned}
$$

Now, by (i)-(iii), for $0 \leq k \leq 3$, the spaces $\mathcal{R}_{k}^{u}$ are described as: $\mathcal{R}_{k}^{u}=\left\{([\mathcal{F}],\langle\xi\rangle) \mid[\mathcal{F}] \in \mathcal{R}_{k}, \mathcal{F}\right.$ fits in (182), $\langle\xi\rangle \in$ $\left.\mathbb{P}\left(\operatorname{Ext}^{1}\left(\mathcal{I}_{C, \mathbb{P}^{3}}, \mathcal{O}_{\mathbb{P}^{3}}\right)\right)\right\}=\left\{(C,\langle\xi\rangle) \mid C \in \mathcal{C}_{k},\langle\xi\rangle \in \mathbb{P}\left(\operatorname{Ext}^{1}\left(\mathcal{I}_{C, \mathbb{P}^{3}}, \mathcal{O}_{\mathbb{P}^{3}}\right)\right)\right\}$. This, together with (188), shows that $\mathcal{R}_{k}$ is a projective fibration with fibre $\mathbb{P}^{k+3}$ over $\mathcal{C}_{k}$, and (187) yields (184). Note that there exist universal flat families $\Gamma \subset \mathcal{C}_{k}$ of curves $C$, and in view of (188) and [31, Thm. 1.4] the sheaves $\mathcal{E}_{x} t_{p_{\mathcal{C}_{k}}}^{i}\left(\mathcal{I}_{\Gamma, \mathcal{C}_{k}}, \mathcal{O}_{\mathcal{C}_{k}}\right)$ commute with the base change. Hence, by [31, Prop. 4.2] there exist universal sheaves $\mathcal{F}$ on $\mathcal{R}_{k}^{u}$, i. e., $\mathcal{R}_{k}^{u}$ are fine moduli spaces.

Proposition 27. Suppose that $[\mathcal{F}] \in \mathcal{R}_{k}^{s}$. Then the following statements hold.

(i) $\mathcal{R}_{k}^{s} \neq \emptyset$ only for $0 \leq k \leq 2$.

(ii) $\operatorname{dim} \mathcal{R}_{k}^{s}=13, k=0,1,2$.

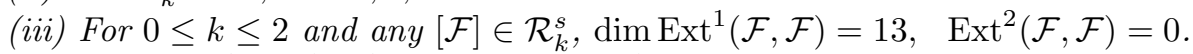

(iv) For any $\mathbb{P}^{2} \subset \mathbb{P}^{3}, h^{0}\left(\mathcal{F}_{\mathbb{P}^{2}}(2)\right)=10, h^{1}\left(\mathcal{F}_{\mathbb{P}^{2}}(2)\right)=0$.

Proof. Statements (i)-(iii) are proved in [12, Sec. 2]. The equalities in statement (iv) follow from the exact triple $0 \rightarrow \mathcal{F}(1) \rightarrow \mathcal{F}(2) \rightarrow \mathcal{F}_{\mathbb{P}^{2}}(2) \rightarrow 0$ and [12, Tables 2.8.1 and 2.12.2] for $k=2,4$ and, respectively, from [19, §9] for $k=0$.

We next proceed to a detailed description of the relation between the spaces $\mathcal{H}_{k}^{u}$ and $\mathcal{R}_{k}^{u}$ for $0 \leq k \leq 3$ and the spaces $\mathcal{H}_{k}^{s}$ and $\mathcal{R}_{k}^{s}$ for $0 \leq k \leq 2$ given by steps 1 and 2 of formula (180). Denote

$$
\begin{aligned}
{ }^{2} S_{\mathcal{F}}^{u} & :=\left\{\mathbb{P}^{2} \in \check{\mathbb{P}}^{3} \mid \operatorname{dim}\left(C \cap \mathbb{P}^{2}\right)=1, C \subset \mathbb{P}^{2}, \mathcal{O}_{C}=\mathcal{O}_{\mathbb{P}^{3}} /\left(\mathcal{F} / \mathcal{O}_{\mathbb{P}^{3}}\right)\right\}, \quad[\mathcal{F}] \in \mathcal{R}_{3}^{u}, \\
{ }^{2} S_{\mathcal{F}}^{u} & :=\emptyset, \quad[\mathcal{F}] \in \mathcal{R}_{k}^{u}, \quad k \leq 2, \\
{ }^{1} S_{\mathcal{F}}^{u} & :=\left\{\mathbb{P}^{2} \in \check{\mathbb{P}}^{3} \mid \operatorname{dim}\left(C \cap \mathbb{P}^{2}\right)=1, \mathcal{O}_{C}=\mathcal{O}_{\mathbb{P}^{3}} /\left(\mathcal{F} / \mathcal{O}_{\mathbb{P}^{3}}\right), C \not \subset \mathbb{P}^{2}\right\}, \quad[\mathcal{F}] \in \mathcal{R}_{k}^{u}, \\
{ }^{2} S_{\mathcal{F}}^{s} & ={ }^{1} S_{\mathcal{F}}^{s}:=\emptyset, \quad \text { if }[\mathcal{F}] \in \mathcal{R}_{k}^{s}, \\
{ }^{0} S_{\mathcal{F}}^{u} & :=\left\{\mathbb{P}^{2} \in \check{\mathbb{P}}^{3} \backslash\left({ }^{1} S_{\mathcal{F}}^{u} \cup{ }^{2} S_{\mathcal{F}}^{u}\right) \mid \operatorname{Sing} \mathcal{F} \cap \mathbb{P}^{2} \neq \emptyset\right\}, \quad[\mathcal{F}] \in \mathcal{R}_{k}^{u}, \\
{ }^{0} S_{\mathcal{F}}^{s} & :=\left\{\mathbb{P}^{2} \in \check{\mathbb{P}}^{3} \backslash\left({ }^{1} S_{\mathcal{F}}^{s} \cup{ }^{2} S_{\mathcal{F}}^{s}\right) \mid \operatorname{Sing} \mathcal{F} \cap \mathbb{P}^{2} \neq \emptyset\right\}, \quad[\mathcal{F}] \in \mathcal{R}_{k}^{s}, \\
{ }^{-1} S_{\mathcal{F}}^{u}:=\check{\mathbb{P}}^{3} \backslash\left({ }^{0} S_{\mathcal{F}}^{u} \cup{ }^{1} S_{\mathcal{F}}^{u} \cup{ }^{2} S_{\mathcal{F}}^{u}\right), & \\
{ }^{-1} S_{\mathcal{F}}^{s} & :=\check{\mathbb{P}}^{3} \backslash\left({ }^{0} S_{\mathcal{F}}^{s} \cup{ }^{1} S_{\mathcal{F}}^{s} \cup{ }^{2} S_{\mathcal{F}}^{s}\right), \\
\mathcal{D}_{k}^{u} & :=\mathcal{R}_{k}^{u} \times \check{\mathbb{P}}^{3}, \quad{ }^{i} \mathcal{D}_{k}^{u}:=\left\{\left([\mathcal{F}], \mathbb{P}^{2}\right) \in \mathcal{D}_{k}^{u} \mid \mathbb{P}^{2} \in{ }^{i} S_{\mathcal{F}}^{u}\right\}, \quad-1 \leq i \leq 2, \\
\mathcal{D}_{k}^{s} & :=\mathcal{R}_{k}^{s} \times \check{\mathbb{P}}^{3}, \quad{ }^{i} \mathcal{D}_{k}^{s}:=\left\{\left([\mathcal{F}], \mathbb{P}^{2}\right) \in \mathcal{D}_{k}^{s} \mid \mathbb{P}^{2} \in{ }^{i} S_{\mathcal{F}}^{s}\right\}, \quad-1 \leq i \leq 2, \\
\mathcal{D}_{k}: & :=\mathcal{D}_{k}^{u} \sqcup \mathcal{D}_{k}^{s}={ }_{-1 \leq i \leq 2} \sqcup \mathcal{D}_{k}, \quad \text { where }{ }^{i} \mathcal{D}_{k}:={ }^{i} \mathcal{D}_{k}^{u} \sqcup{ }^{i} \mathcal{D}_{k}^{s}, \quad-1 \leq i \leq 2 .
\end{aligned}
$$


Clearly, ${ }^{i} \mathcal{D}_{k}^{u}$ (respectively, ${ }^{i} \mathcal{D}_{k}^{s}$ ) are locally closed in $\mathcal{D}_{k}^{u}$ (respectively, $\mathcal{D}_{k}^{s}$ ) and

$$
\mathcal{D}_{k}^{u}=\bigsqcup_{-1 \leq i \leq 2}{ }^{i} \mathcal{D}_{k}^{u}, \quad \mathcal{D}_{k}^{s}=\bigsqcup_{-1 \leq i \leq 2}{ }^{i} \mathcal{D}_{k}^{s},
$$

Next, denote

$$
\operatorname{dim}^{i} \mathcal{D}_{k}^{u} \leq \operatorname{dim} \mathcal{R}_{k}^{u}+2-i, \quad \operatorname{dim}^{i} \mathcal{D}_{k}^{s} \leq \operatorname{dim} \mathcal{R}_{k}^{s}+2-i, \quad-1 \leq i \leq 2 .
$$

$$
\begin{aligned}
& \Pi\left(\mathcal{F}, \mathbb{P}^{2}\right):=\left\{\langle\rho\rangle \in \mathbb{P}\left(\operatorname{Hom}\left(\mathcal{F}, \mathcal{O}_{\mathbb{P}^{2}}(2)\right)\right) \mid \operatorname{im}\left(\rho: \mathcal{F} \rightarrow \mathcal{O}_{\mathbb{P}^{2}}(2)\right)=\mathcal{I}_{Z, \mathbb{P}^{2}}(2)\right. \\
& \text { for a subscheme } \left.Z \subset \mathbb{P}^{2}, \operatorname{dim} Z \leq 0, \ell(Z)=6-k\right\}, \quad\left([\mathcal{F}], \mathbb{P}^{2}\right) \in \mathcal{D}_{k},
\end{aligned}
$$

$$
K_{\left([\mathcal{F}], \mathbb{P}^{2}\right)}:=\left\{\langle\rho\rangle \in \Pi\left(\mathcal{F}, \mathbb{P}^{2}\right) \mid\left[\mathcal{L}_{\rho}:=(\operatorname{ker} \rho)(1)\right] \in \mathcal{R}(1,4,6) \text { is stable }\right\},
$$$$
\left([\mathcal{F}], \mathbb{P}^{2}\right) \in \mathcal{D}_{k}, \mathcal{Q}_{k}:=\left\{\left([\mathcal{F}], \mathbb{P}^{2},\langle\rho\rangle\right) \mid\left([\mathcal{F}], \mathbb{P}^{2}\right) \in \mathcal{D}_{k},\langle\rho\rangle \in K_{\left([\mathcal{F}], \mathbb{P}^{2}\right)}\right\},
$$$$
\mathcal{Q}_{k} \stackrel{p_{1 k}}{\longrightarrow} \mathcal{D}_{k} \text { is the forgetful map, } p_{1 k}^{-1}\left([\mathcal{F}], \mathbb{P}^{2}\right)=K_{\left([\mathcal{F}], \mathbb{P}^{2}\right)},
$$

$$
\begin{aligned}
& \Sigma_{\left([\mathcal{F}], \mathbb{P}^{2},\langle\rho\rangle\right)}:=\left\{\langle\gamma\rangle \in \mathbb{P}\left(\operatorname{Hom}\left(\mathcal{L}_{\rho}, \mathcal{O}_{\mathbb{P}^{2}}(2)\right)\right) \mid \gamma: \mathcal{L}_{\rho} \rightarrow \mathcal{O}_{\mathbb{P}^{2}}(2)\right. \text { is an } \\
& \text { epimorphism and ker } \gamma \text { is locally free }\}, \quad\left([\mathcal{F}], \mathbb{P}^{2},\langle\rho\rangle\right) \in \mathcal{Q}_{k},
\end{aligned}
$$$$
T_{k}:=\left\{\left([\mathcal{F}], \mathbb{P}^{2},\langle\rho\rangle,\langle\gamma\rangle\right) \mid\left([\mathcal{F}], \mathbb{P}^{2},\langle\rho\rangle\right) \in \mathcal{Q}_{k},\langle\gamma\rangle \in \Sigma_{\left([\mathcal{F}], \mathbb{P}^{2},\langle\rho\rangle\right)}\right\},
$$$$
T_{k} \stackrel{p_{2 k}}{\longrightarrow} \mathcal{Q}_{k} \text { is the forgetful map, } p_{2 k}^{-1}\left([\mathcal{F}], \mathbb{P}^{2},\langle\rho\rangle\right)=\Sigma_{\left([\mathcal{F}], \mathbb{P}^{2},\langle\rho\rangle\right)},
$$

$$
\begin{array}{ll}
\mathcal{Q}_{k}^{u}:=p_{1 k}^{-1}\left(\mathcal{D}_{k}^{u} \cap p_{1 k}\left(\mathcal{Q}_{k}\right)\right), & T_{k}^{u}:=p_{2 k}^{-1}\left(\mathcal{Q}_{k}^{u} \cap p_{2 k}\left(T_{k}\right)\right), \\
\mathcal{Q}_{k}^{s}:=p_{1 k}^{-1}\left(\mathcal{D}_{k}^{s} \cap p_{1 k}\left(\mathcal{Q}_{k}\right)\right), & T_{k}^{s}:=p_{2 k}^{-1}\left(\mathcal{Q}_{k}^{s} \cap p_{2 k}\left(T_{k}\right)\right) .
\end{array}
$$

(Here $0 \leq k \leq 3$ and $0 \leq k \leq 2$ in unstable case and stable case, respectively.) Since $\mathcal{D}_{k}=\mathcal{D}_{k}^{u} \sqcup \mathcal{D}_{k}^{s}$, it follows that

$$
\mathcal{Q}_{k}=\mathcal{Q}_{k}^{u} \sqcup \mathcal{Q}_{k}^{s}, \quad T_{k}=T_{k}^{u} \sqcup T_{k}^{s}, \quad 0 \leq k \leq 3 .
$$

(For consistency, in (195) and below we set $\mathcal{Q}_{3}^{s}=T_{3}^{s}=\emptyset$.) Since the stability of the sheaf $\mathcal{L}_{\rho}$ is an open property in flat families [24, Prop. 2.3.1] it follows that

$$
p_{1 k}^{-1}\left([\mathcal{F}], \mathbb{P}^{2}\right)=K_{\left([\mathcal{F}], \mathbb{P}^{2}\right)} \stackrel{\text { open }}{\longrightarrow} \Pi\left(\mathcal{F}, \mathbb{P}^{2}\right), \quad\left([\mathcal{F}], \mathbb{P}^{2}\right) \in \mathcal{D}_{k} .
$$

Take any point $\left([\mathcal{F}], \mathbb{P}^{2},\langle\rho\rangle,\langle\gamma\rangle\right)$ Since by definition $\left[\mathcal{L}_{\rho}\right] \in \mathcal{R}(1,4,6)$ is stable and $\mathcal{E}=\operatorname{ker} \gamma$ is a vector bundle, we obtain from the second triple (180) that $[\mathcal{E}] \in \mathcal{R}(0,5,0)$ is also stable, i. e. $[\mathcal{E}] \in \mathcal{B}(5)$. Thus, we obtain a natural map

$$
f_{k}: T_{k} \rightarrow \mathcal{B}(5), \quad\left([\mathcal{F}], \mathbb{P}^{2},\langle\rho\rangle,\langle\gamma\rangle\right) \mapsto[\operatorname{ker} \gamma]
$$

and by Proposition 25, $\mathcal{H}_{k}^{u} \subset f_{k}\left(T_{k}^{u}\right), \mathcal{H}_{k}^{s} \subset f_{k}\left(T_{k}^{s}\right)$. This, together with (181) and and the second formula (195) yields

$$
\mathcal{H} \backslash(\mathcal{H} \cap \mathcal{G}(2,1)) \subset \bigsqcup_{0 \leq k \leq 3} f_{k}\left(T_{k}\right) .
$$

It will follow from computations below that $T_{k}$ are disjoint unions of schemes and $f_{k}$ are morphisms for each of these schemes and all admissible values of $k$.

Lemma 28. Let $\left([\mathcal{F}], \mathbb{P}^{2}\right) \in \mathcal{D}_{k}$ and $\Pi\left(\mathcal{F}, \mathbb{P}^{2}\right) \neq \emptyset$. Then:

(i) there is an open embedding $j: \Pi\left(\mathcal{F}, \mathbb{P}^{2}\right) \stackrel{\text { open }}{\longrightarrow} \mathbb{P}\left(H^{0}\left(\left(\mathcal{F}_{\mathbb{P}^{2}}\right)^{\vee \vee}(2)\right)\right)$ and for any $\langle\rho\rangle \in \Pi\left(\mathcal{F}, \mathbb{P}^{2}\right)$ there exists a subscheme $W(\rho)$ of $\mathbb{P}^{2}, \operatorname{dim} W(\rho) \leq 0$, and an exact triple

$$
0 \rightarrow \mathcal{F}_{\mathbb{P}^{2}}(2) \stackrel{\text { can }}{\longrightarrow}\left(\mathcal{F}_{\mathbb{P}^{2}}\right)^{\vee \vee}(2) \rightarrow \mathcal{O}_{W(\rho)} \rightarrow 0, \quad \ell_{W(\rho)}=k ;
$$

(ii) if $\Sigma_{\left([\mathcal{F}], \mathbb{P}^{2},\langle\rho\rangle\right)} \neq \emptyset$ for $\left([\mathcal{F}], \mathbb{P}^{2},\langle\rho\rangle\right) \in \mathcal{Q}_{k}$, then there is an open embedding $\Sigma_{\left([\mathcal{F}], \mathbb{P}^{2},\langle\rho\rangle\right)} \stackrel{\text { open }}{\longrightarrow} \mathbb{P}\left(\operatorname{Hom}\left(\mathcal{L}, \mathcal{O}_{\mathbb{P}^{2}}(2)\right)\right) \simeq$ $\mathbb{P}^{10}$;

(iii) if $\left([\mathcal{F}], \mathbb{P}^{2}\right) \in{ }^{-1} \mathcal{D}_{k}$, then $k=0, h^{0}\left(\mathcal{F}_{\mathbb{P}^{2}}^{\vee \vee}(2)\right)=h^{0}\left(\mathcal{F}_{\mathbb{P}^{2}}(2)\right)=10$;

(iv) if ${ }^{0} \mathcal{D}_{k} \neq \emptyset$ and $\left([\mathcal{F}], \mathbb{P}^{2}\right) \in{ }^{0} \mathcal{D}_{k}$, then $1 \leq k \leq 2, h^{0}\left(\mathcal{F}_{\mathbb{P}^{2}}(2)\right)=10$ and

$$
h^{0}\left(\left(\mathcal{F}_{\mathbb{P}^{2}}\right)^{\vee \vee}(2)\right)=10+k
$$


(v) if ${ }^{1} \mathcal{D}_{k} \cup{ }^{2} \mathcal{D}_{k} \neq \emptyset$ and $\left([\mathcal{F}], \mathbb{P}^{2}\right) \in{ }^{1} \mathcal{D}_{k} \cup{ }^{2} \mathcal{D}_{k}$, then the equalities (200) hold for $k=1,2,3$ and

$$
h^{0}\left(\left(\mathcal{F}_{\mathbb{P}^{2}}\right)^{\vee \vee}(2)\right)=h^{0}\left(\left(\mathcal{F}_{\mathbb{P}^{2}}\right)(2)\right)=11, \quad \text { if } \quad k=0 .
$$

Proof. (i) Take any $\langle\rho\rangle \in \Pi\left(\mathcal{F}, \mathbb{P}^{2}\right)$. By the definition of $\Pi\left(\mathcal{F}, \mathbb{P}^{2}\right)$ we may consider $\rho$ as a composition $\rho: \mathcal{F} \stackrel{\otimes \mathcal{O}_{\mathbb{P}}^{2}}{\longrightarrow}$

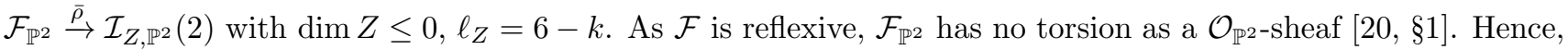
$\operatorname{ker} \bar{\rho}$ is a rank 1 torsion free $\mathcal{O}_{\mathbb{P}^{2}}$-sheaf. Since $c_{1}\left(\mathcal{F}_{\mathbb{P}^{2}}\right)=0, c_{2}\left(\mathcal{F}_{\mathbb{P}^{2}}\right)=2$, $\operatorname{ker} \bar{\rho} \simeq \mathcal{I}_{W, \mathbb{P}^{2}}(-2)$, where $\operatorname{dim} W \leq 0$, and there is an exact triple

$$
0 \rightarrow \mathcal{I}_{W, \mathbb{P}^{2}} \stackrel{\theta_{\rho}}{\rightarrow} \mathcal{F}_{\mathbb{P}^{2}}(2) \stackrel{\bar{\rho}}{\rightarrow} \mathcal{I}_{Z, \mathbb{P}^{2}}(4) \rightarrow 0 .
$$

The monomorphism $\theta=\theta_{\rho}$ in this triple extends to a commutative square

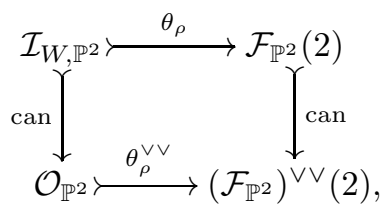

and we obtain a morphism $j: \Pi\left(\mathcal{F}, \mathbb{P}^{2}\right) \rightarrow \mathbb{P}\left(H^{0}\left(\left(\mathcal{F}_{\mathbb{P}^{2}}\right)^{\vee}(2)\right)\right),\langle\rho\rangle \mapsto \theta_{\rho}^{\vee \vee}$. To construct the inverse to $j$ morphism $\psi:(\operatorname{imj}) \rightarrow \Pi\left(\mathcal{F}, \mathbb{P}^{2}\right)$, take any $\left(\tilde{\theta}: \mathcal{O}_{\mathbb{P}^{2}} \rightarrow\left(\mathcal{F}_{\mathbb{P}^{2}}\right)^{\vee \vee}(2)\right) \in \operatorname{im} j$. The morphism $\theta: \mathcal{I}_{W, \mathbb{P}^{2}} \rightarrow \mathcal{F}_{\mathbb{P}^{2}}(2)$ such that $\tilde{\theta}=\theta^{\vee \vee}$ is recovered from $\tilde{\theta}$ as $\left.\tilde{\theta}\right|_{\mathcal{I}_{W, \mathbb{P}^{2}}}$, where $\mathcal{I}_{W, \mathbb{P}^{2}}=\operatorname{can}^{-1}\left(\tilde{\theta}\left(\mathcal{O}_{\mathbb{P}^{2}}\right) \cap \operatorname{can}\left(\mathcal{F}_{\mathbb{P}^{2}}(2)\right)\right)$. Then $\bar{\theta}$ defines via $\theta$ a morphism $\bar{\rho}$ as the quotient morphism $\mathcal{F}_{\mathbb{P}^{2}}(2) \rightarrow \operatorname{coker} \theta \simeq \mathcal{I}_{Z, \mathbb{P}^{2}}(4)$, and we set $\psi(\langle\bar{\theta}\rangle):=\left\langle\bar{\rho} \circ\left(-\otimes \mathcal{O}_{\mathbb{P}^{2}}\right)\right\rangle$. The openness of $j$ follows from the openness of the condition $\rho: \mathcal{F} \rightarrow \mathcal{O}_{\mathbb{P}^{2}}(2)$ to be surjective.

Next, remark that, in (203), the $\mathcal{O}_{\mathbb{P}^{2}}$-sheaf $\operatorname{coker} \theta \simeq \mathcal{I}_{Z, \mathbb{P}^{2}}(4)$ has no torsion, hence there is no nonzero morphism $\mathcal{O}_{W}=\mathcal{O}_{\mathbb{P}^{2}} / \mathcal{I}_{W, \mathbb{P}^{2}} \rightarrow \operatorname{coker} \theta$, since $\operatorname{dim} W \leq 0$. Thus, (203) and the Snake Lemma yield an exact triple (199) with $W(\rho)=W$.

(ii) The injection $\Sigma_{\left([\mathcal{F}], \mathbb{P}^{2},\langle\rho\rangle\right)} \hookrightarrow \mathbb{P}\left(\operatorname{Hom}\left(\mathcal{L}_{\rho}, \mathcal{O}_{\mathbb{P}^{2}}(2)\right)\right) \simeq \mathbb{P}^{10}$ is an open embedding since the condition that $\gamma: \mathcal{L}:=\mathcal{L}_{\rho} \rightarrow \mathcal{O}_{\mathbb{P}^{2}}(2)$ is an epimorphism and ker $\gamma$ is locally free is open on $\langle\gamma\rangle \in \mathbb{P}\left(\operatorname{Hom}\left(\mathcal{L}, \mathcal{O}_{\mathbb{P}^{2}}(2)\right)\right)$. We thus have to show that $\operatorname{dim} \operatorname{Hom}\left(\mathcal{L}, \mathcal{O}_{\mathbb{P}^{2}}(2)\right)=11$. Consider the epimorphism $\bar{\gamma}=\left.\gamma\right|_{\mathbb{P}^{2}}: \mathcal{L}_{\mathbb{P}^{2}} \rightarrow \mathcal{O}_{\mathbb{P}^{2}}(2)$. Since by definition $[\mathcal{L}] \in \mathcal{R}(1,4,6)$, it follows that $\operatorname{ker} \bar{\gamma} \simeq \mathcal{I}_{Y, \mathbb{P}^{2}}(-1)$ for some subscheme $Y$ of $\mathbb{P}^{2}, \operatorname{dim} Y=0, \ell_{Y}=6$. This yields an exact triple $0 \rightarrow \mathcal{I}_{Y, \mathbb{P}^{2}}(-1) \rightarrow \mathcal{L}_{\mathbb{P}^{2}} \stackrel{\bar{\gamma}}{\rightarrow} \mathcal{O}_{\mathbb{P}^{2}}(2) \rightarrow 0$. Applying to it the functor $\mathcal{E} t_{\mathcal{O}_{\mathbb{P}^{2}}}\left(-, \mathcal{O}_{\mathbb{P}^{2}}(2)\right)$ we obtain an exact triple $0 \rightarrow \mathcal{O}_{\mathbb{P}^{2}} \rightarrow \mathcal{H o m}\left(\mathcal{L}_{\mathbb{P}^{2}}, \mathcal{O}_{\mathbb{P}^{2}}(2)\right) \rightarrow \mathcal{O}_{\mathbb{P}^{2}}(3) \rightarrow 0$ which implies $\operatorname{dim} \operatorname{Hom}\left(\mathcal{L}, \mathcal{O}_{\mathbb{P}^{2}}(2)\right)=$ $\operatorname{dim} \operatorname{Hom}\left(\mathcal{L}_{\mathbb{P}^{2}}, \mathcal{O}_{\mathbb{P}^{2}}(2)\right)=h^{0}\left(\mathcal{H} o m\left(\mathcal{L}_{\mathbb{P}^{2}}, \mathcal{O}_{\mathbb{P}^{2}}(2)\right)\right)=11$.

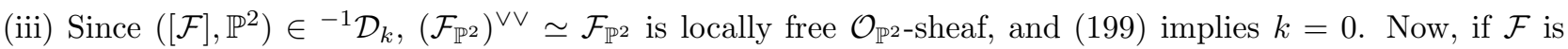
unstable, then applying to (182) the functor $-\otimes \mathcal{O}_{\mathbb{P}^{2}}(2)$ we have an exact triple

$$
0 \rightarrow \mathcal{O}_{\mathbb{P}^{2}} \rightarrow \mathcal{F}_{\mathbb{P}^{2}} \rightarrow \mathcal{I}_{Y, \mathbb{P}^{2}}(2) \rightarrow 0, \quad \operatorname{dim} Y=0, \quad \ell_{Y}=\operatorname{deg} C=2,
$$

and this triple yields the desired values of $h^{i}\left(\left(\mathcal{F}_{\mathbb{P}^{2}}\right)^{\vee \vee}(2)\right)=h^{i}\left(\mathcal{F}_{\mathbb{P}^{2}}(2)\right)$. If $\mathcal{F}$ is stable, then these values are given by Proposition 27,(iv).

(iv) Since $\left([\mathcal{F}], \mathbb{P}^{2}\right) \in{ }^{0} \mathcal{D}_{k} \cup{ }^{2} \mathcal{D}_{k} \neq \emptyset$, the morphism can in (199) is not an isomorphism, hence $k=\ell_{W(\rho)} \geq 1$. On the other hand, $k \leq 3$ by Propositions 27(i) and 26(i). As above, if $\mathcal{F}$ is unstable, the triple (204) is true, which yields the equalities $h^{0}\left(\mathcal{F}_{\mathbb{P}^{2}}(2)\right)=10, h^{1}\left(\mathcal{F}_{\mathbb{P}^{2}}(2)\right)=0$. Respectively, if $\mathcal{F}$ is stable, these equalities follow from Proposition 27(iv). Whence, by (199), we have (200).

We only have to show that, in case $\mathcal{F}$ is unstable, $k \leq 2$. By the definition of the sets ${ }^{i} \mathcal{D}_{k}^{u}, i=0,1$, the condition $\left([\mathcal{F}], \mathbb{P}^{2}\right) \in{ }^{0} \mathcal{D}_{k}^{u}$ implies that $\mathbb{P}^{2} \notin{ }^{1} S_{\mathcal{F}}^{u}$. This means that the exact triple (204) is true, with $\operatorname{dim} Y=0, \ell_{Y}=\operatorname{deg} C=2$. Dualizing this $\mathcal{O}_{\mathbb{P}^{2}}$-triple we easily obtain an inequality $h^{0}\left(\mathcal{E}_{x} t^{1}\left(\mathcal{F}_{\mathbb{P}^{2}}, \mathcal{O}_{\mathbb{P}^{2}}\right)\right) \leq$ $h^{0}\left(\mathcal{E} x t^{2}\left(\mathcal{O}_{Y}, \mathcal{O}_{\mathbb{P}^{2}}\right)\right)=\ell_{Y}=2$ and an exact tripe $0 \rightarrow \mathcal{O}_{\mathbb{P}^{2}} \rightarrow\left(\mathcal{F}_{\mathbb{P}^{2}}\right)^{\vee} \rightarrow \mathcal{I}_{Z, \mathbb{P}^{2}}(2) \rightarrow 0$ for some scheme $Z \subset \mathbb{P}^{2}$ with $\operatorname{dim} Z \leq 0, \ell_{Z}=2-h^{0}\left(\mathcal{E} x t^{1}\left(\mathcal{F}_{\mathbb{P}^{2}}, \mathcal{O}_{\mathbb{P}^{2}}\right)\right)$. This triple, together with the triple (204) and the isomorphism $\left(\mathcal{F}_{\mathbb{P}^{2}}\right)^{\vee} \simeq\left(\mathcal{F}_{\mathbb{P}^{2}}\right)^{\vee \vee}$, yields an exact triple $0 \rightarrow \mathcal{F}_{\mathbb{P}^{2}}(2) \stackrel{\text { can }}{\longrightarrow}\left(\mathcal{F}_{\mathbb{P}^{2}}\right)^{\vee \vee}(2) \rightarrow K \rightarrow 0$, where $K$ is an artinian

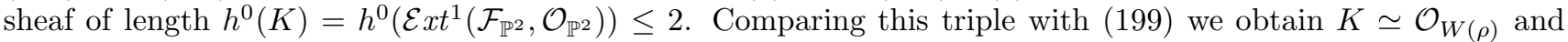
$k=\ell_{W(\rho)} \leq 2$.

(v) From the condition $\left([\mathcal{F}], \mathbb{P}^{2}\right) \in{ }^{1} \mathcal{D}_{k} \neq \emptyset$ and Proposition $\left[26\right.$ it follows that $\mathbb{P}^{2} \cap \operatorname{Sing} \mathcal{F}=l$ is a line, if $k=0,1,2$; respectively, $\mathbb{P}^{2} \cap \operatorname{Sing} \mathcal{F}=C$ is a conic, if $k=3$. Thus, applying to the triples (182) and (183) the functor $-\otimes \mathcal{O}_{\mathbb{P}^{2}}(2)$ and using the resolution $0 \rightarrow \mathcal{O}_{\mathbb{P}^{3}}(-1) \rightarrow \mathcal{O}_{\mathbb{P}^{3}} \rightarrow \mathcal{O}_{\mathbb{P}^{2}} \rightarrow 0$, we obtain the following exact 
triples, where $\operatorname{dim} W=0$ and if $k=0$ or 1 , then $W \subset l$ :

$$
\begin{aligned}
0 & \left.\rightarrow \mathcal{O}_{\mathbb{P}^{2}}(2) \rightarrow \mathcal{F}_{\mathbb{P}^{2}}(2) \rightarrow \mathcal{I}_{C, \mathbb{P}^{3}}(2)\right|_{\mathbb{P}^{2}} \rightarrow 0, \\
0 & \left.\rightarrow \mathcal{O}_{l}(3-k) \rightarrow \mathcal{I}_{C, \mathbb{P}^{3}}(2)\right|_{\mathbb{P}^{2}} \rightarrow \mathcal{I}_{W, \mathbb{P}^{2}}(1) \rightarrow 0, \quad \ell_{W}=3-k, k \leq 2, \\
0 & \left.\rightarrow \mathcal{O}_{l} \rightarrow \mathcal{I}_{C, \mathbb{P}^{3}}(2)\right|_{\mathbb{P}^{2}} \rightarrow \mathcal{O}_{\mathbb{P}^{2}}(1) \rightarrow 0, k=3, \quad C \not \subset \mathbb{P}^{2}, \quad \mathbb{P}^{2} \cap C=l, \\
0 & \left.\rightarrow \mathcal{O}_{C}(1) \rightarrow \mathcal{I}_{C, \mathbb{P}^{3}}(2)\right|_{\mathbb{P}^{2}} \rightarrow \mathcal{O}_{\mathbb{P}^{2}} \rightarrow 0, k=3, \quad C \subset \mathbb{P}^{2} .
\end{aligned}
$$

Since $\mathcal{F}$ is locally free for $k=0, h^{i}\left(\left(\mathcal{F}_{\mathbb{P}^{2}}\right)^{\vee \vee}(2)\right)=h^{i}\left(\mathcal{F}_{\mathbb{P}^{2}}(2)\right)$, from (205) we obtain (201). Respectively, for $k=1,2,3$, (205) and (199) imply (200).

For $0 \leq k \leq 3$, let $B \subset \mathbb{P}^{3} \times \check{\mathbb{P}}^{3}$ be the graph of incidence, $\mathcal{O}_{B}(2)=\left.\mathcal{O}_{\mathbb{P}^{3}}(2) \otimes \mathcal{O}_{\check{\mathbb{P}}^{3}}\right|_{B}$, and let $p r_{0}: \mathcal{D}_{k}^{u} \rightarrow \mathcal{D}_{k}^{u}$, $p r_{1}: \mathcal{D}_{k}^{u} \rightarrow \boldsymbol{\mathcal { R }}_{k}^{u}, \mathcal{D}_{k}^{u} \rightarrow \mathbb{P}^{3} \times \check{\mathbb{P}}^{3}$ be the projections. For each $m \geq 0$ consider the set

$$
Y=Y_{k, m}^{u}:=\left\{\left([\mathcal{F}], \mathbb{P}^{2}\right) \in \mathcal{D}_{k}^{u} \mid \operatorname{dim} \operatorname{Hom}\left(\mathcal{F}, \mathcal{O}_{\mathbb{P}^{2}}(2)\right)=m\right\}, \quad m \geq 0
$$

and set $\mathbf{Y}=p r_{0}^{-1}(Y), q_{i}=\left.p r_{i}\right|_{\mathbf{Y}}, i=0,1,2, L=\mathcal{E} x t_{q_{0}}\left(q_{1}^{*} \mathcal{F}, q_{2}^{*} \mathcal{O}_{B}(2)\right)$, where $\mathcal{F}$ is the universal sheaf on $\mathcal{R}_{k}^{u}$ which exists by Proposition [26] (iv), $\mathcal{Y}=\mathbf{Y} \times_{Y} \mathbf{P}\left(L^{\vee}\right)$, and let $\mathbf{P}\left(L^{\vee}\right) \stackrel{\lambda}{\leftarrow} \mathcal{Y} \stackrel{\pi}{\rightarrow} \mathbf{Y}$ and $\mathcal{Y} \stackrel{\mu}{\rightarrow} \mathbf{P}\left(L^{\vee}\right) \stackrel{\nu}{\rightarrow} Y$ be the projections. By [2, Satz 3], $Y=Y_{k, m}^{u}$ is locally closed in $\mathcal{D}_{k}^{u}$ and the sheaf $L$ is a rank $m$ locally free sheaf on $Y$ which commutes with the base change, i. e., for $y=\left([\mathcal{F}], \mathbb{P}^{2}\right) \in Y$, one has $\left.L\right|_{y}=\operatorname{Hom}\left(\mathcal{F}, \mathcal{O}_{\mathbb{P}^{2}}(2)\right)$. On $\mathcal{Y}$ there is a universal morphism $\boldsymbol{\rho}:\left(q_{1} \circ \pi\right)^{*} \mathcal{F} \rightarrow\left(q_{2} \circ \pi\right)^{*} \mathcal{O}_{B}(2) \otimes \mu^{*} \mathcal{O}_{\mathbf{P}\left(L^{\vee}\right)}(1)$. Consider the set

$$
X=X_{k, m}^{u}:=\left\{\left([\mathcal{F}], \mathbb{P}^{2}\right) \in Y_{k, m}^{u} \mid K_{\left([\mathcal{F}], \mathbb{P}^{2}\right)} \neq \emptyset\right\} .
$$

From this definition it follows that the sheaf im $\boldsymbol{\rho}$ is flat over $\mathbf{P}\left(L^{\vee}\right)$ at any point $x \in \nu^{-1}(X)$. This implies that $X$ is an open (possibly, empty) subset of $Y$, hence it is locally closed in $\mathcal{D}_{k}^{u}$. Therefore, since in view of Proposition 26] (iv) $\mathcal{D}_{k}^{u}$ are varieties, the set $\Phi_{k}^{u}=\left\{m \in \mathbb{Z}_{\geq 0} \mid X_{k, m}^{u} \neq \emptyset\right\}$ is finite. By the definitions (191), (192), (194) and (207) we have

$$
X_{k}^{u}:=\bigsqcup_{m \in \Phi_{k}^{u}} X_{k, m}^{u}=p_{1 k}\left(\mathcal{Q}_{k}^{u}\right), \quad \mathcal{Q}_{k}^{u}=p_{1 k}^{-1}\left(X_{k}^{u}\right) .
$$

Denoting ${ }^{i} X_{k}^{u}={ }^{i} \mathcal{D}_{k}^{u} \cap X_{k}^{u},{ }^{i} \mathcal{Q}_{k}^{u}=p_{1 k}^{-1}\left({ }^{i} X_{k}^{u}\right),-1 \leq i \leq 2$, we find from the first equality (189) that

$$
\mathcal{Q}_{k}^{u}=\bigsqcup_{-1 \leq i \leq 2}{ }^{i} \mathcal{Q}_{k}^{u}
$$

The inclusion (196) and Lemma 28,(i) yield that the projection $p_{1 k}:{ }^{i} \mathcal{Q}_{k}^{u} \rightarrow{ }^{i} X_{k}^{u}$ decomposes as

$$
p_{1 k}:{ }^{i} \mathcal{Q}_{k}^{u} \stackrel{\text { open }}{\longrightarrow}{ }^{i} \tilde{\mathcal{Q}}_{k}^{u} \stackrel{\tilde{p}_{1 k}}{\longrightarrow}{ }^{i} X_{k}^{u}, \quad-1 \leq i \leq 2, \quad 0 \leq k \leq 3,
$$

where ${ }^{i} \tilde{\mathcal{Q}}_{k}^{u} \stackrel{\tilde{p}_{1 k}}{\longrightarrow}{ }^{i} X_{k}^{u}$ is the projective fibration with fibre $\mathbb{P}\left(H^{0}\left(\left(\mathcal{F}_{\mathbb{P}^{2}}\right)^{\vee \vee}(2)\right)\right)$ over an arbitrary point $\left([\mathcal{F}], \mathbb{P}^{2}\right) \in$ ${ }^{i} X_{k}^{u}$. Here by (208) each ${ }^{i} X_{k}^{u}$ is a disjoint union of schemes, This shows that each ${ }^{i} \mathcal{Q}_{k}^{u}$ is a disjoint union of schemes. Since ${ }^{i} X_{k}^{u} \subset \mathcal{D}_{k}^{u}$, it follows from (190) and Lemma 28,(iii)-(v) that

$$
\operatorname{dim}^{i} \mathcal{Q}_{k}^{u} \leq \operatorname{dim}{ }^{i} \mathcal{R}_{k}^{u}+11-i+k, \quad-1 \leq i \leq 2, \quad 0 \leq k \leq 3 .
$$

Thus, in view of (184), we obtain $\operatorname{dim}^{i} \mathcal{Q}_{k}^{u} \leq 26$ for all possible $i, k$, hence (209) yields

$$
\operatorname{dim} \mathcal{Q}_{k}^{u} \leq 26, \quad 0 \leq k \leq 3 .
$$

To obtain a similar estimate for dimensions of $\mathcal{Q}_{k}^{s}$, we define similarly to (206) the locally closed subsets $Y_{k, m}^{s}:=\left\{\left([\mathcal{F}], \mathbb{P}^{2}\right) \in \mathcal{D}_{k}^{s} \mid \operatorname{dim} \operatorname{Hom}\left(\mathcal{F}, \mathcal{O}_{\mathbb{P}^{2}}(2)\right)=m\right\}, m \geq 0$, of $\mathcal{D}_{k}^{s}$. Next, note that apriori there is no universal sheaf $\mathcal{F}$ on $\mathcal{R}_{k}^{s}$. However, by Proposition 27, $\operatorname{Ext}^{2}(\mathcal{F}, \mathcal{F})=0$ for any $[\mathcal{F}] \in \mathcal{R}_{k}^{s}, 0 \leq k \leq 2$. This means that the deformation theory for $\mathcal{R}_{k}^{s}$ is unobstructed, so there exists an open cover $\mathcal{R}_{k}^{s}=\bigcup_{j \in J} \mathcal{U}_{j}$ and universal sheaves $\mathcal{F}_{j}$ on $\mathcal{U}_{j}$ (see, e. g., [9, Appendice A1-A2], [17, Ch. 6]). The existence of these local universal sheaves is enough to show that the sets $X_{k, m}^{s}$ defined similarly to (207) as $X_{k, m}^{s}:=\left\{\left([\mathcal{F}], \mathbb{P}^{2}\right) \in Y_{k, m}^{s} \mid K_{\left([\mathcal{F}], \mathbb{P}^{2}\right)} \neq \emptyset\right\}$, are locally closed subsets of $\mathcal{D}_{k}^{s}$. We then have, similarly to (208)-(209), a finite dijoint unions of schemes $X_{k}^{s}:=\bigsqcup_{m \in \Phi_{k}^{s}} X_{k, m}^{s}=p_{1 k}\left(\mathcal{Q}_{k}^{s}\right)$ and relations $\mathcal{Q}_{k}^{s}=p_{1 k}^{-1}\left(X_{k}^{s}\right)$. Denoting ${ }^{i} X_{k}^{s}={ }^{i} \mathcal{D}_{k}^{s} \cap X_{k}^{s},{ }^{i} \mathcal{Q}_{k}^{s}=p_{1 k}^{-1}\left({ }^{i} X_{k}^{u}\right),-1 \leq i \leq 2$, and mimicking the argument in (209)-(211) with $u$ substituted by $s$, we obtain that ${ }^{i} \mathcal{Q}_{k}^{s}$, respectively, $\mathcal{Q}_{k}^{s}$ are disjoint unions of schemes satisfying the inequalities $\operatorname{dim}^{i} \mathcal{Q}_{k}^{s} \leq \operatorname{dim}^{i} \mathcal{R}_{k}^{s}+11-i+k,-1 \leq i \leq 2,0 \leq k \leq 2$. These formulas and Proposition 27.(ii) imply the inequalities $\operatorname{dim} \mathcal{Q}_{k}^{s} \leq 26,0 \leq k \leq 2$, which, together with (212) and (195) yield

$$
\operatorname{dim} \mathcal{Q}_{k} \leq 26, \quad 0 \leq k \leq 3 .
$$


(Remind that, as in (195), we set $\mathcal{Q}_{3}^{s}=T_{3}^{s}=\emptyset$.) Now from Lemma 28,(ii) and the last formula in the display (193), similarly to (210), we obtain that $T_{k}$ are disjoint unions of schemes and projections $p_{2 k}: T_{k} \rightarrow \mathcal{Q}_{k}$ are morphisms which decompose as $p_{2 k}: T_{k} \stackrel{\text { open }}{\longrightarrow} \tilde{T}_{k} \stackrel{\tilde{p}_{2 k}}{\longrightarrow} \mathcal{Q}_{k}$, where $\tilde{T}_{k} \stackrel{\tilde{p}_{2 k}}{\longrightarrow}{ }^{i} \mathcal{Q}_{k}$ is the projective fibration with fibre $\mathbb{P}\left(H^{0}\left(\left(\mathcal{F}_{\mathbb{P}^{2}}\right)^{\vee \vee}(2)\right)\right) \simeq \mathbb{P}^{10}$ over an arbitrary point $\left([\mathcal{F}], \mathbb{P}^{2},\langle\rho\rangle\right) \in \mathcal{Q}_{k}, 0 \leq k \leq 3$. This together with (213) yields

$$
\operatorname{dim} T_{k} \leq 36, \quad 0 \leq k \leq 3 .
$$

Proof of Theorem 21. It is clear from the above that the maps $f_{k}: T_{k} \rightarrow \mathcal{B}(5)$ defined in (197) are morphisms. The inequality $\operatorname{dim}(\mathcal{H} \backslash(\mathcal{G}(2,1) \cap \mathcal{H})) \leq 36$ now follows from (198) and (214). However, by [20, Remark 3.4.1], any irreducible component of $\mathcal{B}(5)$ has dimension at least 37 . Hence, Theorem 21 follows.

\section{Components of $\mathcal{B}(5)$}

We finally have at hand all the results needed to complete the proof of our second main result, namely, the characterization of the irreducible components of $\mathcal{B}(5)$ given by Main Theorem 2. This entire section will be devoted to this goal.

Proof of Main Theorem Q The first ingredient of the proof is the fact, proved by Hartshorne and Rao, that every bundle in $\mathcal{B}(5)$ is cohomology of one of the monads (2) -(16), cf. [21, Table 5.3, case 5.(1)-(4)].

Recall that for each stable rank 2 bundle $E$ on $\mathbb{P}^{3}$ with vanishing first Chern class, the number $\alpha(E):=$ $h^{1}(E(-2)) \bmod 2$ is called the Atiyah-Rees $\alpha$-invariant of $E$, see [19, Definition on p. 237]. Hartshorne showed [19, Corollary 2.4] that this number is invariant on the connected components of the moduli space of stable vector bundles on $\mathbb{P}^{3}$. One can easily check that the cohomologies of monads of the form (2) and (3) have $\alpha$-invariant equal to 0 , while the cohomologies of the other three types of monads have $\alpha$-invariant equal to 1 .

Rao showed in 35 that the family of bundles obtained as cohomology of monads of the form (3) is irreducible, of dimension 36, and it lies in a unique component of $\mathcal{B}(5)$. Since instanton bundles of charge 5 , i. e. the cohomologies of monads of the form (2), yield an irreducible family of dimension 37, it follows that the set

$$
\mathcal{I}:=\{[E] \in \mathcal{B}(5) \mid \alpha(E)=0\}
$$

forms a single irreducible component of $\mathcal{B}(5)$, of dimension 37 , whose generic point corresponds to an instanton bundle. In addition, every $[E] \in \mathcal{I}$ satisfies $\mathrm{H}^{1}(\mathcal{E} n d(E))=37$; this was originaly proved by Katsylo and Ottaviani for instanton bundles [30], and by Rao for the cohomologies of monads of the form (3) [35, Section 3]. Therefore, we also conclude that $\mathcal{I}$ is nonsingular. This completes the proof of the first part of the Main Theorem.

Our next step is to analyse those bundles with Atiyah-Rees invariant equal to 1.

Hartshorne proved in [20, Theorem 9.9] that the family $\mathcal{K}$ of stable rank 2 bundles $E$ with $c_{1}(E)=0$ and $c_{2}(E)=5$ whose spectrum is $(-2,-1,0,1,2)$ is an irreducible, nonsigular family of dimension 40 , and from the definition of spectrum one has

$$
h^{1}(\mathcal{E}(-2))=3, \quad[\mathcal{E}] \in \mathcal{K} .
$$

The bundles from $\mathcal{K}$ are precisely those given as cohomologies of monads of the form (4), cf. [21, Table 5.3, case 5.(4)], which is a particular case of a class of monads studied by Ein in [15]. It is shown in [15] that the closure $\overline{\mathcal{K}}$ of $\mathcal{K}$ in $\mathcal{B}(5)$ is an irreducible component of $\mathcal{B}(5)$ of dimension 40.

We proved in Main Theorem 1, case $a=2$, that the bundles arising as cohomology of monads of the form (5) form a dense subset $\mathcal{G}(2,1)$ of a rational irreducible component of dimension 37. Consider the set $\mathcal{H}$ of bundles arising as cohomology of monads of the form (6). Since the bundles from $\mathcal{G}(2,1) \cup \mathcal{H}$ have the spectrum $(-1,0,0,0,1)$ by [21, Table 5.3 , case $5 .(2)$ ], by we have (cf. (164))

$$
h^{1}(\mathcal{E}(-2))=1, \quad[\mathcal{E}] \in \mathcal{G}(2,1) \cup \mathcal{H},
$$

so that $\alpha(\mathcal{E})=1$, and therefore, in view of (215), $\mathcal{H} \cap \mathcal{I}=\emptyset$. Since, by Theorem 21] $\mathcal{H}$ does not constitute a component in $\mathcal{B}(5)$, it then follows from the above that $\mathcal{H} \subset \overline{\mathcal{G}(2,1)} \cup \overline{\mathcal{K}}$.

Proposition 29. $\mathcal{H} \subset \overline{\mathcal{G}(2,1)}$ and $\overline{\mathcal{K}}=\mathcal{K}$.

Proof. We only have to show that $(\mathcal{G}(2,1) \cup \mathcal{H}) \cap \overline{\mathcal{K}}=\emptyset$. Suppose by contradiction that there exists a vector bundle $[\mathcal{E}] \in(\mathcal{G}(2,1) \cup \mathcal{H}) \cap \overline{\mathcal{K}}$. By (216) and the inferior semi-continuity of the dimension of the cohomology groups of coherent sheaves, one has that $h^{1}(\mathcal{E}(-2)) \geq 3$, contrary to (217).

This last proposition finally concludes the proof of Main Theorem 2

We summarize all the information in the Main Theorem 2, and the discrete invariants of stable rank 2 bundles with $c_{1}=0$ and $c_{2}=5$ in the following table. 
TABLE 1. Irreducible components of $\mathcal{B}(5)$

\begin{tabular}{|c|c|c|c|c|}
\hline Component & Dimension & Monads & Spectra & $\alpha$-invariant \\
\hline \multirow{2}{*}{ Instanton } & 37 & $(2)$ & $(0,0,0,0,0)$ & \multirow{2}{*}{0} \\
\cline { 3 - 4 } & 40 & $(\underline{31})$ & $(-1,-1,0,1,1)$ & \\
\hline Ein & 40 & $(-2,-1,0,1,2)$ & 1 \\
\hline $\begin{array}{c}\text { Modified } \\
\text { Instanton }\end{array}$ & 37 & $(\underline{51})$ & $(-1,0,0,0,1)$ & 1 \\
\cline { 3 - 4 } & & $(6)$ & & \\
\hline
\end{tabular}

Remark. Inspired by the techniques introduced in the present paper, the authors of [38] construct another infinite series of irreducible components of $\mathcal{B}(0, n)$ whose special point corresponds to a bundle obtained as the cohomology of a monad similar to the one in display (24), just substituting a direct sum of two rank 2 instantons bundles for the rank 4 instanton bundle of charge 1 in middle term.

\section{REFERENCES}

[1] C. Banica, N. Manolache. Rank 2 stable vetor bundles on $\mathbb{P}^{3}$, with Chern Classes $c_{1}=-1, c_{2}=-4$. Math. Z. 190 (1985), 315-339.

[2] C. Banica, M. Putinar, G. Schumacher. Variation der globalen Ext in Deformationen kompakter komplexer Räume. Math. Ann. 250 (1980), no. 2, 135-155.

[3] W. Barth. Some properties of stable rank-2 vector bundles on $\mathbb{P}^{n}$. Math. Ann. 226 (1977), 125-150.

[4] W. Barth. Stable vector bundles on $\mathbb{P}^{3}$, some experimental data. Asterisque 71 (1980), 205-218.

[5] W. Barth, G. Elencwaig. Concernant la Cohomologie des fibrés Algébriques Stables sur $\mathbf{P}_{n}(\mathbb{C})$. In: Variétés Analytiques Complexes (Nice 1977). Lecture Notes in Math. 683, 1-24. Springer, Berlin, 1978.

[6] W. Barth and K. Hulek. Monads and moduli of vector bundles. Manuscr. Math. 25 (1978), 323-347.

[7] W. Barth, K. Hulek, C. Peters, A. Van de Ven. Compact complex surfaces. Second Enlarged Edition, Springer, 2004.

[8] G. Bohnhorst, H. Spindler. The stability of certain vector bundles on $\mathbb{P}^{n}$. In: Complex algebraic varieties (Bayreuth, 1990), 39-50, Lecture Notes in Math., 1507, Springer, Berlin, 1992.

[9] J. Brun, A. Hirschowitz. Variété des droites sauteuses du fibré instanton général, With an appendix by J. Bingener, Compositio Math. 53 (1984), 325-336.

[10] U. Bruzzo, D. Markushevich, A. S. Tikhomirov. Moduli of symplectic instanton vector bundles of higher rank on projective space $\mathbb{P}^{3}$. Cent. Eur. J. Math. 10 (2012), 1232-1245.

[11] M.-C. Chang. Stable rank 2 bundles on $\mathbb{P}^{3}$ with $c_{1}=0, c_{2}=4$ and $\alpha=1$. Math. Z. 184 (1983), 407-415.

[12] M.-C. Chang. Stable rank 2 reflexive sheaves on $\mathbb{P}^{3}$ with small $c_{2}$ and applications. Trans. Amer. Math. Soc. 284 (1984), no. 1, $57-89$.

[13] I. Coanda, A. S. Tikhomirov, G. Trautmann. Irreducibility and smoothness of the moduli space of mathematical 5-instantons over $\mathbb{P}^{3}$. Internat. J. Math. 14 (2003), 1-45.

[14] L. Costa, R. M. Miró-Roig. Monads and regularity of vector bundles on projective varieties. Michigan Math. J. 55 (2007), 417-436.

[15] L. Ein. Generalized null correlation bundles. Nagoya Math. J. 111 (1988), 13-24.

[16] G. Ellingsrud, S. A. Stromme. Stable rank 2 vector bundles on $\mathbb{P}^{3}$ with $c_{1}=0$ and $c_{2}=3$. Math. Ann. 255 (1981), $123-135$.

[17] B. Fantechi, et.al. Fundamental algebraic geometry: Grothendieck's FGA explained (Mathematical surveys and monographs, v. 123). American Mathematical Society, 2005.

[18] R. Hartshorne. Algebraic Geometry. Springer-Verlag, New York, 1977.

[19] R. Hartshorne. Stable vector bundles of rank 2 on $\mathbf{P}^{3}$. Math. Ann. 254 (1978), 229-280.

[20] R. Hartshorne. Stable reflexive sheaves. Math. Ann. 254 (1980), 121-176.

[21] R. Hartshorne, A. P. Rao. Spectra and monads of stable bundles. J. Math. Kyoto Univ., 31 (1991), $789-806$.

[22] R. Hartshorne, I. Sols. Stable rank 2 vector bundles on $\mathbb{P}^{3}$ with $c_{1}=-1, c_{2}=2$. J. Reine Angew. Math. 325 (1981), $145-152$.

[23] G. Horrocks. Vector bundles on the punctured spectrum of a local ring. Proc. London Math. Soc. 14 (1964), 689-713.

[24] D. Huybrechts, M. Lehn. The Geometry of Moduli Spaces of Sheaves, Cambridge Mathematical Library, 2nd edn. Cambridge Univ. Press, Cambridge, 2010.

[25] M. Jardim. Instanton sheaves on complex projective spaces. Collect. Math. 57 (2006), 43-68.

[26] M. Jardim, S. Marchesi, A. Wissdorf. Moduli of autodual instanton bundles. Bol. Soc. Brasil. Mat. (N.S.) 47 (2016), 823-843.

[27] M. Jardim, R. V. Martins. Linear and Steiner bundles on projective varieties. Comm. Algebra 38 (2010), 2249-2270.

[28] M. Jardim, M. Verbitsky. Trihyperkähler reduction and instanton bundles on $\mathbb{C P}^{3}$. Compos. Math. 150 (2014), $1836-1868$.

[29] P. I. Katsylo. Rationality of the moduli variety of mathematical instantons with $c_{2}=5$. In: Lie Groups, Their Discrete Subgroups, and Invariant Theory, 105-111. Advances in Soviet Mathematics, v.8, 1992.

[30] P. I. Katsylo, G. Ottaviani. Regularity of the moduli space of instanton bundles $M I_{\mathbb{P}^{3}}(5)$. Transformation Groups 8 (2003), $147-158$.

[31] H. Lange. Universal families of extensions. J. Algebra 83 (1983), 101-112.

[32] N. Manolache. Rank 2 stable vector bundles on $\mathbb{P}^{3}$ with Chern classes $c_{1}=-1$ and $c_{2}=2$. Rev. Roum. Math. Pures et Appl. XXVI (1981), 1203-1209.

[33] M. Maruyama. Moduli of stable sheaves, I. J. Math. Kyoto Univ. 17 (1977), 91-126.

[34] C. Okonek, M. Schneider, H. Spindler. Vector bundles on complex projective spaces. Birkhauser, Boston, 1980.

[35] A. P. Rao. A family of vector bundles on $\mathbb{P}^{3}$. Space curves (Rocca di Papa, 1985), 208-231, Lecture Notes in Math., 1266, Springer, Berlin, 1987. 
[S] I. R. Shafarevich. Basic Algebraic Geometry 1, 3rd ed., Springer Verlag, 2013.

[36] A. S. Tikhomirov. Moduli of mathematical instanton vector bundles with odd $c_{2}$ on projective space. Izvestiya: Mathematics 76 (2012), 991-1073.

[37] A. S. Tikhomirov. Moduli of mathematical instanton vector bundles with even $c_{2}$ on projective space. Izvestiya: Mathematics 77 (2013), 1195-1223.

[38] A. S. Tikhomirov, S. A. Tikhomirov, D. A. Vassiliev. Construction of stable rank 2 bundles on $\mathbb{P}^{3}$ via symplectic bundles. Sib. Math. J. 60 (2019), 343-358.

iCEx - Ufmg, Departamento de Matematica, Av. Antonio Carlos, 6627, 30123-970 Belo Horizonte MG, Brazil

Email address: charlesalmeida@mat.ufmg.br

imeCC - UniCAmp, Departamento de Matemática, Rua Sérgio Buarque de Holanda, 651, 13083-970 Campinas-SP, BRAZIL

Email address: jardim@ime.unicamp.br

Faculty of Mathematics, National Research University Higher School of Economics, 6 Usacheva Street, 119048 Moscow, Russia

Email address: astikhomirov@mail.ru

Department of Mathematics, Yaroslavl State Pedagogical University named after K.D.Ushinskit, 108 RespublikanSkaya Street, 150000 Yaroslavl, Russia 